\title{
Investigating the Effect of Reduced Serotonin \\ Transporter Expression in a Maternal Immune Activation \\ Model of Neurodevelopmental Disorders
}

by

Alexandra Lister

\begin{abstract}
A Thesis
Submitted to the Victoria University of Wellington

in fulfilment of the requirements for the degree of

Master of Science

In Cell and Molecular Bioscience.
\end{abstract}

Victoria University of Wellington

2021 



\section{Abstract}

Maternal Immune Activation (MIA) during early pregnancy is an established risk factor for the occurrence of neurodevelopmental disorders such as Autism Spectrum Disorder (ASD) and schizophrenia (SCZ) in offspring. Serotonin signalling is also implicated in both ASD and SCZ, in conjunction with a known and extensive influence in neural development. Using a Wistar serotonin transporter (SERT) knockout model to mimic allelic variation in the human serotonin transporter promoter (5-HTTLPR), this research investigates the impact of full or reduced SERT function on the effect of poly I:C-induced MIA in offspring. Experimental design focuses on ultrasonic vocalisation communication in postnatal day (PND) 7 offspring, followed by genetic expression of the Rac1/Kal7/Disc1 signalosome pathway at PND21 previously implicated in SCZ pathology. Results from behavioural analysis of pups indicate a statistically significant increase in calling and call complexity in pups heterozygous for the SERT (SERT HET) compared to wildtype (WT). When separated by sex, this trend remains consistent however only reaches significance in male offspring. Male SERT HET pups also a significant treatment effect in call complexity, and a significant genotype/treatment interaction which suggests an increased susceptibility to MIA-induced behavioural effects. Additionally, poly I:C exposed pups show increased expression of Disc1, supporting evidence that this pathway may be affected in neurodevelopmental disorders. No genotype and sex effects were observed in signalosome expression; however, this study may be too underpowered to detect these effects. These results suggest that differences between sex and SERT genotype in offspring may modulate the behavioural effects of MIA in rodent models of NDD, with more study required to assess these differences in a molecular context. Furthermore, this study aims to address the overall inconsistency and misrepresentation of statistical methods in MIA models by employing MIA validation tests and linear mixed modelling to account for litter variation. In summary, the research presented in this thesis reports initial evidence suggesting SERT genotype may influence the effect of MIA, however further research is necessary to characterise the effect of genotype on MIA challenge during gestation. 
- 4 - 


\section{Acknowledgements}

Firstly, I would like to acknowledge the guidance and support of my Academic Supervisor Dr Darren Day. Through endless unexpected issues and a pandemic no less, your expertise and positivity were essential to my successes and developing from my mistakes. All of our meetings and discussions have had a huge impact on my scientific ability and improved my confidence in my work: I would not be the student I am today without your guidance, and I am thankful and excited to continue learning from you. I would also like to thank my unofficial secondary supervisor Dr Bart Ellenbroek, whose advice on animal research and ethics was invaluable. I could not have completed this project without your assistance, and I am exceedingly grateful.

For training in animal handling, injection lessons, and various other skills, I would like to thank Aimee Culverhouse and Joyce Colussi-Mas. Their aid in caring for the animals in our lab and patience with my endless questions and breeding programmes is much appreciated. Additionally, the uphill battle of linear mixed modelling could not have been won without the patience and enthusiasm of Lisa Woods. The time you set aside to meet me week after week, explaining sequential ANOVAs and developing R code, has improved my research hundred-fold. Maybe if every scientist had a statistician like you to help them, the reproducibility crisis would be less of a crisis and more of a rough day at the office!

Recognition is also due to the past and present members of the Day and Ellenbroek laboratories, who's encouragement and advice not only improved my scientific ability but also strengthened my motivation and love of research. I am endlessly grateful to Jess and Daniel for taking an innocent and inexperienced Alex under their wing, being ready to answer all my questions, and only laughing at me a little bit when I did something embarrassing. Special acknowledgement must of course go to the amazing Bryony Thorne, who has enabled my iced coffee problem and kept me sane for the last two years. I couldn't imagine going into another three years of this chaos without your support! 
On a personal note, I would like to thank all of the Cool Kids Club for being the best friends a girl could ask for. Additionally, for Ashley and Phoebe: I can never express my appreciation and love for you enough, you truly are sisters in every way. Finally, I would like to thank my family for their unwavering love and support. Even at the times when I didn't think I could continue on this path, you never stopped believing in me. I swear in the future I'll dedicate my third Nobel Prize to you and use my endless fortune to buy you some treats. 


\section{Contents}

Abstract

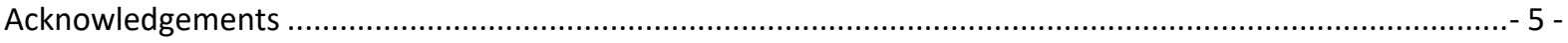

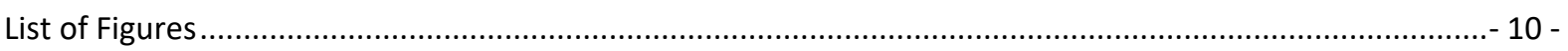

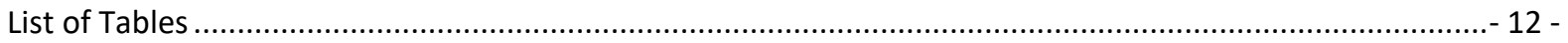

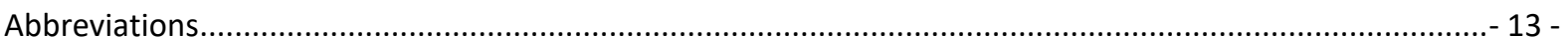

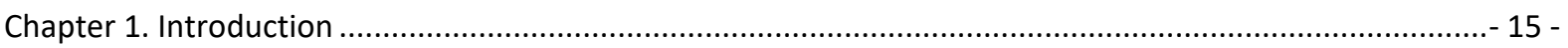

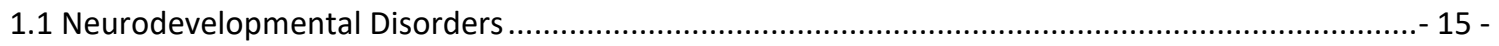

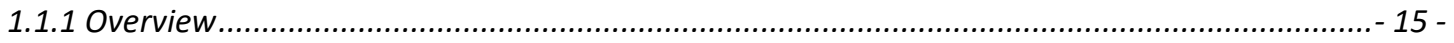

1.1.2 Diagnosis and Psychiatric Comorbidity............................................................................ 16 -

1.1.3 Common Risk Factors ........................................................................................... 17 -

1.1.4 Leading Molecular Models .......................................................................................... 20 -

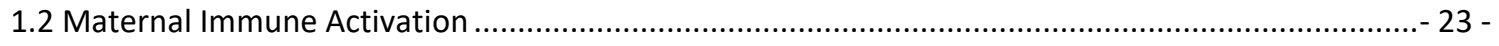

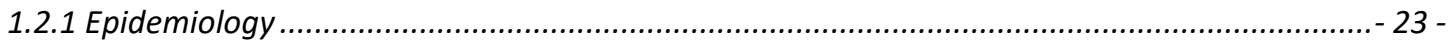

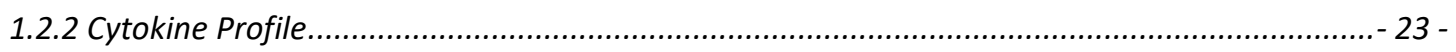

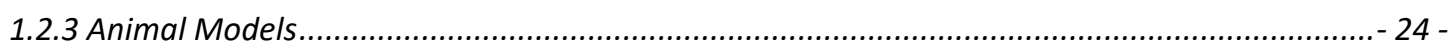

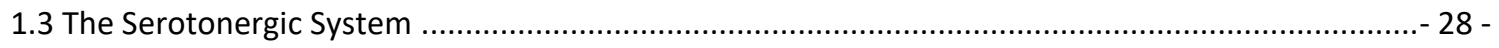

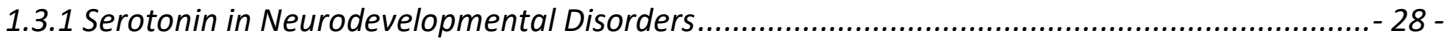

1.3.3 Variable Expression of the Serotonin Transporter ................................................................ 29 -

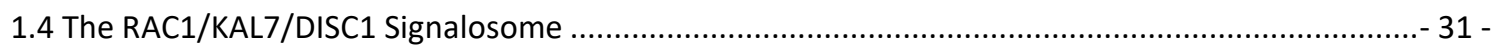

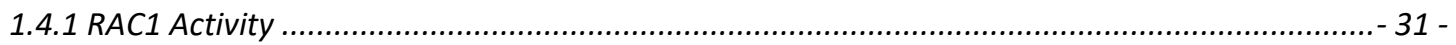

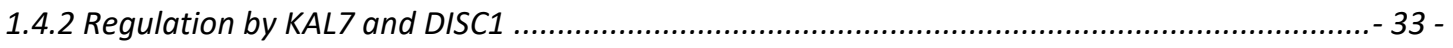

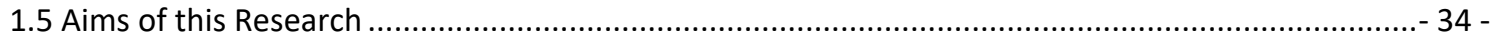

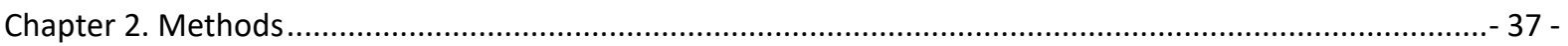

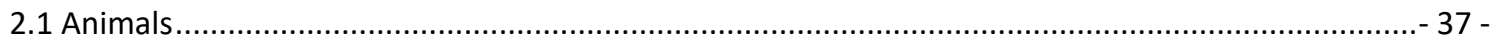

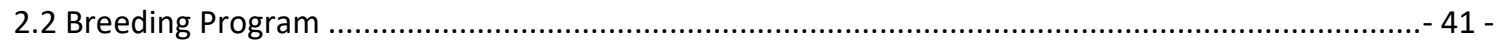

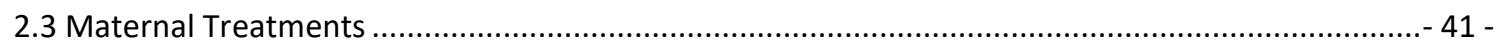

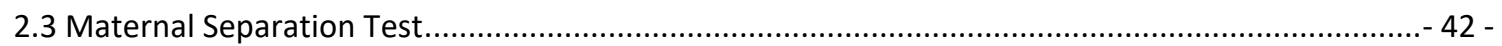

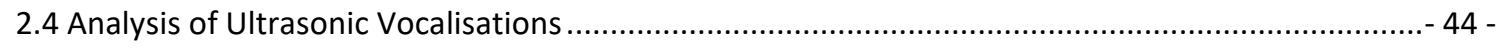

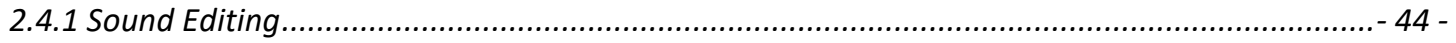

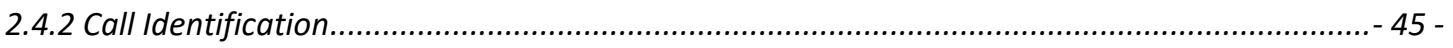

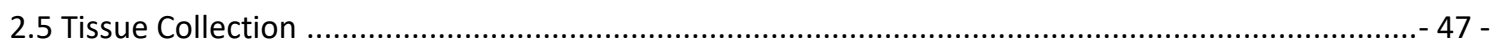




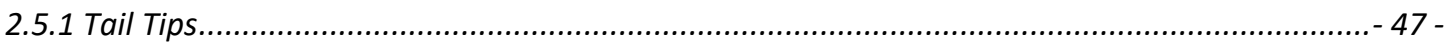

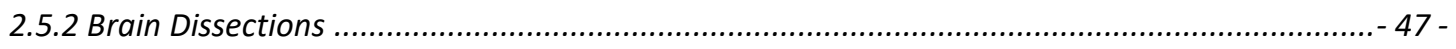

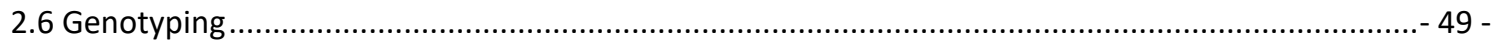

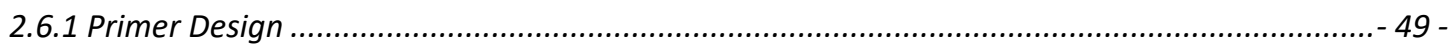

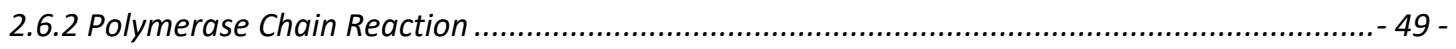

2.6.3 High Resolution Melt Analysis ..................................................................................... 50 -

2.8 Quantitative Reverse Transcription Polymerase Chain Reaction (RT-qPCR)................................ 53 -

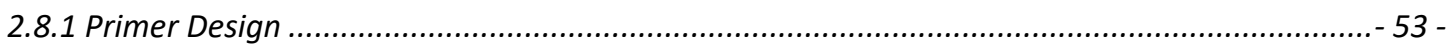

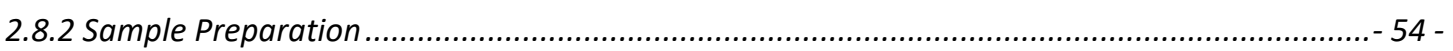

2.8.3 Quantitative Polymerase Chain Reaction (qPCR) ............................................................. 55 -

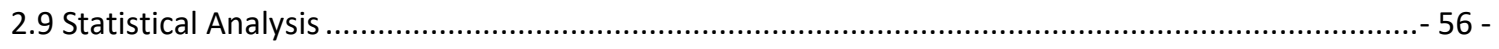

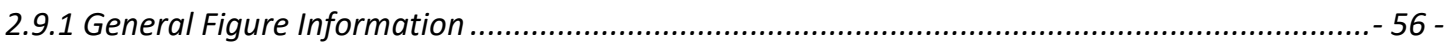

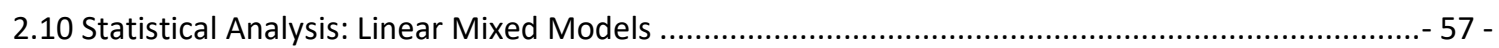

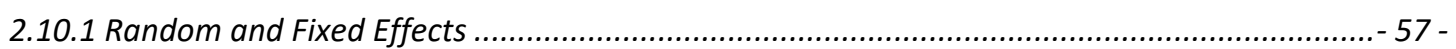

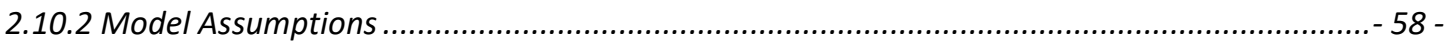

Chapter 3. Maternal Immune Activation Model ............................................................................... 60 -

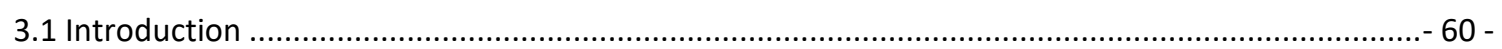

3.1.1. Maternal Immune Response ........................................................................................ 60 -

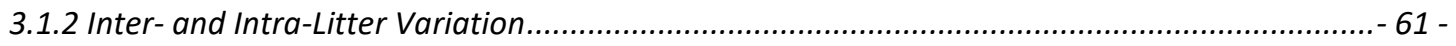

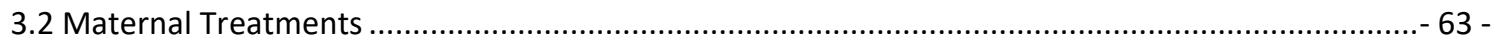

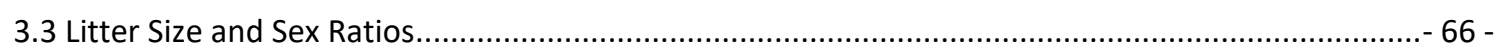

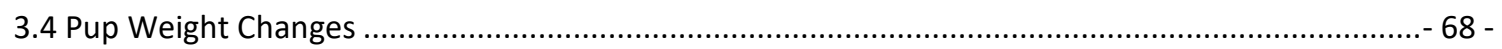

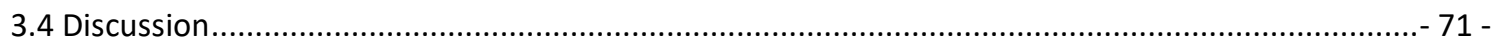

3.4.1 Maternal Immune Response.................................................................................... 71 -

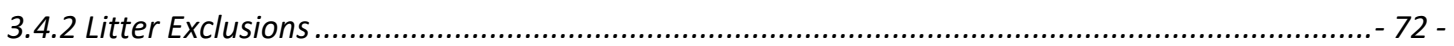

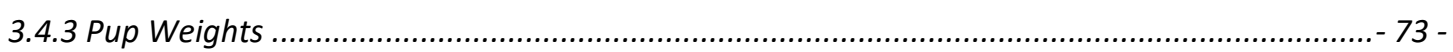

Chapter 4: Behavioural Study; Maternal Separations ........................................................................ 75 -

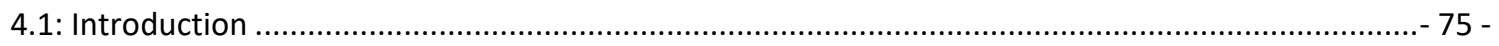

4.1.1 Ultrasonic Vocalisations in Pup Behavioural Research ...................................................... 75 -

4.1.2 USV Measurement and Classification Tools ....................................................................... 76 -

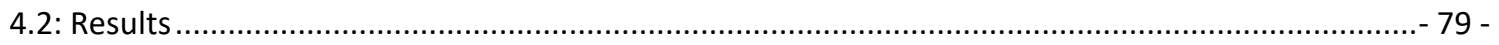

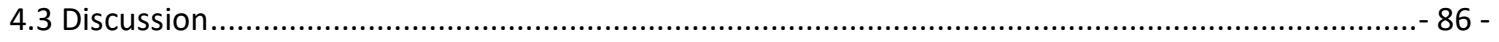

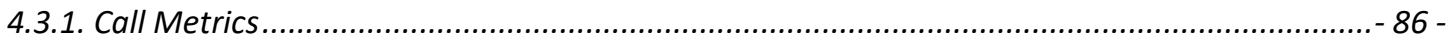

4.3.2 Linear Mixed Models ........................................................................................................ 87 -

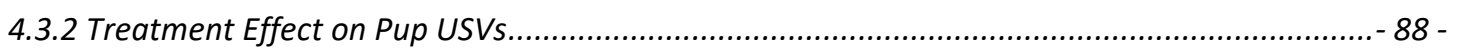

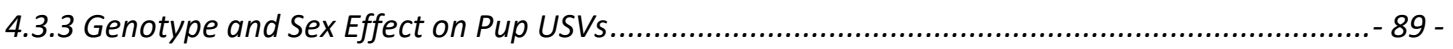




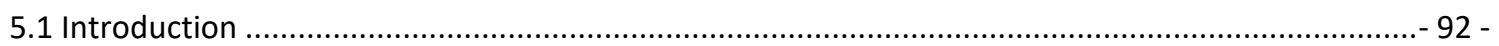

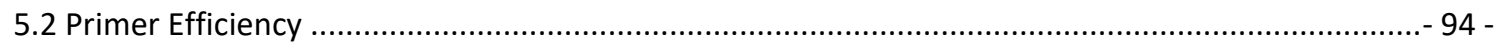

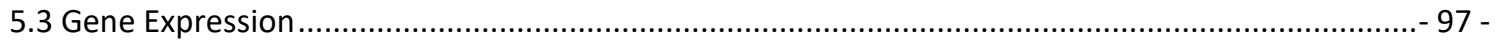

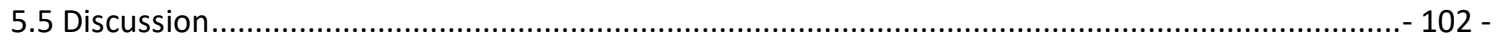

5.5.2 Validity Measures ............................................................................................... 102 -

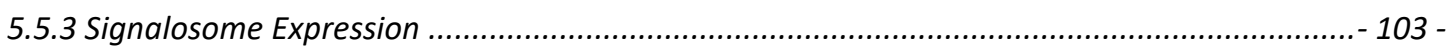

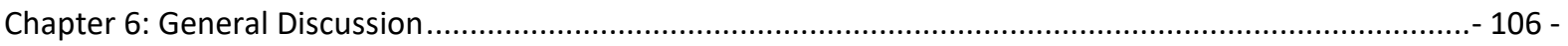

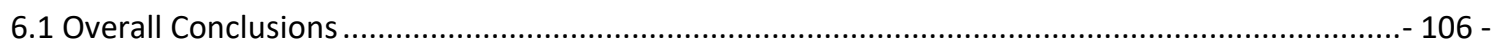

6.1.1 Model Validation and Statistical Analysis ...................................................................... 106 -

6.1.2 Behavioural and Molecular Experiments...................................................................... 108 -

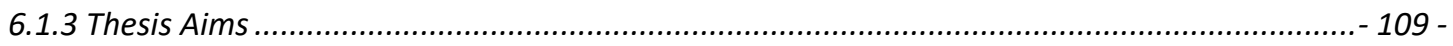

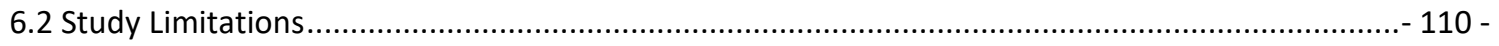

6.2.1 Sample Size and Statistical Power ................................................................................. 110 -

6.2.2 Maternal Immune Response........................................................................................ 110 -

6.2.2 Animal Models in Human Neurological Disorders............................................................. 111 -

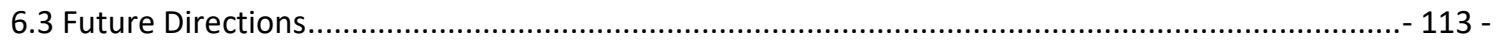

6.3.2 Continued Analysis of the Rac1/Kal7/Disc1 Pathway.................................................... 113 -

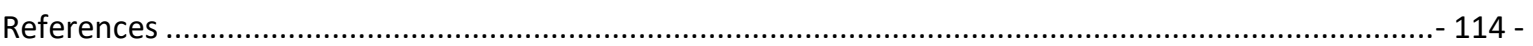

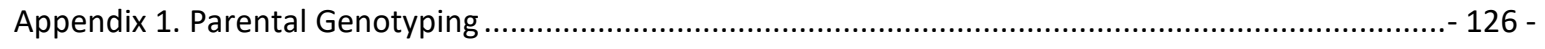

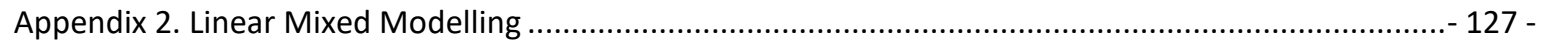

Appendix 3: Non-Significant USV Pairwise Comparisons................................................................ 129 -

Appendix 4: Differentiation of Gene Expression Targets by Sex and Genotype .................................. 131 - 


\section{List of Figures}

Figure 1.1: Gene and Environment Interaction in NDD Causation ....................................................... 18 -

Figure 1.2: Overview of the Maternal Immune Activation Rodent Model ................................................ 25 -

Figure 1.3: Pup Development and MIA Challenge Timeline ............................................................... 26 -

Figure 1.4: Promoter Region Variance in SERT Expression ................................................................. 30 -

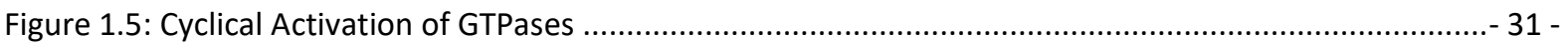

Figure 1.6: Simplified View of the RAC1/KAL7/DISC1 Signalosome ...................................................... 34 -

Figure 1.7: Graphic Representation of Overall Experimental Design .................................................... 36 -

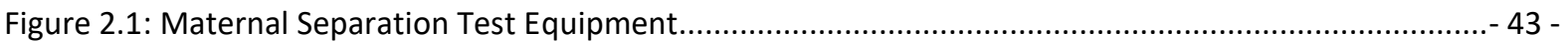

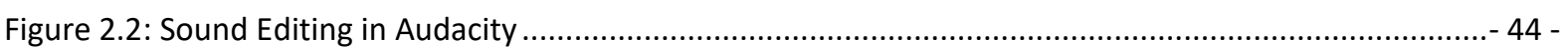

Figure 2.3: DeepSqueak Call Detections Viewed in DeepSqueak Screener ................................................ 46 -

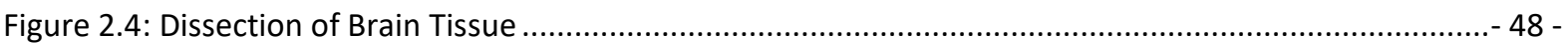

Figure 2.5: Genotyping of Pups Using High Resolution Melt Analysis ....................................................- 52 -

Figure 2.6: Example RStudio Script of Linear Mixed Modelling ............................................................ 59 -

Figure 3.1: Maternal Temperature Change Following Treatment ........................................................... 63 -

Figure 3.2: Maternal Weight Change Following Treatment ............................................................. 65 -

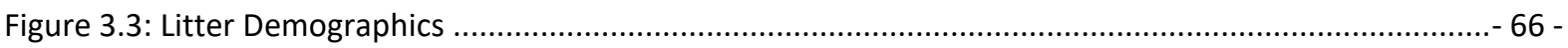

Figure 3.4: Treatment Effect on Pup Weight Gain ........................................................................... 68 -

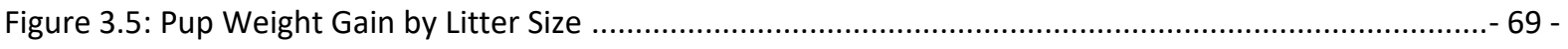

Figure 4.1: Treatment Comparisons Across All Groups ....................................................................... 81 -

Figure 4.2: Significant Sex and Genotype Comparisons Across All Groups .............................................- 82 -

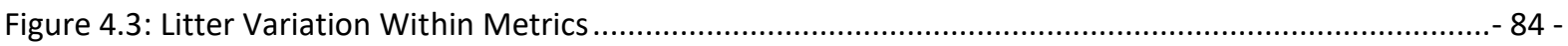


Figure 5.1: Primer Efficiencies for RT-qPCR Primer Sets.

Figure 5.2: Melt Curve Analyses for RT-qPCR Primer Set Products .................................................... 96 -

Figure 5.3: Prox1 Expression Compared Between Researcher ............................................................... 99 -

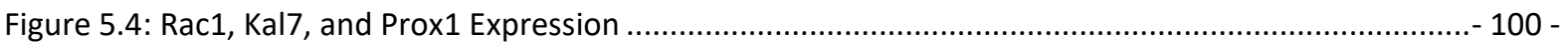

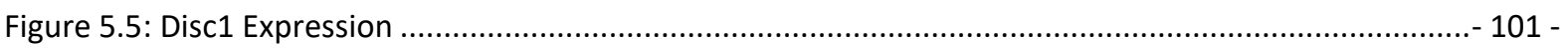

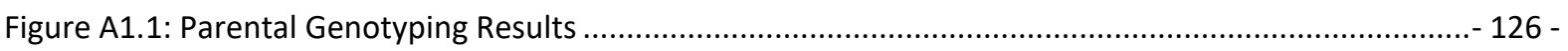

Figure A3.1: Non-Significant Genotype Comparisons ..................................................................... 129 -

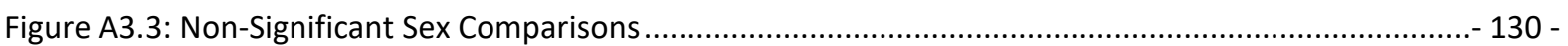

Figure A4.1: Gene Expression of Poly I:C and Saline Exposed Pups Grouped by Sex and Genotype ............- 131 - 


\section{List of Tables}

Table 2.1: Total Breeding Animals and Pups Associated with this Thesis

Table 2.2: Eligible Pups Used or Awaiting Use in RT-qPCR Experiment...... $40-$

Table 2.3 Genotyping Primer Sets $49-$

Table 2.4 RT-qPCR Primer Sets $53-$

Table 4.1: List of Potential and Investigated DeepSqueak Call Metrics...... $78-$

Table 4.2: Overall Effects on USV Metrics $79-$

Table 5.1: Overall Effects on Gene Expression Targets $97-$

Table A2.1: F-statistics for USV Study Using Linear Mixed Modelling ..... $127-$ 


\section{Abbreviations}

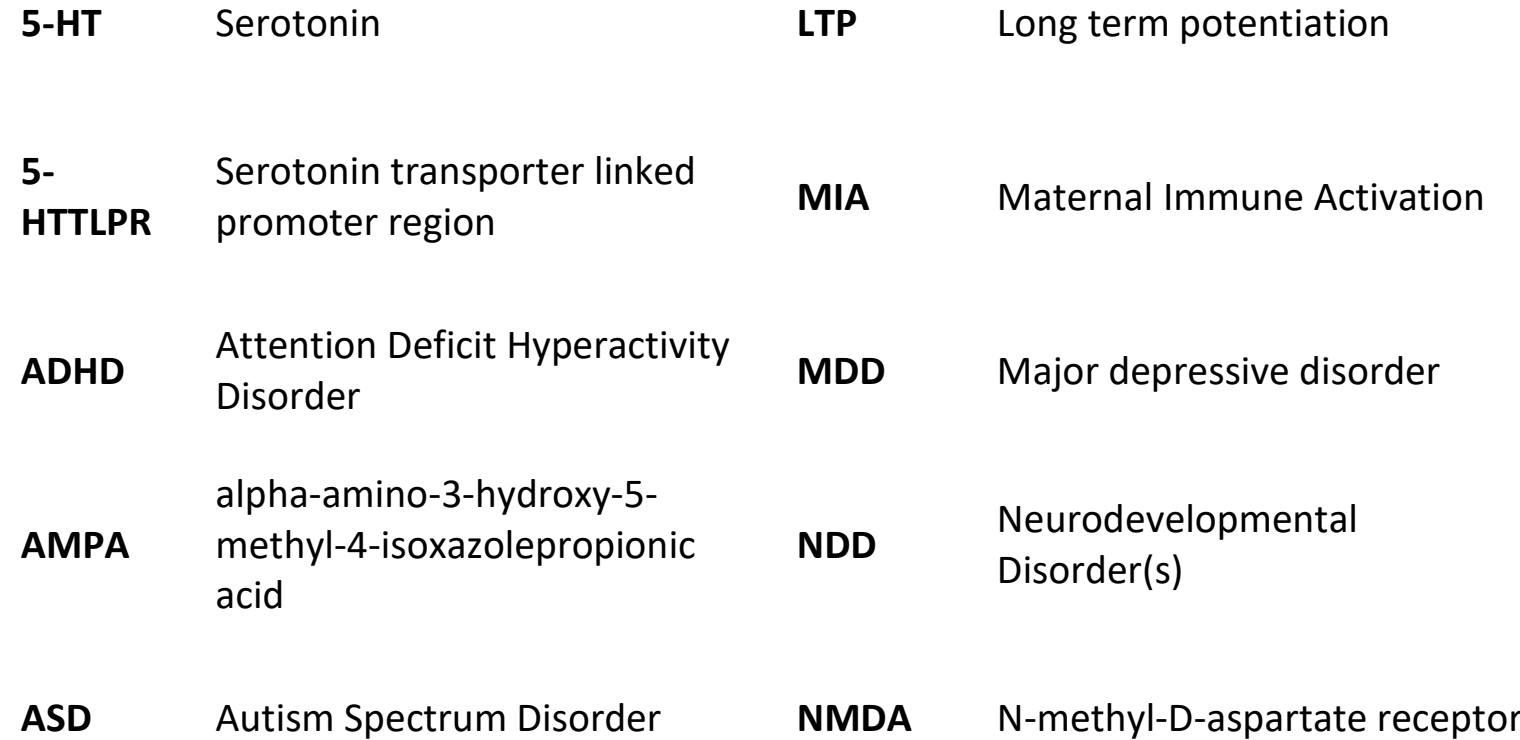

DISC1 Disrupted in Schizophrenia 1 PND Postnatal Day

DSM Diagnostic and Statistical Manual of Mental Disorders

GABA gamma-Aminobutyric acid

GAP GDP Accepting Protein Gapdh $\begin{aligned} & \text { Glyceraldehyde 3-phosphate } \\ & \text { dehydrogenase }\end{aligned}$

qPCR

Gestational day

GDP Guanosine diphosphate

GEF Guanine Exchange Factor
RAC1

Poly I:C Polyinosinic:polycytidylic Acid

Prox1 Prospero homeobox protein 1

Post-synaptic density protein 95

Quantitative Polymerase Chain Reaction

Ras-related C3 botulinum toxin substrate 1

Quantitative Reverse

RT-qPCR Transcription-Polymerase

Chain Reaction

SCZ Schizophrenia
PSD95 


\begin{tabular}{|c|c|c|c|}
\hline GTP & Guanosine triphosphate & SERT & Serotonin Transporter \\
\hline HET & Heterozygous & TLR & Toll-like Receptor \\
\hline HPRT & $\begin{array}{l}\text { Hypoxanthine-guanine } \\
\text { phosphoribosyl transferase } 1\end{array}$ & TNFa & Tumour necrosis factor alpha \\
\hline HRMA & High Resolution Melt Analysis & TSC & Tuberous sclerosis complex \\
\hline IL- & Interleukin & USV & Ultrasonic Vocalisation \\
\hline KAL7 & Kalirin7 & VNTR & $\begin{array}{l}\text { Variable nucleotide tandem } \\
\text { repeat }\end{array}$ \\
\hline LMM & Linear mixed modelling & WT & Wildtype \\
\hline LPS & Lipopolysaccharide & & \\
\hline
\end{tabular}




\section{Chapter 1. Introduction}

\subsection{Neurodevelopmental Disorders}

\subsubsection{Overview}

'Neurodevelopmental Disorders' (NDDs) is an umbrella category for disorders arising during brain development, resulting in a broad range of behavioural, cognitive, and motorrelated phenotypes [1]. Typically, these disorders have overlapping cognitive symptoms resulting in difficulty focusing and learning. Some common NDDs include dyslexia, attention deficit hyperactivity disorder (ADHD), and autism spectrum disorder (ASD). Schizophrenia (SCZ) is also considered to lie on the NDD spectrum; although major symptoms and diagnosis often occur later in life, many biological factors indicate its origins during neurodevelopment [2]. ASD and SCZ are the two main NDDs represented in this thesis research.

Autism Spectrum Disorder covers a range of previously distinct disorders characterised by challenges with social and physical behaviour, alongside altered cognitive ability [3]. Individuals with ASD commonly show a reduced ability to identify and respond to social cues including body language and tonality. Within their own body language, repetitive behaviours are often exhibited in response to strong emotions such as stress or excitement. Challenges with motor skills may also be present. Individuals also often exhibit changes to cognitive ability; some may have difficulty with learning and comprehension, while other may excel [4]. While symptomology may differ between cases, the affected areas of development remain the same. Due to every case consisting of a unique combination of symptoms and symptom severity, ASD has a history of being difficult to diagnose and has changed definitions in the Diagnostic and Statistical Manual of Mental Disorders (DSM) multiple times since the addition of 'Autism' as a form of childhood schizophrenia in the DSM-2 [5]. In the current DSM-5, ASD covers previously distinct diagnoses such as pervasive developmental disorder-not otherwise specified (PDD-NOS) and Asperger Syndrome [3]. 
Schizophrenia is a neuropsychiatric disorder with symptoms categorised as positive, negative, and disorganised [3]. Positive symptoms are often the most recognised as schizophrenic; visual and/or auditory hallucinations, paranoia, and strong delusions. Episodes consisting largely of positive symptoms result in difficulty distinguishing reality from delusion. Negative symptoms present as an unusual loss of behaviour, including not expressing emotion and social withdrawal. Finally, disorganised symptoms include confusing and disorganised thoughts, speech, and abnormal movements. Schizophrenia also often presents with reduced cognitive ability, both related to learning and social processes $[6,7]$. It is important to note that popular media has driven negative stereotypes of schizophrenia by sensationalising positive symptoms as highly dangerous [8]. This stigmatisation likely contributes to the societal cost of schizophrenia, estimated as $7.4 \%$ of total mental health burden [9]; up to $90 \%$ of schizophrenic individuals face homelessness, and have a reduced life expectancy of $10-20$ years [10].

\subsubsection{Diagnosis and Psychiatric Comorbidity}

Diagnosis of NDDs usually occurs at disorder-specific ages when symptoms begin to present or become noticeable. ASD is often diagnosed in early childhood, when social and learning difficulties become apparent as children enter early schooling; alternatively, SCZ is usually diagnosed in early adulthood when psychosis symptoms start to present [11]. With both disorders, symptoms persist throughout life once presented and may be more easily managed with medication but not permanently altered.

Interestingly, diagnosis trends in ASD and SCZ are very similar. For example, both disorders are reported to have an increasing diagnosis rate worldwide $[12,13]$. Both disorders are also more likely to be diagnosed in males than females; for ASD males are 3-4x more likely, and SCZ males are $1.5 x$ more likely $[14,15]$. Interestingly, age of diagnosis reverses this trend in SCZ, where females are more likely to be diagnosed later in life $[16,17]$. These sex differences may be influenced by unconscious biases from the diagnostic perspective, originating from different societal expectations of behaviour between sexes [18]. Current evidence also suggests this may be driven by biological differences in sex, with many disorder- 
linked genes being found on the $X$ chromosome $[19,20]$. This could result in potential silencing/compensation of deleterious alleles by the other $\mathrm{X}$ chromosome in heterozygous biological females, resulting in a lower prevalence. The 'female protective effect' described in many NDDs as the phenomenon where females often carry more deleterious mutations than affected males [21]. It is also suggested that sex hormones such as oxytocin may have a protective role in the brain of female subjects [22].

Another diagnostic trend conserved between ASD and SCZ are their comorbidities. This is particularly important as common comorbidities may provide insight into affected systems and inform future research direction for a disorder. An interesting point to consider is that while NDDs have distinct diagnoses, it is possible for an individual to present symptoms for more than one; the most common overlap is between ADHD and ASD symptomology, with $22-83 \%$ of ASD cases being co-diagnosed [23]. ASD and SCZ may also be co-diagnosed, with recent research estimating a 3.5x higher diagnosis in ASD than non-ASD individuals [24]. Many other psychiatric disorders are co-diagnosed with ASD and SCZ particularly anxiety and depressive disorders. The negative symptoms of schizophrenia align with those of major depressive disorder (MDD), making their association unsurprising: recent studies have suggested clinical MDD episodes in up to $80 \%$ of first psychosis cases [25], with an overall rate of comorbidity at approximately $30 \%$ [26]. Individuals with schizophrenia also present anxiety disorder symptoms, with social anxiety disorder being most commonly co-diagnosed [27]. Anxiety and depressive disorders also show a high prevalence in ASD, with higher intellectual and cognitive functioning correlated with increased incidence of both [28, 29].

\subsubsection{Common Risk Factors}

While no specific cause of schizophrenia or autism spectrum disorder has been identified, there is consensus within the literature that both genetic and environmental factors play a role in disorder development. This is often presented as a form of the 'multiple hit hypothesis', where genetic priming or early environmental shock acts to reduce the ability of the individual to tolerate later environmental stresses [30, 31]. This may explain the high variation in symptomology and affected pathways in ASD; different combinations of genetic 
and environmental risk factors may be responsible for the disorder across individuals, resulting in slightly varied biological changes and therefore varied phenotypes. This model could also allow for late-stage onset of schizophrenia symptoms, after life stressors and biological changes in adolescence act as a 'second hit' from disorder priming during development (Figure 1).

\section{Figure 1.1: Gene and Environment Interaction in NDD Causation}

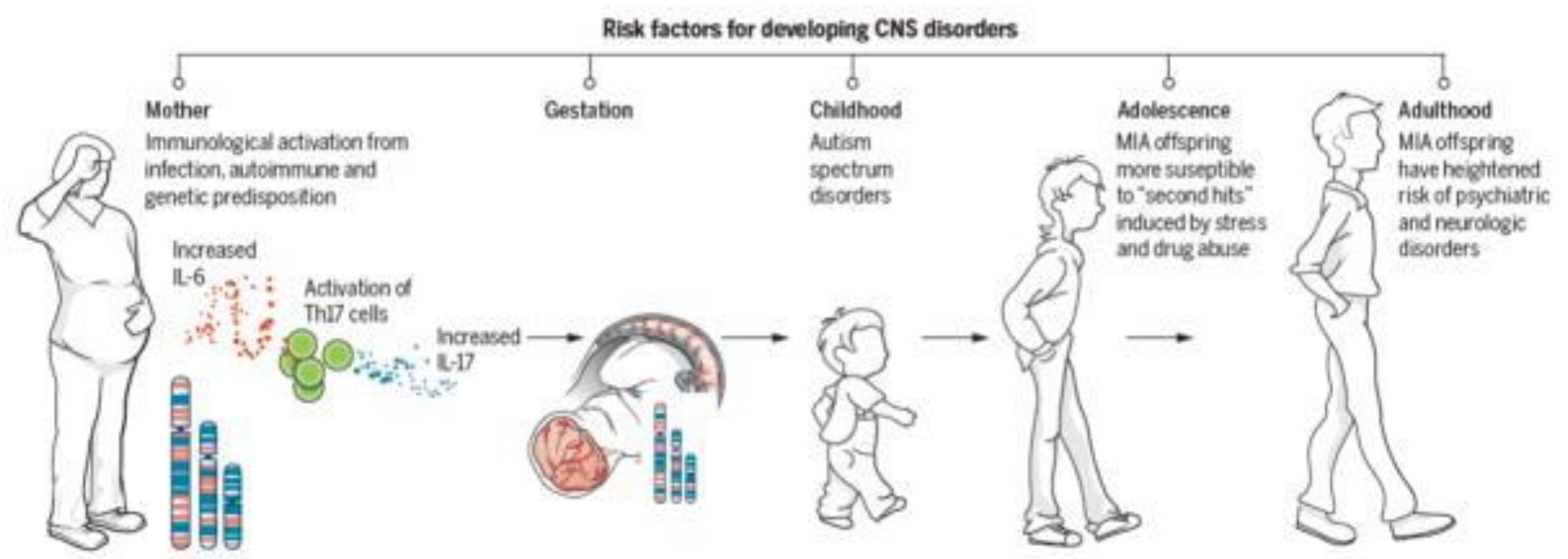

Figure 1.1: A timeline of genetic and environmental influences that may contribute to risk of neurodevelopmental and neuropsychiatric disorders in offspring. Genetic priming can result in susceptibility to environmental 'second hits', either occurring prenatally such as maternal immune activation (MIA) or postnatally such as stress in adolescence. Figure sourced from Estes et al. 2016 (Reference 31). Reprinted in this thesis with permission from AAAS.

There is a large genetic risk component to ASD and SCZ, however estimation of this component has varied widely. Early twin studies assessing the heritability of ASD supported a heritability of approximately $90 \%$, while later studies have significantly lower estimates near $30-50 \%[32,33]$. This difference may be impacted by a) changes to diagnosis of ASD, and $b$ ) new knowledge of epigenetic and environmental differences between monozygotic twins, such as epigenetic programming and $X$ chromosome silencing in females [34]. A recent analysis of the Danish Twin Register estimates the heritability of SCZ at a high rate of 79\%, however note that non-genetic risk factors will also play a large role in disease development [35]. 
Hundreds of genes and allelic variants have been associated with ASD and SCZ. Most are novel polymorphisms identified through Genome-Wide Association Studies (GWAS) or are identified as candidate genes based on hypothesised causes or mechanisms [36, 37]. Specific genetic risks identified for ASD often stem from their association with commonly comorbid genetic disorders; For example, Fragile $\mathrm{X}$ Syndrome and Tuberous Sclerosis Complex (TSC). The large insertion in the CGG repeat region of the FMR1 gene on the $\mathrm{X}$ chromosome commonly responsible for Fragile X Syndrome and has been significantly linked to ASD in many studies $[38,39]$, as have the mutations in the TCS1 and TSC2 genes causative of TSC [40]. Meta-analyses have found that many of the genes associated with ASD are ontologically related to synaptic transmission and neuronal components [41]. Similarity in genetic risk with comorbidities is also true in schizophrenia, with co-occurring conditions such as Type 2 Diabetes sharing common genetic risk factors $[42,43]$. The genetic risks for ASD and SCZ are also often shared between themselves; for example, neurexins and neuroligins are extensively implicated in both disorders [44]. One recent study estimated the genetic correlation between ASD and SCZ at 23\% [45].

Many environmental factors pose a risk in the development of NDDs. One of the most well-supported environmental risks of ASD and SCZ in offspring is maternal immune activation (MIA) during early pregnancy; this will be expanded on in Chapter 1.2. Increased parental age and exposure to excess of sex hormones have also been identified as potential risks in ASD [46]. In SCZ, environmental risk factors commonly refer to traumatic experiences during youth which trigger biological changes and progress disorder phenotypes [47]. Additionally, gut microbiota colonies have been increasingly studied in relation to ASD and SCZ pathology. These colonies can affect gene expression and other processes in the brain through the release of metabolites and other bioactive compounds, which activate signalling through the vagus and enteric nerves [48]. Studies have shown that microbiota balance is usually altered in ASD individuals compared to neurotypical individuals, with phyla such as Bacteroidetes and Firmicutes commonly identified $[49,50]$. Reported changes in these phyla are not consistent however [51]. Findings are also notably inconsistent in SCZ research $[52,53]$. Recent projects such as the Genome, Environment, Microbiome and Metabolome in Autism (GEMMA) study 
aim to combine microbiome information with immune and genetic factors from ASD individuals to further clarify the role of the microbiome [54].

As a final note, concern in the public also exists surrounding the claim that vaccines are an environmental factor that may cause ASD. Modern distrust of vaccines rose as a concern over the Measles-Mumps-Rubella (MMR) vaccine led by a now-retracted paper by Andrew Wakefield [55]. Public concern has now spread to other vaccines. As the timing of the MMR and other childhood vaccines coincides with the common diagnosis age for ASD, anecdotal cases exist where causation is inferred but not supported scientifically. This anecdotal evidence, along with scientific mistrust has increased vaccine scepticism [56]. No strong biological evidence exists to support a link between ASD and vaccines, and consensus in the literature supports the opposite; vaccines are not an environmental risk factor for ASD [57].

\subsubsection{Leading Molecular Models}

Most ASD and SCZ research aligns with one of three leading molecular models of NDDs; Immune dysregulation, altered synaptic signalling, and altered brain connectivity. Each model is well-supported to be involved in the pathology of ASD and SCZ and overlap regularly. It is unclear however whether the changes in each model are causative of NDDs, or downstream effects of other pathways being altered. This makes development of therapeutic targets and strategies an elusive goal. Given the understanding that a range of genetic and environmental factors are involved in NDD development, it likely that a range of causative changes exist in subgroups of ASD and SCZ cases and trigger downstream dysregulation across the molecular models.

The immune hypothesis of NDDs primarily focuses on the role of chronic neuroinflammation in ASD and SCZ individuals. Autoimmunity is one mechanism suggested to be involved; for example, being triggered in ASD offspring by transferred maternal antibodies against foetal neural components $[58,59]$. Immune research often focuses on microglia, which are the primary immune cells in the brain and one of the earliest cell types to differentiate during development [60]. Chronic activation of microglia has been implicated 
in ASD pathology repeatedly, where a range of immune factors such as proinflammatory cytokines are regularly released [61]. High levels of cytokines such as interleukin-6 (IL-6) and tumour necrosis factor alpha (TNF $\alpha$ ) have been shown in ASD and SCZ; animal models of overexpressed IL-6 alone have even been reported to present ASD-like phenotypes [62-64]. Particularly, childhood trauma in SCZ has been linked to an increase in these and other proinflammatory cytokines [65]. Over-activation of microglia may be influenced by many pathways including maternal immune activation [66] or the gut microbiome [67]. Another potential activator of microglia is overactive mast cells triggered by high stress; a process also implicated in anxiety disorders, providing another potential link between these disorders [68]. Interestingly, a primary effect of microglial activation is synaptic pruning [69]. This directly allows immune dysregulation to influence signalling and connectivity mechanisms.

Signalling imbalance is another common molecular mechanism of ASD and SCZ pathology [70]. Elements of the dopamine and serotonergic signalling systems have been implicated in NDDs, alongside comorbidities such as anxiety and depressive disorders [71-73]. Another common comorbidity of ASD and SCZ is epilepsy [74, 75] . A primary system dysregulated in epilepsy and further associated with NDDs is the excitatory/inhibitory signalling balance [76]. Glutamate and Gamma Aminobutyric Acid (GABA) signalling, often referred to as excitatory and inhibitory signalling respectively, are primary regulators of synaptic strengthening and plasticity [77]. Increased glutamatergic signalling promotes growth and strengthening of the excitatory synapse, through prolonged activation of AMPA and NMDA receptors [78]. This activates long term potentiation processes such as actin cytoskeleton remodelling through NDD associated proteins such as RAC1 [79]. Additional evidence exists toward dysregulated glutamate signalling, such as the FMR1 mutation associated with ASD and Fragile $X$ syndrome which has been shown to reduce glutamate signalling and synaptic plasticity [80].

In contrast, a major role of GABAergic signalling is to prevent synaptic strengthening through hyperpolarising post-synaptic neurons [81]. One recent study associated loss of function variants of the GABA transporter gene SLC6A1 with increased SCZ and bipolar disorder occurrence [82]. Additionally, the neuroligin genes repeatedly associated with NDDs are linked to the formation of glutamatergic and GABAergic synapses [83]. Changes to gene 
expression of NLGN2 is associated with altered GABAergic synapse formation and signalling balance [84]. Importantly, developmental GABA signalling has been shown to have an excitatory effect; altered GABA signalling during development may contribute to the altered excitatory/inhibitory balance later in life $[81,85]$. The excitatory/inhibitory balance allows synaptic alteration in response to repeated stimulus and weakening in its absence, an underlying mechanism in learning and memory. Social deficits in ASD and SCZ are partially hypothesised to be a result of altered signalling balance brain regions such as the hippocampus, involved in emotion processing and memory [86]. This is one of the primary brain regions focused on in NDD research $[87,88]$, alongside regions primarily associated with motor control and higher thought processes such as the cerebellum and frontal cortex [89].

It is not only synaptic signalling dysregulated in NDDs, but synaptic connectivity as a whole. Reduced connectivity between brain regions as reported in ASD alters the processing of stimuli, potentially contributing to ASD associated difficulties such as identifying complex social behaviours [90]. Lack of connectivity to the ventral hippocampus by excitotoxic shock in rats has been shown to present SCZ relevant behaviours [91]. Altered connectivity within brain regions may also pose an increased risk of NDDs, with sensory-associated regions typically showing decreased connectivity and higher thought-associated regions showing increased connectivity [92]. Kozhemiako recently demonstrated that local connectivity within limbic regions is increased in ASD individuals, and this effect may be stronger in affected females [93]. Recently, mineralocorticoid receptor NR3C2 has been significantly associated with ASD; knockouts in mice have been associated with loss of differential development of the hippocampal CA2 region, and reduction in connectivity between the hippocampus and the hypothalamus $[94,95]$. Genes associated with axon guidance and synaptic formation during development have also been showed to be altered in NDDs. For example, TCS1/TCS2 mutations causative of the TSC disorder play an important role in regulating the mTOR signalling pathway, which when dysregulated can lead to atypical axon growth and impact neural connectivity $[96,97]$. 


\subsection{Maternal Immune Activation}

\subsubsection{Epidemiology}

Maternal immune activation (MIA) is a phenomenon that has been strongly associated with increased risk of disorders such as ASD and SCZ in offspring. A severe immune response during the first trimester of pregnancy may lead to inflammatory immune factors altering the placenta or crossing the placenta and affecting the developing foetus [98, 99]. These are thought to interfere with normal neural development, particularly as neurogenesis and early cell patterning are occurring during this timeframe [100].

Epidemiological studies assessing large birth cohorts have been instrumental in implicating immune response in the development of NDDs. These studies have linked a wide range of diseases to the development of NDDs, including viral and bacterial infections [31]. Both maternal toxoplasmosis and rubella have been associated with an increase in the incidence of schizophrenia in offspring $[101,102]$. Maternal influenza has also been repeatedly linked to NDDs [103-105]. Epidemiological studies have shown it must be a strong immune response - such as high fever or highly increased proinflammatory cytokine levels to result in the changes that can result in NDD development. Studies which do not differentiate between weak and strong immune response have historically produced mixed results, while those that do consistently show a correlation between MIA and increased NDDs in offspring $[106,107]$. Studies assessing increased maternal immune factors alone also find an association with risk of NDDs, suggesting it is the immune response itself, rather than the specific infection, that is important for NDD development [108].

\subsubsection{Cytokine Profile}

The primary driver of NDD development is the increase of a range of proinflammatory cytokines and chemokines triggered during the immune response [31]. Pro-inflammatory cytokines are largely expressed by activated macrophages, and act to initiate or potentiate downstream inflammatory responses to infection such as swelling and fever [109]. During 
MIA, many cytokines have been reported as increased in the bloodstream: these include IL $1 a,-1 b,-4,-5,-6,-17 a$, TNF $\alpha$, and interferon- $\gamma$ [110-112]. These are also reflected in animal models, where similar cytokine profiles are also shown in the amniotic fluid and foetal microenvironment $[113,114]$. IL-6 is the most prominently researched of all MIA-associated cytokines. Studies have shown maternal IL-6 alters hormone production within the placenta and can pass through the placenta to the developing foetus [98, 99, 115]. Once present, IL-6 promotes a long period of inflammation through positive feedback and is further shown to be chronically highly expressed as discussed in Section 1.1.4 [116]. Interestingly, knockout models of the IL-6 receptor in the placenta show no behavioural changes in offspring associated with increased maternal IL-6, suggesting the placenta may be an effective target in preventing the effects of MIA [117].

During the ongoing Covid-19 pandemic concern has arisen surrounding the effect Covid19 may have on NDD prevalence, and the potential high risk to individuals with NDDs that may become infected. SARS-COV-2 has been shown to produce a 'cytokine storm'; a large increase in a range of immune factors, including pro-inflammatory cytokines associated with MIA such as IL-6 and TNF $\alpha$ [118]. It is likely therefore that an individual who becomes infected with SARS-COV-2 during early pregnancy will subsequently have a higher risk of ASD and SCZ in their children. Additionally, ASD and SCZ have both shown evidence of altered immune activity. This, alongside comorbidity of risk factors such as diabetes, may increase the likelihood of NDD-affected individuals presenting more severe symptoms such as Acute Respiratory Distress Syndrome [119].

\subsubsection{Animal Models}

In the current literature, common agents used to simulate MIA are lipopolysaccharide (LPS) and polyinosinic:polycytidylic acid (poly I:C). These trigger immune responses when recognised by the immune system without causing actual disease [114]. LPS is a naturally occurring protein found as a major protective component in the outer cell membrane of gram-negative bacteria. The human innate immune system is able to recognise LPS as a pathogen-associated molecular pattern (PAMP) through binding of Toll-Like Receptor 4 
(TLR4), and activates downstream immune responses such as fever [120]. LPS is therefore commonly used to mimic the effects of bacterial infection, without true bacterial infection. Poly I:C is alternatively a viral mimetic, consisting of short double-stranded RNA fragments. These mimic the structure of viral DNA and is a recognised PAMP when presented extracellularly to Toll-Like Receptor 3 (TLR3). Downstream cytokine increases including IL-6 lead to inflammatory response such as fever, similarly to LPS [121]. Administration of either reagent to a pregnant animal (most commonly rodents) can be used to model immune activation effects on the placenta and foetus (Figure 1.2) [122].

\section{Figure 1.2: Overview of the Maternal Immune Activation Rodent Model}

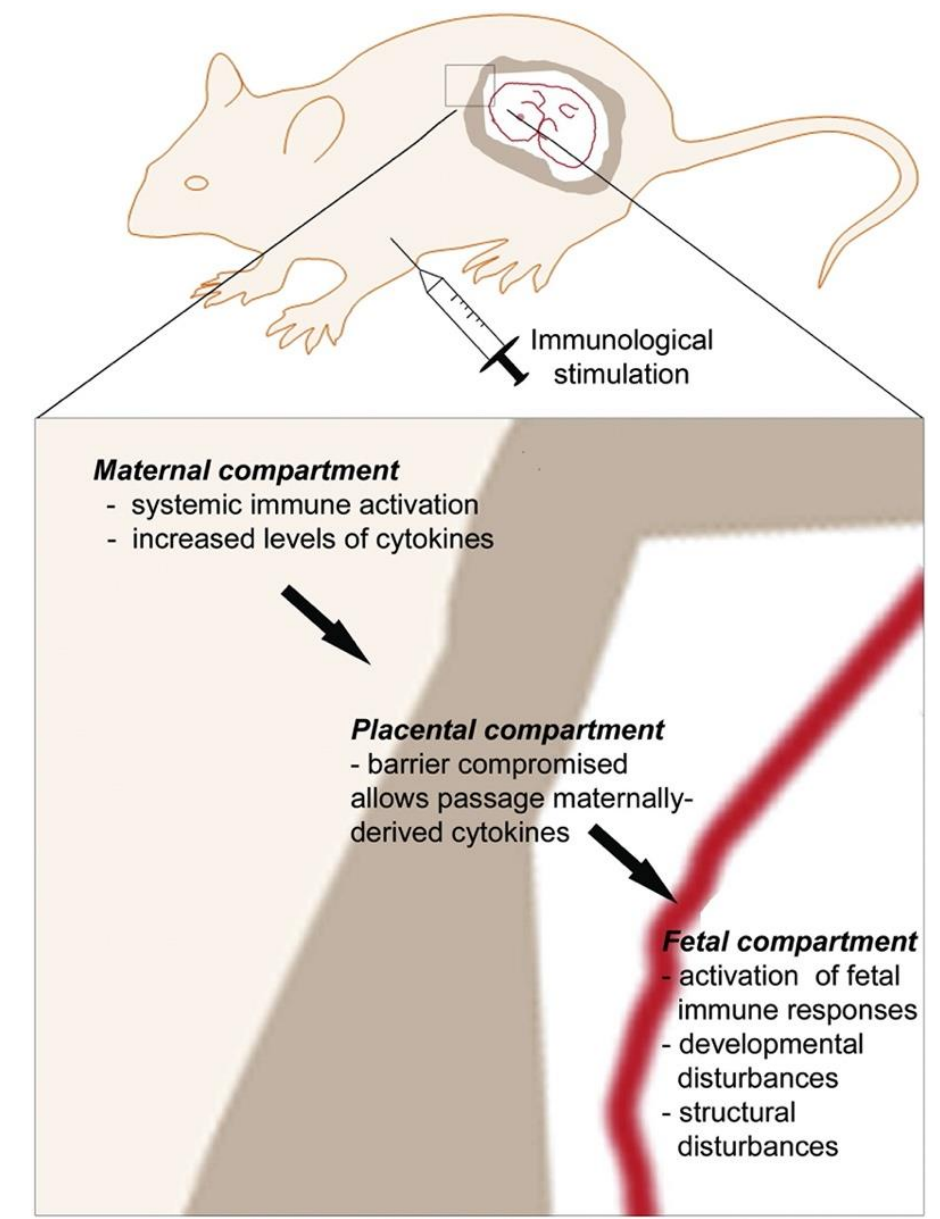

Figure 1.2: Maternal immune activation in rodents. Administration of an immune stimulant such as poly I:C or LPS increases cytokine levels in the maternal system, which alter placental activity and allow foetal exposure to immune factors during development. Figure sourced from Reisinger et al. 2015 (Reference 122), with permission to reprint in this thesis under the Creative Commons CC BY-NC-ND 4.0 licence. 


\section{Figure 1.3: Pup Development and MIA Challenge Timeline}

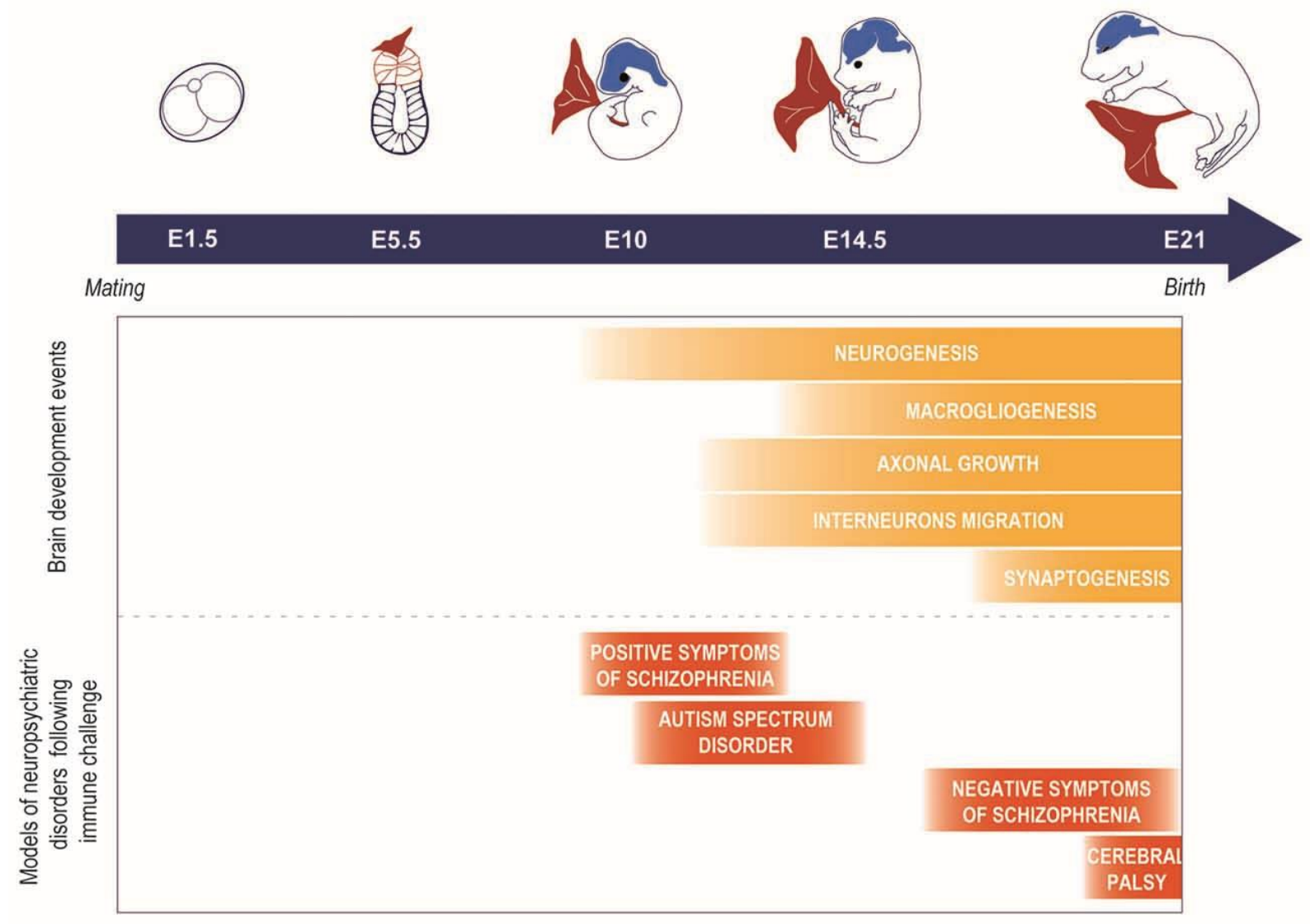

Figure 1.3: A comparison of pup neural development and the common timing of MIA challenge in research. Timing is stated in embryonic days (E), equivalent to gestational day (GD) used elsewhere. The beginning of neurogenesis occurs at approximately E10, alongside the common experimental window used for SCZ and ASD research. Figure sourced from Lombardo et al. 2018 (Reference 127), with permission to reprint in this thesis under the Creative Commons CC BY-NC-ND 4.0 license.

Recent MIA research typically uses an injection of LPS, Poly I:C, or a treatment schedule with multiple injections to represent 'multiple hit' models $[123,124]$. These injections are not consistent between studies; injection method, dosage, and timing all vary largely, making it difficult to compare conflicting results. For example, comparison of behavioural studies with different time points show variability between and within groups [125]. Patterson reports a summary of a wide range of MIA studies, with poly I:C dosage administered intraperitoneally, 
subcutaneously, or intravenously across one or multiple time points at concentrations between 4 and $20 \mathrm{mg} / \mathrm{kg}$ [103]. Large dosage variance may be resulting in immune activation differences between studies that are not fully accounted for, and masking other variance within the models of biological significance [124]. Timing of Poly I:C or LPS administration is also highly varied and may have important outcomes on the health and biological relevance of the model, depending on the phase of brain development $[126,127]$. MIA studies usually occur between GD9-17 (Figure 1.3) to target similar neural developmental processes to those occurring during the first trimester of human pregnancy [31]. Administration of LPS or Poly $\mathrm{I}: \mathrm{C}$ at the earlier end of this range (before GD10) may damage to the placenta however, leading to brain hypoxia-driven effects and litter loss [116]. 


\subsection{The Serotonergic System}

\subsubsection{Serotonin in Neurodevelopmental Disorders}

Serotonin $(5-\mathrm{HT})$ one of the most widespread neurotransmitters in the brain, innervating almost every brain region [128] with serotonergic neurons present as early as 5 weeks into gestation [129]. At least 14 serotonin receptor subtypes have been characterised, suggesting a range of potential downstream targets [130]. The proposed roles of 5-HT throughout life reflect this extensive presence, with implications in processes ranging from neurogenesis, synaptogenesis, and neural morphology in foetal development to regulation of adult neurogenesis, synaptic plasticity and epigenetic modification in the adult brain [131]. These implicated roles of serotonergic signalling contribute to overarching modulation of processes such as higher cognition and emotional regulation, key areas of dysregulation in many neurodevelopmental and neuropsychiatric disorders [131].

There are multiple paths of evidence which implicate altered 5-HT signalling in the pathology of NDDs. Firstly, the influential role of 5-HT signalling in neurodevelopment is well established. Neural morphology is highly regulated by early serotonergic signalling, clearly suggesting a role in the altered brain connectivity observed in ASD and SCZ affected individuals [132]. Additionally, the multiple regulatory pathways of 5-HT in synaptic stability provides strong evidence to suggest a role in the synaptic dysregulation observed in ASD [131]. Altered serotonergic signalling has also been associated with common comorbidities of ASD and SCZ, such as anxiety and depressive disorders [133]. Serotonin has been associated with the development and function of key systems in stress response that are dysregulated in anxiety disorders, such as the hypothalamic-pituitary-adrenal axis (HPA) [134]. Additionally, one of the primary treatment targets for MDD and other depressive disorders is the serotonin transporter (SERT). The serotonin transporter is essential for reuptake of 5-HT following synaptic signalling, for either recycling or degradation by monoamine oxidase [135]. Selective Serotonin Reuptake Inhibitors (SSRIs) inhibit the activity of the serotonin transporter, likely resulting in the increased activity of serotonin in the synapse by preventing reuptake to the presynaptic terminal [136]. Finally, serotoninergic signalling acts in translating signals across 
the gut/brain axis; this may directly impact the role gut microbiota is hypothesised to play in disorders such as ASD [137].

The serotonergic system has also been directly associated with NDDs in multiple contexts. Meta-analysis of genes implicated in SCZ reveal associations with many processes in the serotonin system, including synthesis, transport, and intracellular signalling of 5-HT [138]. Serotonin has been hypothesised to play a role in both the negative and positive symptomology of schizophrenia; the association of 5-HT with depressive and anhedonic behaviour has been long established, and the role of $5-\mathrm{HT}$ and $5-\mathrm{HT}$ receptor targeting medications in hallucinations and psychosis suggests an involvement in delusion symptoms $[139,140]$. It has long been recognised that ASD individuals are also affected by altered serotonergic signalling. Altered levels of 5-HT are reported in both the peripheral and central nervous systems; commonly, hyperserotonaemia is observed in peripheral blood while hypoor hyper- serotonaemia is observed within the brain [141, 142]. Low expression of the serotonin transporter (SERT) is observed in adults with ASD, suggesting altered neural serotonin signalling during adulthood [143].

\subsubsection{Variable Expression of the Serotonin Transporter}

Transcription and subsequent expression of SERT in humans is known to be influenced by variation in the promoter region of the SERT gene (Figure 1.4) [144]. The promoter region associated with the SERT encoding gene, SLC6A4, contains the serotonin transporter linked polymorphic region (5-HTTLPR). Within this region a variable number tandem repeat (VNTR) exists, which is present in humans either as the short (VNTR-S) or long (VNTR-L) allelic variant. Both alleles are well established, with early studies suggesting Caucasian and Asian populations having the highest incidence of VNTR-S alleles [145]. Individuals carrying the VNTR-S allele have been previously reported to express less SERT compared to those carrying the VNTR-L, and have historically been associated with a higher risk of developing anxiety and depressive disorders [146]. The relationship between allelic variants of the 5-HTTLPR and mental disorders such as MDD is currently unclear, as recent high-powered studies have refuted previous findings and suggested no association exists [147]. To clarify the role of 5- 
HTTLPR variants in MDD and other associated disorders, it has been suggested that a wider range of studies should be employed to gain a stronger understanding of how environmental factors may contribute to the effects of varied SERT expression [148].

\section{Figure 1.4: Promoter Region Variance in SERT Expression}

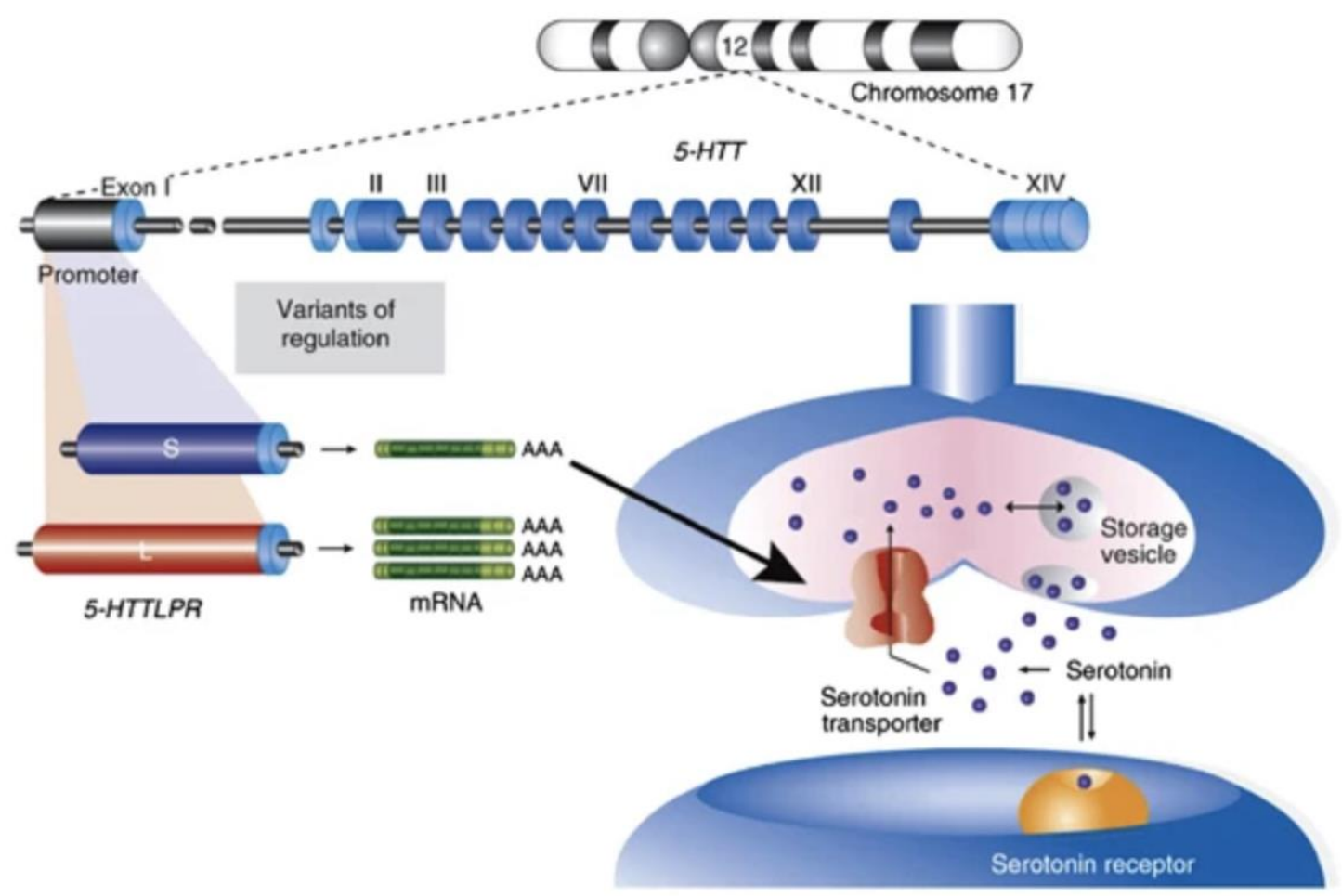

Figure 1.4: Presentation of the two human variants of the serotonin transporter and their role in serotonin signalling. Polymorphisms in the promoter region of the serotonin transporter on Chromosome 17 produce different rates of transcription, leading to altered expression of the serotonin transporter. Figure sourced from De Neve, 2011 (Reference 144), with permission to reprint from Springer Nature (license 5146800010192). 


\subsection{The RAC1/KAL7/DISC1 Signalosome}

\subsubsection{RAC1 Activity}

RAC1 is a small monomeric $\mathrm{G}$ protein, one of the most well characterised members of the Ras superfamily of GTPases [149]. These proteins are key regulators in signal transduction pathways throughout the cell, acting as 'molecular switches' (Fig 5). GTPases are bound to a guanine diphosphate (GDP) molecule while inactive and undergo a confirmational change and become activated when a Guanine Exchange Factor (GEF) facilitates the replacement of GDP with guanine triphosphate (GTP). GTPases remain in an active state until their intrinsic GTPase activity converts the bound GTP molecule to GDP; this is often expediated by the association of a GTPase activating protein (GAP). This cyclical process allows not only tight regulation of cellular signalling, but a reversible and plastic system that can respond relatively rapidly to a multitude of upstream regulatory signals [149]. RAC1 activity specifically is associated with regulation of the actin cytoskeleton. In the brain, this includes axon growth and guidance as well as dendrite morphology and synaptic strength [150].

\section{Figure 1.5: Cyclical Activation of GTPases}

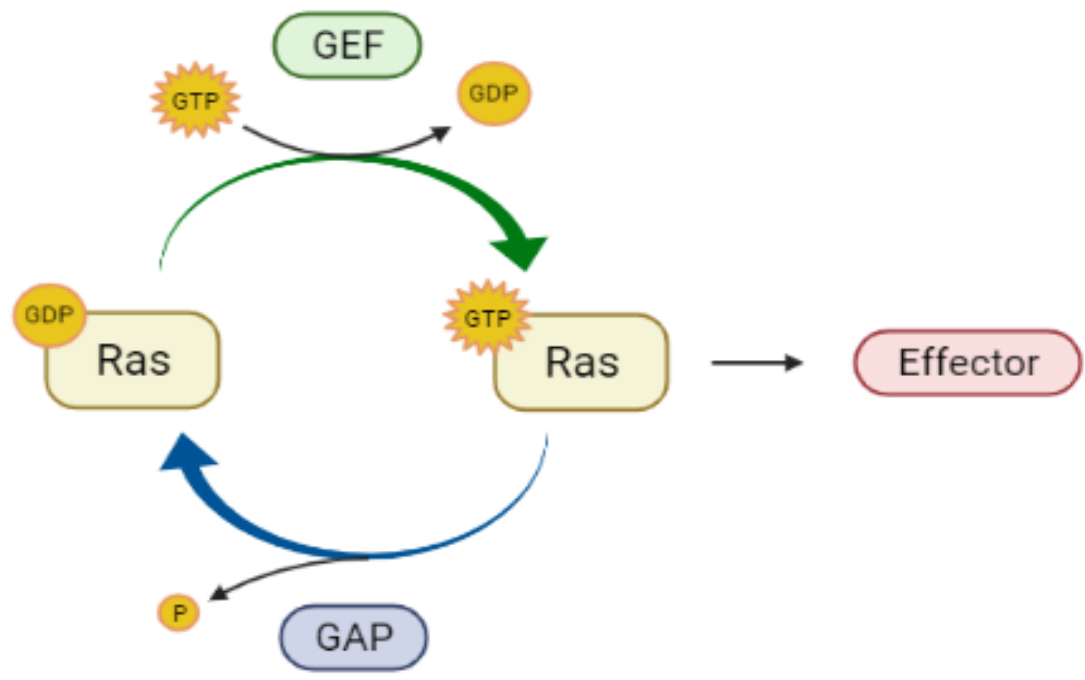

Figure 1.5: A simplified presentation of Ras GTPase activity within the cell. Binding of GTP on association with a Guanine Exchange Factor (GEF) causes GTPase activation, allowing downstream signalling to occur. GTPase Activating Proteins (GAP) facilitate GTPase activity, resulting in conversion of GTP to GDP and subsequent inactivation. This prevents signalling through this pathway until re-association with a GEF. Image created on BioRender.com, based on Figure 1 from Hall et al. 2010 (Reference 151). 
During neurogenesis, RAC1 has been associated with axon growth and guidance [151]. Neuronal growth cones extend and migrate through the formation of filipodia and lamellipodia structures, reliant on restructuring of the actin cytoskeleton. Typically, RAC1 localises to the growth cone of immature neurons and mediates actin polymerisation, resulting in the production of protruding filipodia structures [152]. This activity works I conjunction with other GTPases CDC42 and RHOA, which contribute to the production of lamellipodia and depolymerisation of actin polymers respectively [153]. Interestingly, both constitutively activated and knockout models of Rac1 in chick embryonic neurons show aberrant growth cone and neurite morphology; this suggests not only Rac1 activation, but cyclical Rac1 activity, is required for normal neuronal growth [154]. Rac1 deletion in mouse forebrain results in similarly altered neuron growth, as well as common cell death and misrouting of neurons into abnormal brain regions [155]. Abnormal directional growth and morphology of neurons directly results in altered connectivity between brain regions, making RAC1 an important factor of interest in NDD theories of connectivity.

RAC1 is also highly implicated in synaptic stability and strengthening. During periods of low signalling, inactive RAC1 is localised to cytosolic pools within the cell. Activation by NMDA receptors has been shown to promote transport of RAC1 to the plasma membrane, where it can subsequently be activated in the correct sub-cellular compartment for downstream processes such as long term potentiation (LTP) [156]. LTP results in the enlargement of the post-synaptic density, along with an increased expression of AMPA receptors at the membrane to increase synapse sensitivity during high levels of excitatory signalling. Extended activation of AMPA and NMDA receptors triggers the beginning the LTP process, initiation of which has been shown to require RAC1. Active RAC1 subsequently modulates the cytoskeleton through downstream interaction with cofilin, an actin depolymerising protein. RAC1 signalling through secondary factors such as PAK1 primarily inhibits cofilin and prevents actin depolymerisation, promoting growth. Overactivation of this pathway has been demonstrated to cause abnormal dendritic morphology in a model of the common ASD comorbidity Fragile X Syndrome [157]. RAC1 has also been implicated in long term depression (LTD) of hippocampal synapses, with inhibition of Rac1 in animal studies resulting in LTD deficits [158]. Altered synaptic plasticity is also a key feature of NDD pathology, suggesting disrupted RAC1 activity may play a large role in NDD development. 


\subsubsection{Regulation by KAL7 and DISC1}

The RAC1/KAL7/DISC1 signalosome is a well-reported mechanism of RAC1 regulation within the synapse (Figure 1.6). Kalirin-7 (KAL7) is a GEF protein that facilitates activation of RAC1, encoded in humans by the KALRN gene [159]. Evidence suggests KAL7 is a main regulator of RAC1 in the brain: Overexpression of Kal7 in cultured mouse neurons has been shown to increase dendritic spine formation, while Kal7 knockout mice show reduced spine and synaptic density is observed [160, 161]. LTP can still occur without KAL7 expression however to a lesser extent, suggesting other RAC1 activators such as KAL7 paralog TRIO may be able to continue LTP processes [161-163]. Alterations in KAL7 alone are rarely directly associated with ASD and SCZ; one study reports reduced expression in the prefrontal cortex of SCZ individuals under the KAL7 alias DUO [164]. Mutations in the KALRN gene have recently been associated with both ASD and SCZ [159]. These alterations tend to effect function of splice variants such as KAL9, the primary form of $K A L R N$ expressed during neurodevelopment [165].

Disrupted in Schizophrenia-1 (DISC1) acts to prevent KAL7-facilitated activation of RAC1 by anchoring KAL7 to scaffolding protein PSD95, sterically inhibiting access to the active site of KAL7 (Figure 1.6). In an extensive study by Hayashi-Takagi et al, DISC1 was demonstrated to not only reduce RAC1 activation and increase co-localisation of KAL7 and PSD95, but that this localisation was reduced upon NMDA receptor activation [166]. Other studies of this pathway have suggested this may additionally be influenced by other NDD related proteins such as neurexin/neuroligin complexes [167]. DISC1 has a long history of implication in SCZ [168]. An early Scottish pedigree study indicated mutations in DISC1 and DISC2 with increased incidence of SCZ and MDD [169]. DISC2 encodes a long non-coding RNA antisense to DISC1, hypothesised to regulate DISC1 expression [170]. DISC1 has also been directly associated with ASD in a Finnish cohort [171]. In MIA studies, DISC1 mutations have been associated with NDD-like behavioural changes and increased susceptibility to MIA characterised by increased IL-6 [172]. 


\section{Figure 1.6: Simplified View of the RAC1/KAL7/DISC1 Signalosome}
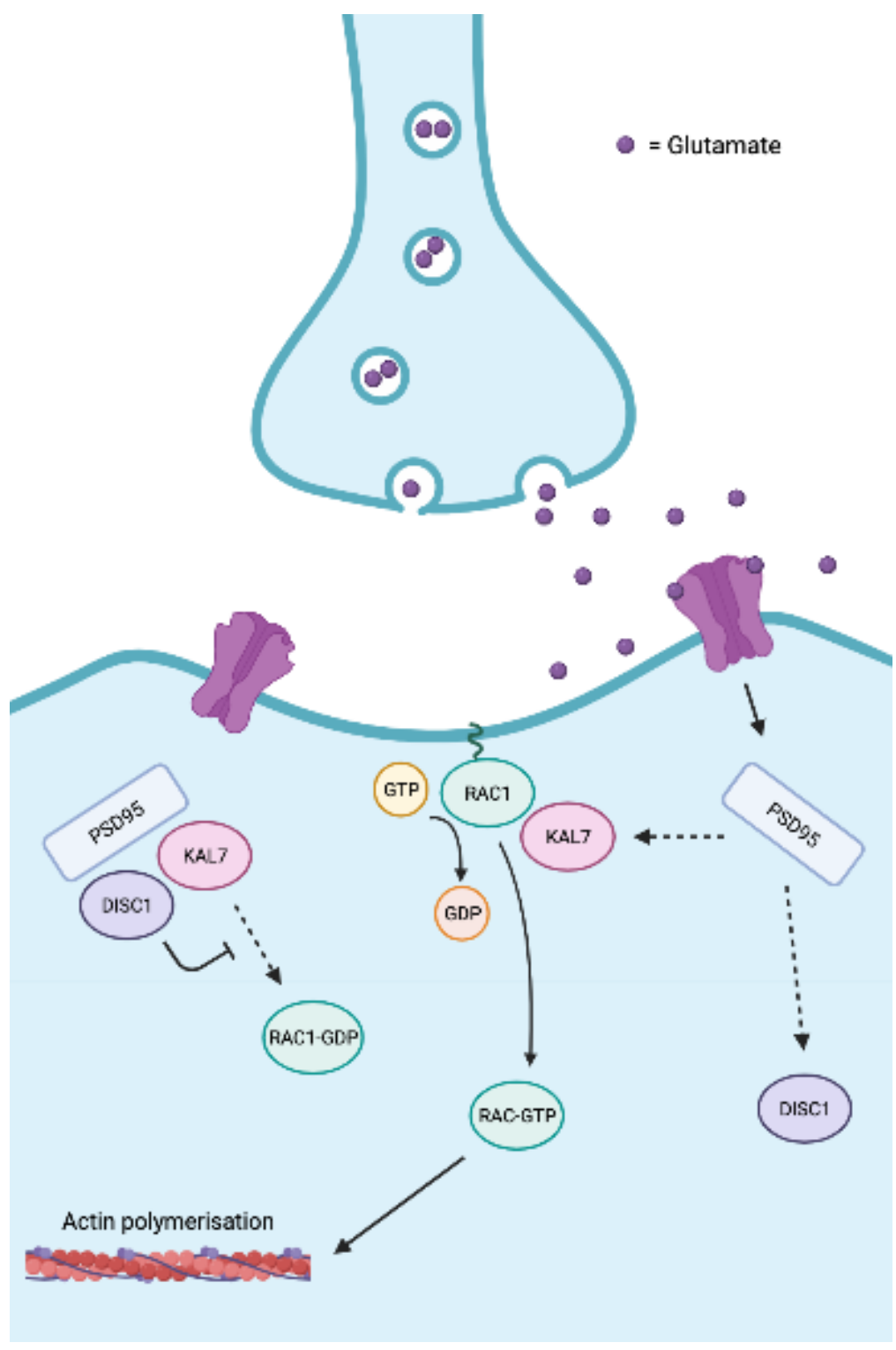

Figure 1.6: Activation of NMDA receptors by glutamate signalling induces RAC1 associated actin remodelling. NMDA receptor activation results in dissociation of the PSD95/DISC1/KAL7 complex and localisation of RAC1-GDP to the membrane, allowing KAL7 to associate with Rac1. GTP is subsequently bound to RAC1 and activates downstream polymerisation of actin monomers through pathways such as PAK1 (not shown). Image created on BioRender.com.

\subsection{Aims of this Research}

The overall focus of this project is to investigate the effect of normal or reduced SERT expression in the maternal immune activation model of neurodevelopmental disorders 
(Figure 1.7). A model of viral mimic-induced maternal immune activation will be used via administration of Poly I:C on GD12.5, targeting the experimental window of both SCZ and ASD research (Figure 1.3). This will be applied to a Wistar SERT knockout rat model, where pups will either be wildtype (WT) or heterozygous (HET) for the serotonin transporter to mimic expression variation in humans. This combination of genetic and environmental risk factors has received minimal research; while effects of poly I:C on SERT expression have been reported [173], to the best of our knowledge no published research has administered poly I:C to a SERT knockout model. Based on the known effects of MIA and SERT function independently in NDDs, it is hypothesised that reduced SERT expression in HET pups will increase susceptibility to MIA-induced changes in offspring. Both male and female pups will be assessed and compared in this model, as sex-specific differences are well reported in human and animal NDD research and should be explored further [174]. This gives a total of 8 groups of pups to be compared, considering each treatment/sex/genotype group as a unique cluster to compare across experiments.

To investigate the effects of this model, two avenues will be explored; ultrasonic vocalisations will be measured and characterised to investigate behavioural phenotype, while expression of the Rac1/Kal7/DISC1 signalosome in the hippocampus will be measured to assess changes in synaptic plasticity. Behavioural tests will be carried out at postnatal day (PND) 7, when brain development and communication most closely resemble the developmental stage of humans in early childhood. Molecular analysis will be carried out at PND21, a developmental stage equivalent to human adolescence/young adulthood. Timing was decided to encompass developmental stages critical to ASD and SCZ. Maternal immune activation alone is reported to affect both of these areas, and it is expected that changes will be seen in both regardless of genotype effect. Furthermore, SERT HET offspring exposed to poly I:C are hypothesised to show the strongest divergence from controls across one or both avenues. 


\section{Figure 1.7: Graphic Representation of Overall Experimental Design}

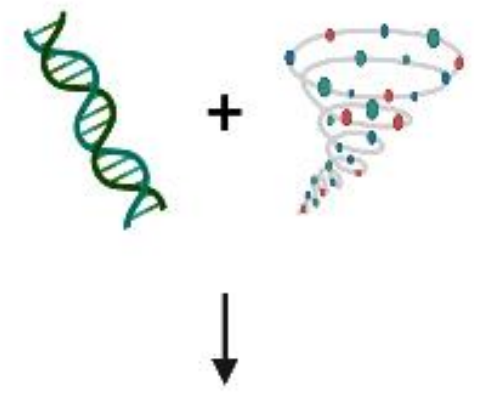

Model Development:

Genetic alteration and maternal immune activation
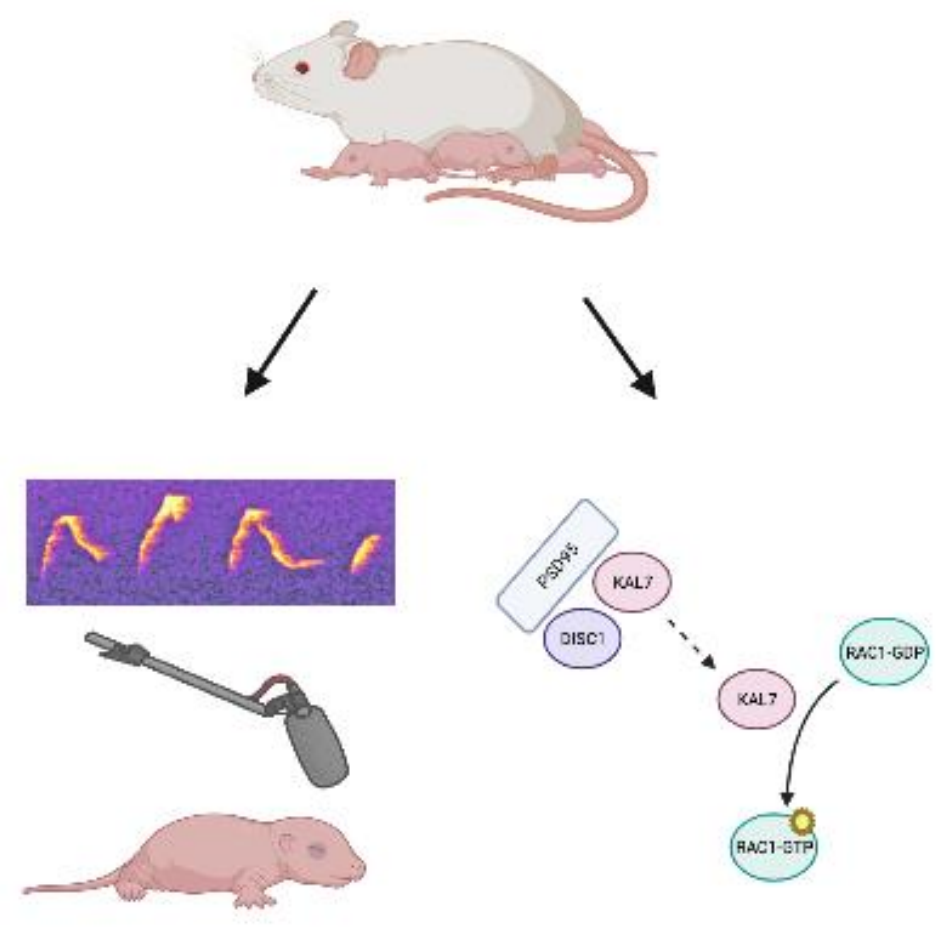

Model Validation: maternal tests and offspring growth, maturation until PND21

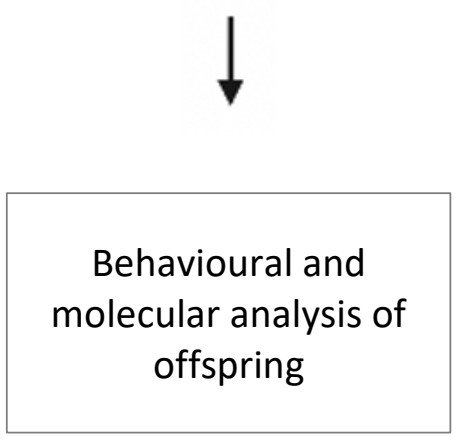

Figure 1.7: An overview of the study carried out in this thesis. Genetically reduced expression of the serotonin transporter and maternal immune activation are used in conjunction to affect rat pup foetal development. Before pup analysis, the model will be validated to ensure maternal immune activation was successful. Offspring changes induced by this model will be assessed behaviourally through recording of ultrasonic vocalisation (left) at PND7 and expression of signalling pathway components linked to neurodevelopmental disorders at PND21 (right). Image created on BioRender.com. 


\section{Chapter 2. Methods}

\subsection{Animals}

All rats used in this study are either Wistar or Wistar-bred serotonin transporter (SERT) knockout animals. The SERT knockout allele in this model contains a $\mathrm{C}$ to $\mathrm{A}$ transversion mutation at position 3924 in the SERT gene, resulting in a premature stop codon [175]. This allele is subsequently transcribed but not translated, with interrupted transcripts being degraded by nonsense-mediated decay. This was verified in the initial study characterising the SERT knockout model [175]. Our lab has also recently verified this through High Resolution Melt Analysis (HRMA); DNA samples from SERT +/- animals have both wildtype and knockout alleles present, however reverse transcribed cDNA samples only present wild type transcript and at an abundance less than $50 \%$ of WT animals (unpublished results).

In total, 18 breeding animals and 70 pups were involved in this study (Table 2.1). All mating was carried out using SERT HET females bred on a Wistar background, and Wistar stock males (the equivalent of SERT WT). SERT WT males were originally planned for the breeding pairs in this study, however were substituted with readily available Wistar males due to time constraints related to the Covid-19 pandemic. All animals were housed in standard polycarbonate cages in an individually ventilated caging (IVC) system under a reverse-light cycle (dark between 7am-7pm). Additionally, all housing rooms were temperature and humidity-controlled $\left(21^{\circ} \mathrm{C} \pm 2^{\circ} \mathrm{C}\right.$ and $55-60 \%$ respectively). All animals in this study had access to food and water ad libitum for the entire experimental period.

Dams ( $n=13)$ were semi-randomly assigned to a treatment group; littermates were separated across treatment groups when used, and dams in final breeding rounds had predetermined treatment group to replace mothers/litters previously excluded from analysis (resulting in $\mathrm{n}=3$ litters per treatment). Pairing was semi-random also, only controlled for by limiting pairing of fathers $(n=5)$ to a maximum of one dam per treatment group to prevent closely related litters confounding results. As the study progressed and litters were excluded 
(see Chapter 3), previously paired fathers were re-paired with new, untreated females to minimise the number of breeding males associated with the study.

Of the 13 breeding pairs established, 10 produced litters available for study. Pups were housed with the mother following birth until either weaning (for the PND60 cohort) or sacrifice on PND21. Pups were handled minimally, however between PND7-21 regular handling was required to re-apply identification markings (see section 2.3). Pups assigned to the PND60 cohort were given ear tags for identification on PND21 before rehousing with 2-3 same-sex littermates, and not handled for experimental purposes again until sacrifice.

Pup exclusions were made for dam, litter, and genotype related reasoning; these will be elaborated on in further discussions. In total, 71 pups of known genotypes from the 6 approved litters were included in this study. All 70 were used in USV analysis, however only a subset were used in RT-qPCR analysis (Table 2.2). Pups were predominantly sacrificed at PND21 ( $n=49)$, with a small group sacrificed at PND60 for further exploratory study $(n=22)$. Stratified sampling was used to select a minimum of two pups from each treatment/sex/genotype group for RT-qPCR analysis $(n=22)$, with pups within a group selected from alternating litters ( $n=3$ per treatment) to minimise the chance of litter-based variation producing misleading results.

With the exception of standard housing changes and upkeep, all animal handling and experimental procedures were carried out by myself or in concert with another lab member following thorough training approved by animal facility staff. This consistency was aimed to reduce any effects in behaviour or development attributable to varied or overly stressful handling experiences [176]. All animal work for this thesis was carried out following ethics approval from the Victoria University of Wellington Animal Ethics Committee (Ethics Number: 25848). 
Table 2.1: Total Breeding Animals and Pups Associated with this Thesis

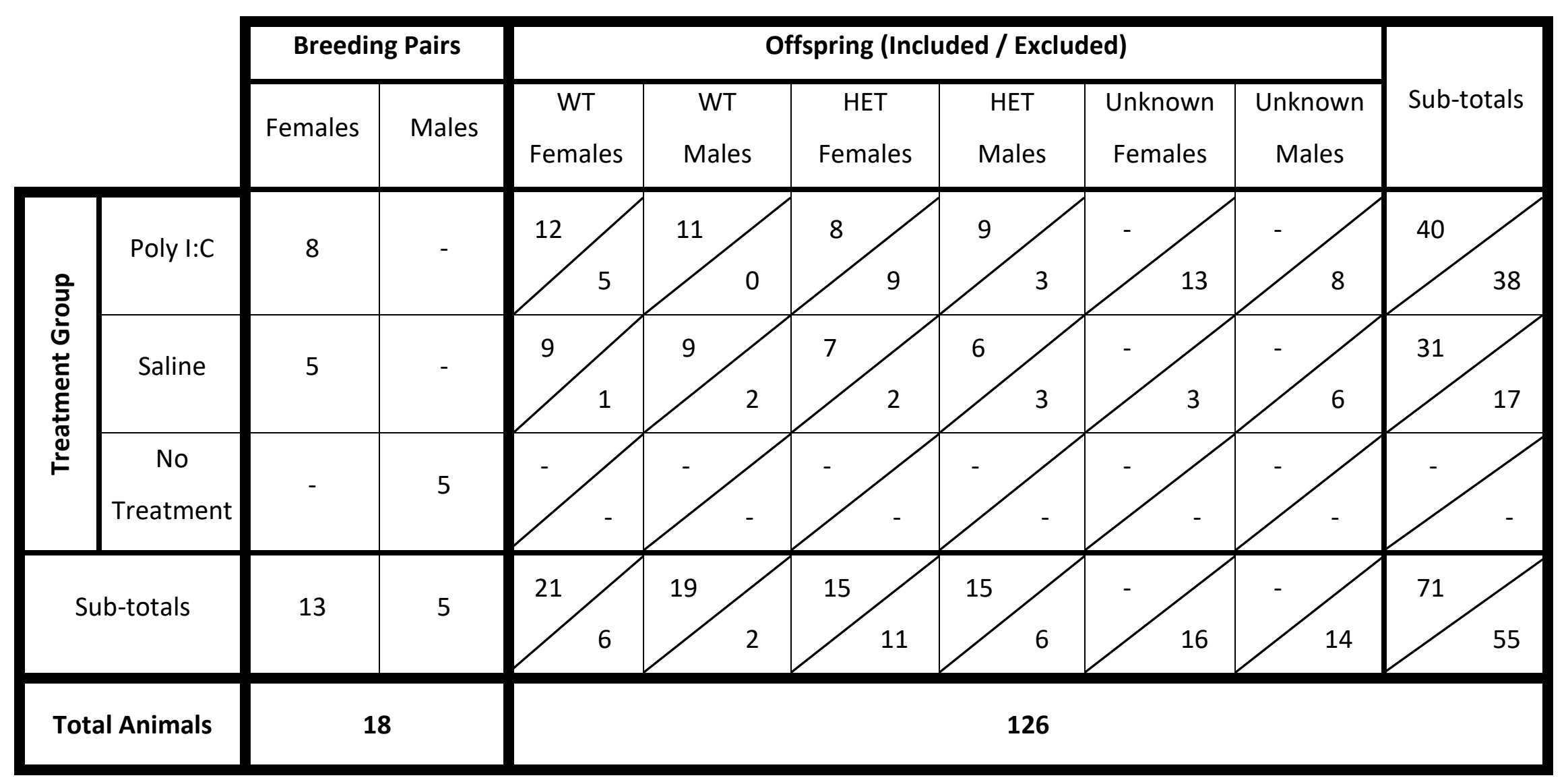

Table 2.1: The number of animals associated with each treatment group is presented by row, and differentiated by sex and genotype groupings by column where relevant. Horizontal dashes indicate a category is not applicable. A total of 18 breeding animals and 126 pups were associated with this project. Only 71 available pups were used in downstream experiments, with a total of 55 pups excluded from analysis. Exclusions were made based on the results of validation tests (see Chapter 3) or inconclusive genotyping results (see section 2.6). 


\section{Table 2.2: Eligible Pups Used or Awaiting Use in RT-qPCR Experiment}

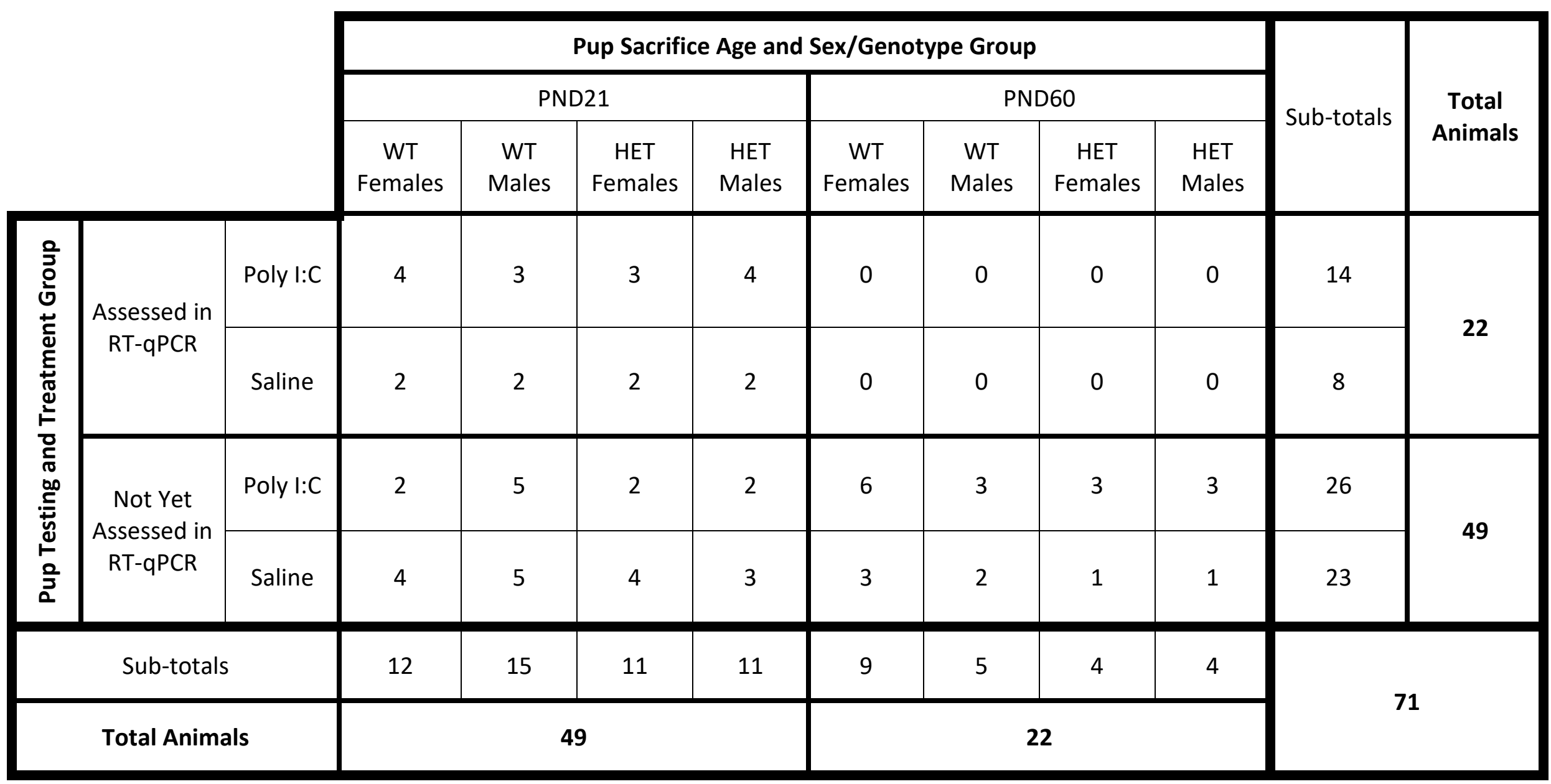

Table 2.2: Pups accepted for use in this study are presented based on sacrifice age and selection for RT-qPCR analysis, further separated by treatment and sex/genotype groups. Pups were sacrificed at either PND21 or PND60. No PND60 pups have been analysed in the experiments in this thesis, while 22/49 of the PND21 cohort were used. 


\subsection{Breeding Program}

Wistar SERT HET females that had not previously been mated were paired with stock Wistar males in a standard timed breeding program. Home cages were equipped with metal grid floors and checked every $24 \mathrm{~h}$, for a maximum of 5 days consecutively, for vaginal plugs. Discovery of at least one plug was considered evidence of successful mating and designated GD0.5. Pairs were separated at this time to prevent any late pregnancies and therefore inaccurate treatment timing. Males were re-housed with others where appropriate, while pregnant females were singly housed until birth. Unsuccessful pairings were separated after 5 days and repaired for another attempt following a mandatory separation period of 7 days. Females which received an injection but did not produce a litter were not used again $(n=3)$, in case of behavioural change to injection or altered immune response to a second challenge in the case of poly I:C exposure $(n=2)$. These animals were allocated to other projects where possible to reduce extra animal use as according to ethics guidelines.

\subsection{Maternal Treatments}

Pregnant dams were treated on GD12.5 via subcutaneous injection with 27-gauge needle behind the shoulders. Dams were restrained in the hand or with the use of a towel as per standard operating procedure guidelines. Experimental group dams were injected with a $5 \mathrm{mg} / \mathrm{kg}$ dosage of Poly I:C solution, at a volume of $1 \mathrm{ml} / \mathrm{kg}$. Poly I:C solution was made using Poly I:C powder (P1530, Lot \#116M4039V, Sigma-Aldrich) fully dissolved in $0.9 \%$ saline vehicle, at a concentration of $5 \mathrm{mg} / \mathrm{ml}$. Control group dams were given $1 \mathrm{ml} / \mathrm{kg}$ volume injections of $0.9 \%$ saline vehicle from the same stock used to produce Poly I:C solution.

Dam temperature and weight measurements were made immediately prior to injection. Measurements were then repeated at 3, 6, and 24 hours post injection. Temperature measurements were always taken by the same handler, and immediately upon handling to reduce temperature increase variation due to increased stress. These were measured using a handheld infrared thermometer. Weight measurements were taken directly post-temperature measurement before return to home cage. 


\subsection{Maternal Separation Test}

The home cage was transferred to a soundproof room for audio recording, under reverse-light cycle to match home environment. PND7 pups were removed singly from the home cage and placed within a custom-built polystyrene chamber with a microphone (UltraMic 250K, 16 bit) suspended above through a hole in the chamber lid (Figure 2.1). 5minute recordings of each pup on Audacity software (Audacity Team, version 2.4.2) were recorded immediately after separation. Pups were then weighed on a pre-warmed scale for weight change analysis. Each pup was subsequently assigned a number, written on the back with a non-toxic permanent marker before return to the home cage. Numbers were reapplied as needed every 1-3 days, transferring location from back to tail at approximately PND13 when tails become rougher and hold ink. This continued until weaning at PND21, to allow tracking of each pup until tissue collection. Each pup was tested sequentially, before return of the home cage to previous location. 


\section{Figure 2.1: Maternal Separation Test Equipment}

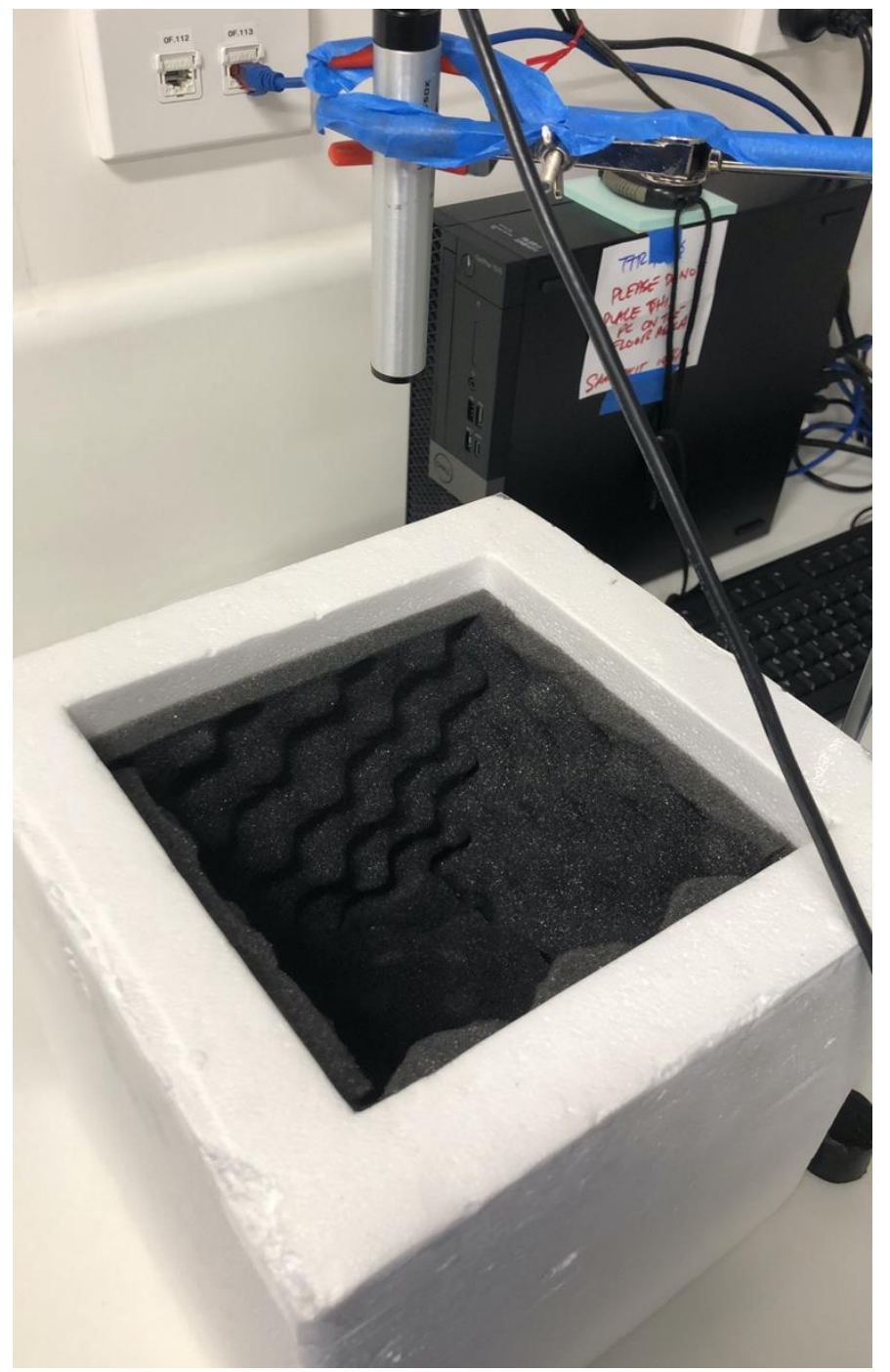

Figure 2.1: The base of the polystyrene chamber and the microphone used in maternal separation tests. The inside of the chamber is padded to prevent sound entering and leaving the chamber other than upwards toward the microphone. Lid of the chamber is not pictured. 


\subsection{Analysis of Ultrasonic Vocalisations}

\subsubsection{Sound Editing}

Due to self-noise from some microphones used, selective editing of Litter 5 audio files was used to remove background interference before call analysis. Audacity (Audacity Team, version 2.4.2) was used to view spectrogram files, where selection and removal of constant background frequencies was achieved using the spectral delete tool (Figure 2.2) This removed constant background noise interfering with call detection, however may result in a slight loss of call contour where crossing the contaminated frequency band.

\section{Figure 2.2: Sound Editing in Audacity}

A

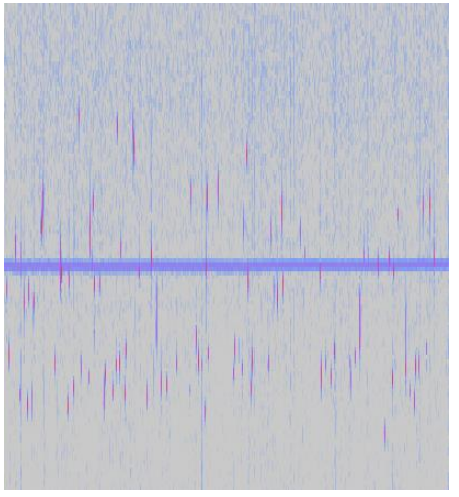

B

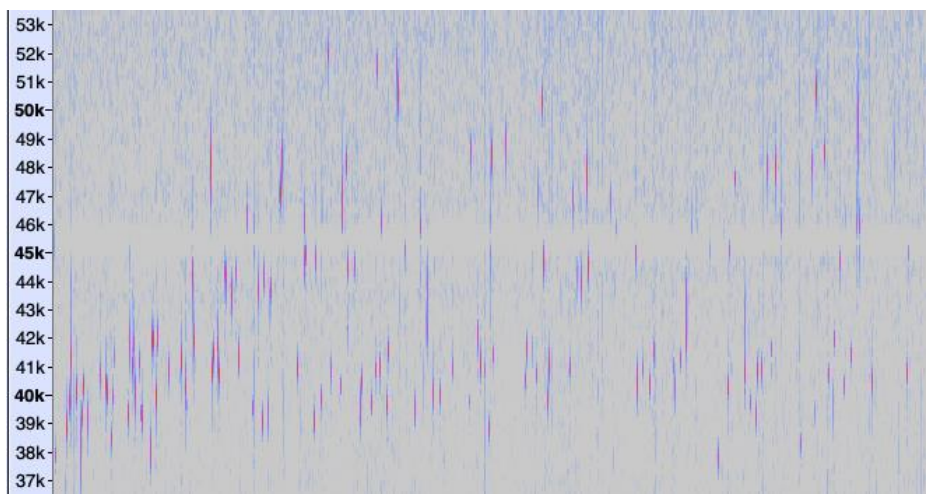

Figure 2.2: Audio files from Litter 5 before (A) and after (B) sound editing. A) An audio file with a band of constant sound contamination at $45.5 \mathrm{kHz}$, within the range of pup calling during maternal separations (represented in pink). B) the same audio file with sound contamination removed. Almost all background audio is removed from the targeted band, however some call contours remain. 


\subsubsection{Call Identification}

Audio files were input into DeepSqueak (Coffey and Marx, version 2.6.1) run through MATLAB (MATLAB, version 2018a). Detection files were then generated using a neural network designed for pup ultrasonic vocalisation identification, developed in Dr Ellenbroek's group. Detections were manually assessed using the extended program fork DeepSqueak Screener (UEFepilepsyAIVI, version 0.9) in MATLAB (MATLAB, version 2019b). Manual correction of false positive, false negative, and inaccurate call contours was carried out on every file (Figure 2.3), using the following guide parameters:

a) Calls must be longer than 10 milliseconds (ms), to exclude 'clicks' and blips with no identifiable shape.

b) Calls must be more than $25 \mathrm{~ms}$ apart, to prevent small gaps from sound editing or animal movement/volume loss creating two calls where there should be one.

c) Calls must reach a minimum amplitude of 0.15 decibels $(\mathrm{dB})$ to ensure clear call shape is identifiable (represented by a blue shade on 'cubehelix' colour view setting, which provides the most visual clarity). This rule was reduced to $0.1 \mathrm{~dB}$ for Litter 1 files as a necessary microphone change reduced amplitude and volume of all noise recorded (see Chapter 4.3 for discussion).

d) Call contour must represent the visual shape of a call and only the call, excluding any contaminating background noise. 


\section{Figure 2.3: DeepSqueak Call Detections Viewed in DeepSqueak Screener}

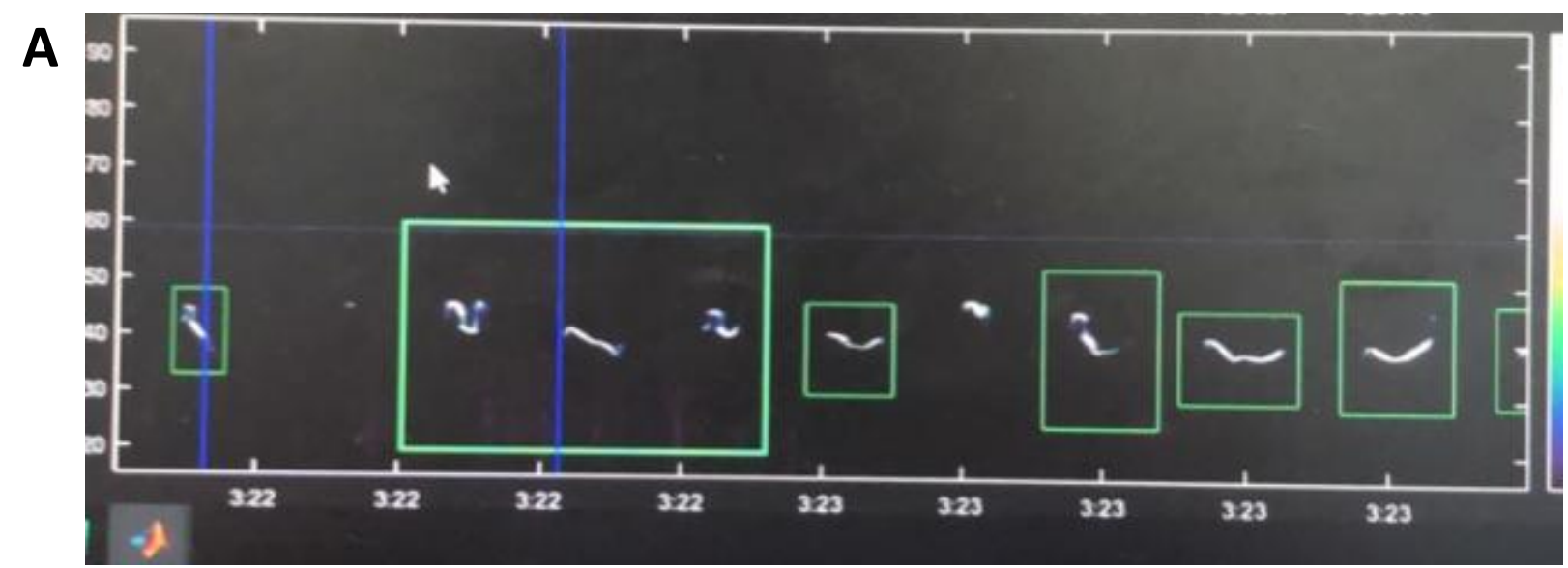

B

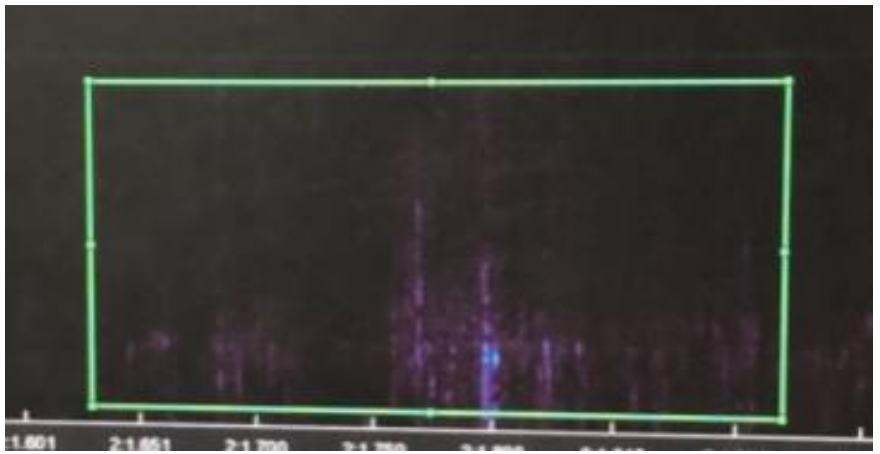

C

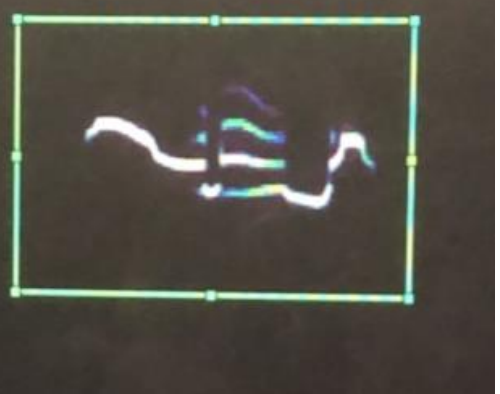

Figure 2.3: Example of DeepSqueak call detection errors and the range of calls present. A) One second segment of a 5-minute pup recording analysed by DeepSqueak, viewed in DeepSqueak Screener showing common detection errors. One call was not identified by the detection network, and three calls were misidentified as one. B) An example of background noise in isolation without a call, misidentified as a call by the detection network. C) A correctly identified call with a highly complex shape compared to common calls (A). 


\subsection{Tissue Collection}

\subsubsection{Tail Tips}

PND11/12 pups were held in the palm of the hand, with tails held in ice cold $70 \%$ ethanol for approximately 20 s to numb area. Approximate $1 \mathrm{~mm}$ section at tip of tail were removed via scalpel blade and immediately placed in microcentrifuge tube for genotyping. Pups were then placed in a temporary cage while the litter sampling was completed, to reduce maternal stress due to any blood on returned pups and continued removal of pups while sampling continued. Mother was kept with the bulk of pups at all times, being moved to the temporary cage after over half the litter was sampled.

\subsubsection{Brain Dissections}

The majority of dissections were performed at weaning on PND21, with a small subset left for PND60 for future studies (Table 2.2). All pups were weighed on PND21 prior to dissection or re-housing for weight change analysis. Animals were euthanised with carbon dioxide gas prior to decapitation and brain extraction. Extracted brains were placed in appropriately sized 3D printed brain blocks and washed in PBS (Figure 2.4A). Razor blades were placed $1 \mathrm{~mm}$ into the prefrontal cortex and at the beginning of the cerebellum first to secure section. Day 21 brains had further blade placements at approximately $2 \mathrm{~mm}$ and $4 \mathrm{~mm}$ back from front blade - this varied slightly depending on brain size. Sections from these were used to dissect the frontal cortex (Figure 2.4A-C), hippocampus (Figure 2.4A, B, D), and cerebellum (Figure 2.4A). Incorrect blade placement resulting in hippocampal tissue split between two sections was recovered from both where confidently identified (Figure 2.4A) to maximise tissue recovered. Day 60 were dissected in the same method, with blade placement shifted approximately 1-2mm depending on brain size. Only hippocampus samples were used in this study, with frontal cortex and cerebellum sampled for related projects. Dissected samples were flash-frozen on dry ice and subsequently stored at $-80^{\circ} \mathrm{C}$ until use. 


\section{Figure 2.4: Dissection of Brain Tissue}
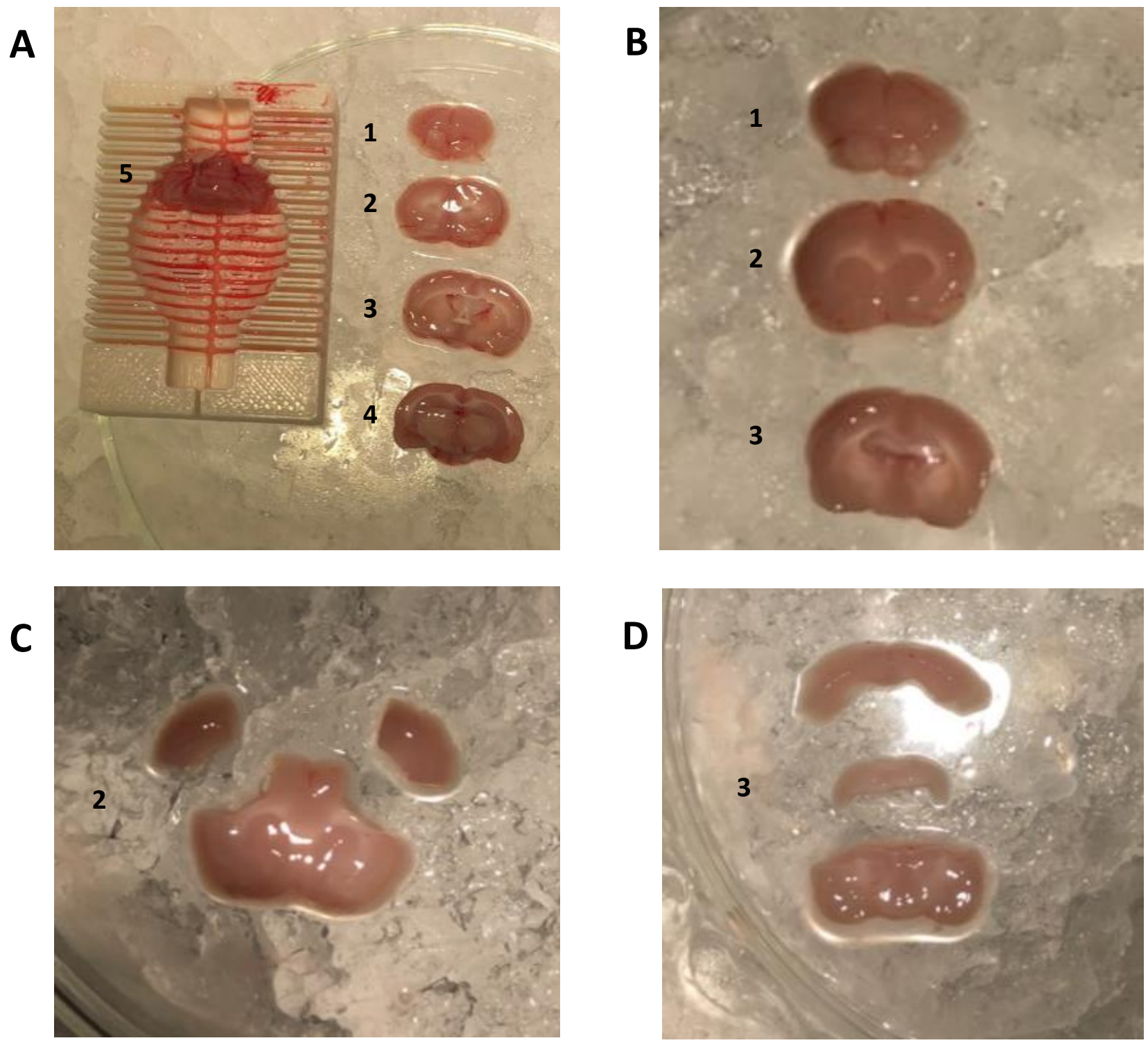

Figure 2.4: Examples of dissected brain regions used. A) A whole PND60 brain was sectioned into 5 regions; $1=$ olfactory bulb and prefrontal cortex, 2=frontal cortex section, 3= main hippocampal section, 4= remaining brain tissue, $5=$ cerebellum section. Note section 4 in this example contains posterior hippocampal tissue, present in two grey segments beneath the corpus callosum (white), which was also collected for analysis. B) Corresponding segments were taken from a PND21 brain, with sections 4 and 5 not pictured. Sections 2 and 3 are micro-dissected in the following images (C-D). C) Section 2 is pictured with extracted frontal cortex segments used for analysis. Scalpel blade was used to cut above the corpus callosum to dissect left and right frontal cortex regions, which were stored in the same microcentrifuge tube to jointly comprise frontal cortex samples. D) Section 3 is pictured with hippocampus region (middle tissue segment) isolated. Whole hippocampus samples were micro-dissected by scalpel and stored in one microcentrifuge tube. 


\subsection{Genotyping}

\subsubsection{Primer Design}

Primers for genotyping by High Resolution Melt Analysis (HRMA) were designed previously in this laboratory to target the single nucleotide polymorphism on the knockout allele of SIc6a4 (Table 2.3). SERT1 is the primary primer set used for all genotyping, with SERT3 as a secondary set used to confirm results when required.

Table 2.3 Genotyping Primer Sets

\begin{tabular}{|c|l|c|c|}
\hline \multirow{2}{*}{ Target } & \multicolumn{1}{|c|}{ Primer Sequence } & $\begin{array}{c}\text { Melt Temp } \\
\left({ }^{\circ} \mathbf{C}\right)\end{array}$ & $\begin{array}{c}\text { Amplicon Size } \\
\text { (base pairs) }\end{array}$ \\
\hline \multirow{2}{*}{ SERT1 } & FW: TAC CTC ATC TCC TCC CTC AC & 55.0 & \multirow{2}{*}{96} \\
\cline { 2 - 3 } & REV: GTT GTC CTG GGC GAA GTA G & 55.5 & \\
\hline \multirow{2}{*}{ SERT3 } & FW: GCA CGA ACT CCT GGA ACA & 55.1 & \multirow{2}{*}{75} \\
\cline { 2 - 3 } & REV: TGG AAT GCA GCG TCC AG & 55.5 & \\
\hline
\end{tabular}

Table 2.3: Primer sequences used for genotyping in this study. Both primer sets were designed and validated by Bryony Thorne for use across research projects using SERT knockout animals.

\subsubsection{Polymerase Chain Reaction}

To prepare samples for polymerase chain reaction (PCR) amplification, tail tips taken at PND11-12 were fully submerged in 20 $\mu$ I DNA Extraction Reagent (Quantabio, Extracta DNA Prep Kit \#95091-025). In cases of parental genotyping, ear punch samples were taken following euthanasia and used instead. Submerged samples were then incubated at $95^{\circ} \mathrm{C}$ for 30 minutes, vortexed, and placed on ice for subsequent addition to PCR reaction.

PCR reactions were constituted in $10 \mu$ l volumes. Each reaction contained $1 \mu$ l of extracted DNA, $5 \mu$ I Accumelt HRM SuperMix (Quantabio, \#95103-250), 0.2 $\mu$ M concentration of forward and reverse primers, and remaining volume in nuclease-free water. All genotyping 
PCRs also contained a negative no template control with no DNA added, to ensure contaminating DNA was not confounding results. Reactions were then placed in a Bio-Rad CFX96TM Real-Time PCR Detection System thermocycler (Bio-Rad, \#185-5096) for amplification. PCR conditions were as follows: initialisation at $95^{\circ} \mathrm{C}$ for $2 \mathrm{~min}$, preceding 35 cycles of $15 \mathrm{~s}$ at $95^{\circ} \mathrm{C}$ (denaturation), $15 \mathrm{~s}$ at $52^{\circ} \mathrm{C}$ (annealing), and $15 \mathrm{~s}$ at $72^{\circ} \mathrm{C}$ (elongation). Melt curves were generated after amplification, by increasing temperature across a $70^{\circ} \mathrm{C}$ to $90^{\circ} \mathrm{C}$ range at $5 \mathrm{~s}$ for each $0.2^{\circ} \mathrm{C}$.

\subsubsection{High Resolution Melt Analysis}

Using the melt curves produced following amplification, subtle differences can be detected in PCR products between reactions by shifts in melt temperature. Melt temperature is determined over a range of temperatures by the peak in fluorescence resulting from denaturation of PCR product strands. The $\mathrm{C}$ to $\mathrm{A}$ transversion present in the knockout allele of the SERT gene in this model results in the replacement of a C-G bond with an A-T bond. The latter requires less energy to denature, resulting in a product with a slightly lower melt temperature. By using a high-resolution melt temperature range with minimal increments $\left(0.2^{\circ} \mathrm{C}\right)$ and a short PCR product $(<100 \mathrm{bp})$, this subtle difference in alleles is detectable by melt analysis. WT pups appear as the sharp peak with the highest temperature, HOMs as a sharp peak with the lowest temperature, and HETS with a broad peak at a middle temperature (caused by the reaction containing both alleles as double-stranded products and mismatched allele strands annealing, resulting in a wide range of product melt temperatures).

Analysis on Bio-Rad Precision Melt Analysis Software (Bio-Rad Laboratories, version 4.0.52.0602) was carried out to clarify the differences between genotypes for pup identification. Cluster analysis of normalised melt curves identify almost all pups in this study as WT or HETs, with a small subset of 17 tested pups across treatment groups and litters found inconclusive (Figure 2.5). Repeated PCR analyses, re-sampling from dissected brain tissue, and change of primer set all fail to identify this cluster as either WT or HET (data not shown). Additionally, parental genotyping confirmed all mothers as SERT HETs and fathers as WT (Appendix 1). Due to time constraints the genotype of the unknown cluster of pups has not 
yet been identified, however this process is ongoing. As these pups are currently of unknown genotype, they have been excluded from the study (Table 2.1). 


\section{Figure 2.5: Genotyping of Pups Using High Resolution Melt Analysis}
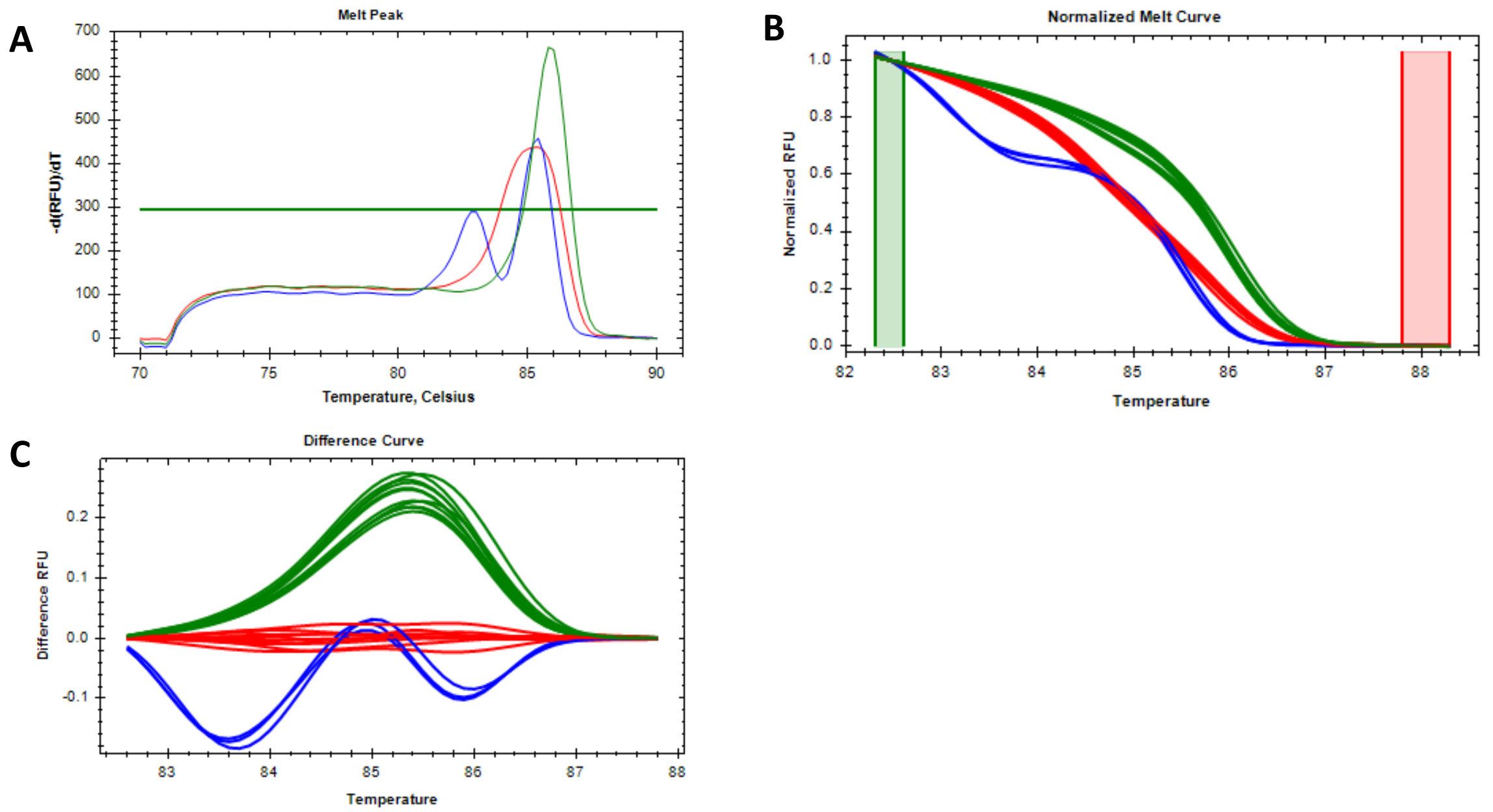

Figure 2.5: Genotyping by High Resolution Melt Analysis reveals three discrete groups of pups. All melt curves shown result from pups in litters 2 and 7, with results representative of all pups in this study. Colouring is consistent across graphs; green as WT, red as HET, and blue is unknown. A) Example melt curves generated by qPCR show a tall sharp WT peak at approximately $86^{\circ} \mathrm{C}$, and a wider HET peak slightly lower at approximately $85.5^{\circ} \mathrm{C}$. A third group consists of two peaks, with the second peak sharp and at the lower temperature of $85.5^{\circ} \mathrm{C}$. B) Normalised melt curves show 3 consistent clusters across the litter. C) Difference curves derived from normalised melt curves (B) show separated clustering of all pups in both tested litters, normalised with the red HET cluster at 0. 


\subsection{Quantitative Reverse Transcription Polymerase Chain Reaction (RT-qPCR)}

\subsubsection{Primer Design}

Primer sets designed and used in this study are presented below (Table 1). All primer sets were assessed for self-complementarity and specificity through Primer-BLAST. Primers were either sourced from relevant literature or designed specifically for this project using the PrimerQuest tool (Integrated DNA Technologies, 2012).

$\underline{\text { Table 2.4 RT-qPCR Primer Sets }}$

\begin{tabular}{|c|c|c|c|c|}
\hline Target & Primer Sequence & $\begin{array}{l}\text { Melt } \\
\text { Temp } \\
\left({ }^{\circ} \mathrm{C}\right)\end{array}$ & $\begin{array}{l}\text { Amplicon } \\
\text { Size }\end{array}$ & Source \\
\hline \multirow{2}{*}{ Rac1 } & FW: GTGCAAAGTGGTATCCTGAAGT & 55.1 & \multirow{2}{*}{106} & \multirow{2}{*}{ Designed for this study } \\
\hline & REV: AGCTTCTCAATGGTGTCCTTATC & 54.6 & & \\
\hline \multirow{2}{*}{ Kal7 } & FW: GGAGTCAACACGGAGGATAAC & 55.0 & \multirow{2}{*}{159} & \multirow{2}{*}{ Designed for this study } \\
\hline & REV: CTGGAGCAGTTCAGCCATAA & 54.8 & & \\
\hline \multirow{2}{*}{ Disc1 } & FW: TATGCTGCAGGACTACCTACT & 54.8 & \multirow{2}{*}{151} & \multirow{2}{*}{ Designed for this study } \\
\hline & REV: CTGTTCCAGGTCTTCCAATCTC & 55.1 & & \\
\hline \multirow{2}{*}{ Prox1 } & FW: ACGAGTCTGAGGACCAAGAT & 55.1 & \multirow{2}{*}{159} & \multirow{2}{*}{ Designed for this study } \\
\hline & REV: GCTTCCCGAATAAGGAGAGATG & 55.0 & & \\
\hline \multirow{2}{*}{ Gapdh } & FW: AGCTGGTCATCAACGGGAAACC & 59.8 & \multirow{2}{*}{126} & \multirow{2}{*}{$\begin{array}{c}\text { Sourced from } \\
\text { Owczarek et al. } 2015 \\
\text { (Reference 150) }\end{array}$} \\
\hline & REV: CCTTCTCCATGGTGGTGAAGAC & 57.5 & & \\
\hline \multirow{2}{*}{ Hprt } & FW: TCCTCATGGACTGATTATGGACA & 55.3 & \multirow{2}{*}{132} & \multirow{2}{*}{ DJD Lab prior projects } \\
\hline & REV: TAATCCAGCAGGTCAGCAAAGA & 56.3 & & \\
\hline
\end{tabular}

Table 2.4: Primer sequences, melt temperatures, and amplicon size for primer sets used in RT-qPCR. All primer sets aside from reference genes Gapdh and Hprt were designed for this study based on mRNA sequence available from the National Centre for Biotechnology Information (NCBI). 


\subsubsection{Sample Preparation}

Whole hippocampus samples were homogenised (to prevent subsampling error) and a sample was removed and added to $1 \mathrm{ml}$ TRIzol Reagent (ThermoFisher Scientific, \#15596018) for RNA isolation. After incubation at room temperature (RT) for $5 \mathrm{~min}, 0.2 \mathrm{ml}$ chloroform was added to each sample and vortexed to achieve a uniform and opaque pale pink solution. Samples were then centrifuged for $15 \mathrm{~min}\left(4^{\circ} \mathrm{C}, 12,000 \mathrm{~g}\right)$ before removal of the clear aqueous phase to a new microcentrifuge tube. $100 \% \mathrm{EtOH}$ was added to each sample at a ratio of 1:1 and inverted to mix. Samples were then purified using the GeneJET RNA Cleanup and Concentration Micro Kit (ThermoFisher Scientific, K0842). Based on the methods recommended with this kit, a series of wash buffers and centrifuge steps were used. Each sample was placed in a GeneJET Purification Microcolumn and centrifuged for 1 minute (RT, $14,000 \mathrm{~g})$. The supplied wash buffers were then applied to the column in order specified, with 1 minute centrifuge steps between each run (RT, 14,000g). After each wash, elute was discarded. One final centrifuge step with no washing solution was used to completely clear column of excess reagent, before elution of RNA in $20 \mu$ l nuclease-free water in a new vessel.

Following RNA isolation, samples were reverse transcribed to produce cDNA for qPCR analysis. $1 \mu \mathrm{l}$ samples were taken to measure RNA concentration using a NanoDrop ${ }^{\circledR}$ ND-1000 Spectrophotometer (ThermoFisher Scientific). Sample purity was also assessed using recorded absorbances; sample ratios of A260/A230 and A280/260 were compared to expected pure RNA ratios of approximately 2.0 for each. RNA samples were subsequently treated with DNase-I (ThermoFisher Scientific, \#18068015) to ensure no gDNA contamination was present; $2.5 \mu \mathrm{l}$ of both DNase-I (Amplification Grade) and accompanying 10x DNase Reaction buffer were added to RNA, with nuclease free water added to a total volume of $25 \mu$. This reaction was incubated for $10-15 \mathrm{~min}$ (RT) before addition of $2.5 \mu \mathrm{l}$ Ethylenediaminetetraacetic acid (EDTA) and incubation for $10 \mathrm{~min}\left(65^{\circ} \mathrm{C}\right)$ to end inactivate DNase-I.

Samples were then vortexed before a subsample of 5000ng RNA (calculated based on previous absorbances) were taken for reverse transcription, with the remaining RNA being 
stored at $-80^{\circ} \mathrm{C}$. Random hexamers (Integrated DNA Technologies) were added to this sample at a final concentration of $50 \mathrm{ng} / \mu \mathrm{l}$, in addition with $10 \mu \mathrm{M}$ deoxyribonucleotide triphosphate mix (dNTPs) and nuclease free water to a volume of $13 \mu \mathrm{l}$. This reaction mixture was briefly centrifuged, incubated for $5 \mathrm{~min}\left(65^{\circ} \mathrm{C}\right)$, then snap chilled on ice for 3-5 $\mathrm{min}$. $1 \mu \mathrm{l}$ RNase-OUT (Invitrogen, \#10777019), 1 $\mu$ I Superscript III Reverse Transcriptase (Invitrogen, \#18080044), along with accompanying $5 x$ First Strand Buffer and $1 \mu$ dithiothreitol (DTT) were added to the reaction. Again this was briefly centrifuged, and then incubated for 5 min (RT). Samples were then placed in a water bath to incubate for approximately $90 \min \left(50^{\circ} \mathrm{C}\right)$ before inactivation by a final incubation for 15 minutes $\left(70^{\circ} \mathrm{C}\right)$. Samples were stored at $-20^{\circ} \mathrm{C}$ until use.

\subsubsection{Quantitative Polymerase Chain Reaction (qPCR)}

qPCR reactions were prepared in $10 \mu$ l final volumes in duplicate, using a hierarchical system of master-mixing to improve consistency of duplicates and reduce technical error between targets quantifying the same cDNA sample. Each $10 \mu \mathrm{l}$ reaction consisted of $100 \mathrm{ng}$ cDNA, $0.25 \mu \mathrm{M}$ forward and reverse primers for a given pair (Table 2.4), and 5x SolisFast SolisGreen qPCR mix (dnature, \#28-41-00001). The remaining volume consisted of nuclease free water.

qPCR reactions were carried out in a Bio-Rad CFX96TM Real-Time PCR Detection System thermocycler (Bio-Rad, \#185-5096). PCR conditions were set by the following parameters: $95.0^{\circ} \mathrm{C}$ for $2 \mathrm{~min}$ (initialisation), and then 35 repeat cycles of $95.0^{\circ} \mathrm{C}$ for $15 \mathrm{~s}$ (denaturation), $51.0^{\circ} \mathrm{C}$ for $15 \mathrm{~s}$ (annealing), and $72.0^{\circ} \mathrm{C}$ for $10 \mathrm{~s}$ (extension). These were followed by melt curve generation, increasing temperature between $65^{\circ} \mathrm{C}$ to $95^{\circ} \mathrm{C}$ through $0.5^{\circ} \mathrm{C}$ steps for $5 \mathrm{~s}$ each. Melt peaks were generated to detect issues with PCR amplification. For example, multiple peaks may indicate multiple PCR products generated, indicating primers are not specific to the intended target. This could also indicate primer dimerization. 


\subsection{Statistical Analysis}

\subsubsection{General Figure Information}

All figures in this thesis follow the same general colour scheme and layout, to aid understanding of information presented. When comparing poly I:C exposed and salineexposed treatment groups, the colour orange designates the poly I:C group while purple represents saline-group controls. Additionally, analyses based on sex are presented with males in blue and females in pink. When analysed by genotype, data is represented by shade as opposed to colour: WT points are dark grey, while HET points are light grey. Statistical significance markers are also consistent throughout figures. One star $\left({ }^{*}\right)$ indicates a $p$ value less than 0.05 , the marker of classic statistical significance. Two stars $\left({ }^{* *}\right)$ is indicative of a $p$ value less than 0.001 , and three stars $\left({ }^{* *}\right)$ represents a $p$ value less than 0.0001 . 


\subsection{Statistical Analysis: Linear Mixed Models}

\subsubsection{Random and Fixed Effects}

Linear mixed modelling (LMM) of the data presented in this thesis was chosen to account for litter variation as a random but important source of variation. Litter is a factor nested within treatment group, as treatments were applied to dams (i.e. every pup from a given litter has the same treatment, so no litter may contain saline and poly I:C exposed pups). As a pup is not randomly assigned to a treatment group, it is statistically recommended that these are not considered independent variables [177]. LMM provides the option of fitting random variables within a model, accounting for variation within groups of non-independent data (such as pups within a litter).

Using the 'nlme' package on RStudio (Linear and Nonlinear Mixed Effects Models, v3.1153), each measurement of interest was modelled with Litter as a random effect. Each model included the categorical variables Sex, Genotype, and Treatment as main or 'fixed' effects to estimate the effect of each group independently. Each model also includes the full assessment of all two-way and three-way fixed effect interactions, in the following order: order Sex and Genotype, Sex and Treatment, Genotype and Treatment, and finally Sex, Genotype, and Treatment. Model results are presented by Type 1 (sequential) ANOVA. Relationships between groups were further assessed in pairwise-comparisons presented on figures, using Bonferroni's post-hoc test to correct for multiple comparisons. Bonferroni's correction is applied to all relevant statistical tests throughout this thesis to correct for multiple comparisons, chosen due to the robust nature of the test for rejecting false-positive results.

Where pups are grouped across a categorical variable, such as being compared by treatment while not differentiating between sex and genotype (e.g. Figure 5.4), comparisons between groups are carried out using a simplified linear mixed model. This still accounts for litter as a random effect, while only assessing variance associated with the one main effect beng compared. 


\subsubsection{Model Assumptions}

LMM validity requires the following assumptions are met in the data; independence, normality, and homoscedasticity (equal variance of residuals). The independence assumption can be modified to assume independence between nested groups if a random effect is specified; in this model, independence is assumed between litters but not within. Normality and homoscedasticity were assessed through QQ plots, Shapiro's test of normality, and Levene's test for unequal variance in every model. All USV measurement tests met normality and homoscedasticity assumptions, while some RT-qPCR targets did not (see Chapter 5.3). An example of the code used to validate and run these models, developed with guidance from statistical advisor Lisa Woods, is presented in Figure 2.6. 


\section{Figure 2.6: Example RStudio Script of Linear Mixed Modelling}

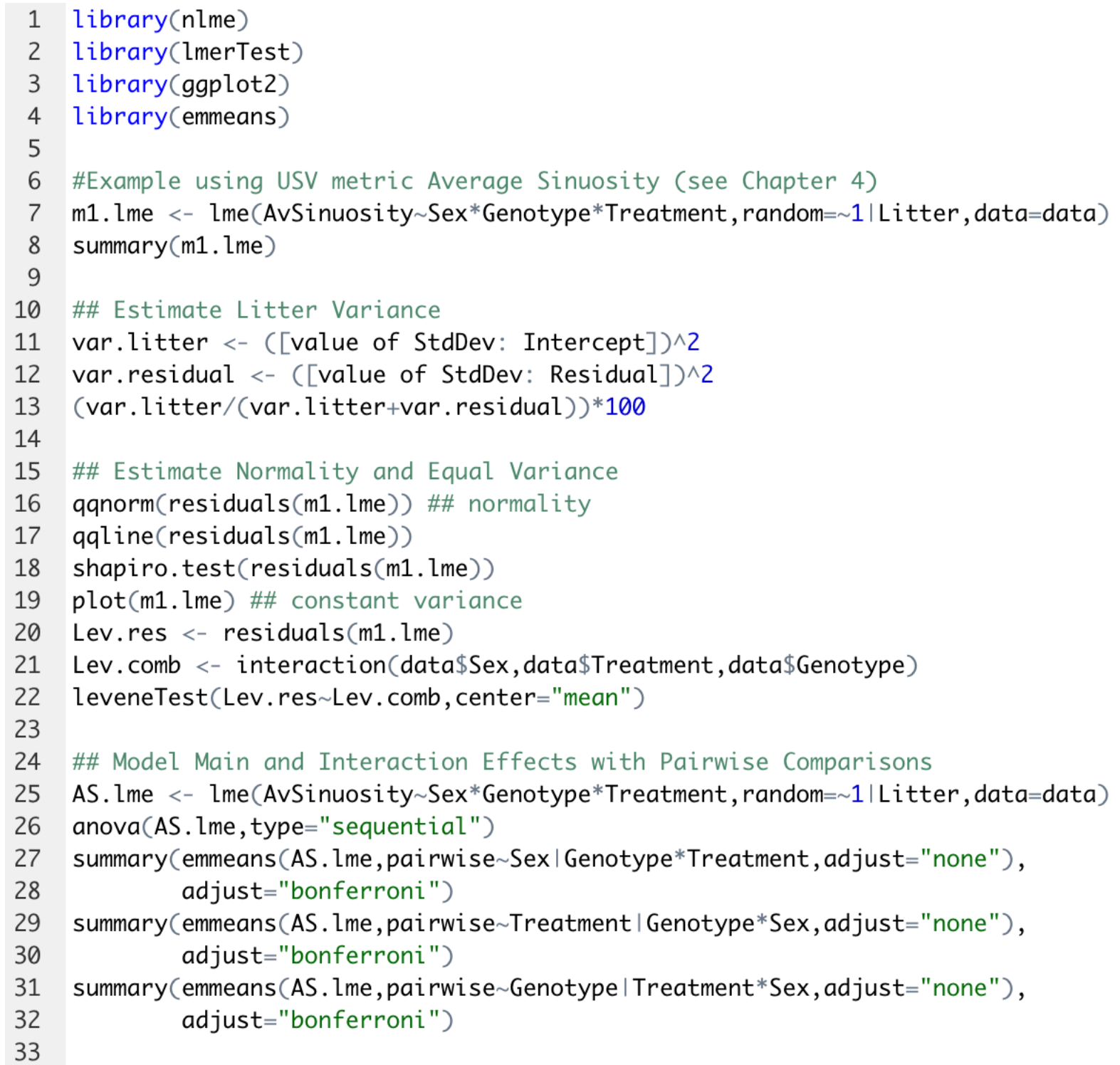

Figure 2.6: Example code used in RStudio to calculate linear mixed models for Average Sinuosity. All call metrics and genes investigated in this study use the same overall process to generate results. 


\section{Chapter 3. Maternal Immune Activation Model}

\subsection{Introduction}

Poly I:C has been used for more than 50 years to elucidate the effects of viral immune responses during pregnancy. These MIA models are flexible, with many variations published that diverge in timing and number of treatments given $[123,125]$. These varying models provide an in-depth view of the complex effects of MIA on offspring development, however risk confusing the research field if appropriate model information is not fully presented. A number of conflicting and irreproducible results have arisen in MIA research as a result of insufficient reporting of model details, which has led to recent reviews working toward guidelines for required information in MIA publications [125, 178].

\subsubsection{Maternal Immune Response}

Poly I:C models have two test groups which require assessment; mothers and offspring. Current literature heavily focuses on the effect of poly I:C exposure on offspring, studying behavioural and developmental alterations as a result of maternal immune response. Interestingly, these models often omit information on the immune response itself. Unvalidated and unequal immune responses are estimated to be one of the biggest sources of variation and conflict between MIA studies [125, 179]. For example, variable molecular weights in commercial batches of poly I:C have been shown to elicit different strengths of immune response $[178,180]$. Without reporting poly I:C weight or quantifying immune response, studies that appear to use comparable models may not be.

Tests such as maternal weight change and temperature increase have been recommended at minimum to address the missing information in many MIA studies [125]. Weight and fever-driven temperature changes are sickness indicators that can be used without excessive time or financial cost. Most temperature measurement techniques are also non-invasive and fast, leading to minimal handling and therefore stress-induced temperature 
change [181]. These include infrared thermometers and cage monitoring systems, while more invasive techniques include methods such as subcutaneous temperature recording chips. Handling stress is a particularly important concern when using pregnant animals, as recent studies have suggested maternal stress may also impact offspring development [182]. Alternative techniques of MIA measurement usually require blood sampling, e.g. proinflammatory cytokine measurement via ELISA. This again increases maternal stress but gives a more informative view of the immune response of each mother.

\subsubsection{Inter- and Intra-Litter Variation}

Litter variation between and within treatment groups is another important consideration in this field. Litter demographic information including litter size and sex ratio is known to affect behaviour and development of pups, however this information is rarely disclosed in published research. Small litter sizes for example are known to affect both physical development and social behaviour, as more attention and nutrition is available to each pup from the mother $[183,184]$. A common finding is that pup weight gain is significantly affected by poly I:C exposure during gestation [185]; this is expected to be a result of affected development in utero, however litter size may also affect this. Litter sex composition may also skew results, particularly in behavioural tests. Sex differences have been identified numerous times across MIA studies [186], and litters that are female-dominated have been shown to present altered behaviour $[187,188]$. Without litter demographics being reported, their effects are unable to be considered.

Within a litter, pups may be too connected to deem independent; animal research guidelines suggest that animals which could not have been assigned to different treatment groups are not independent [177]. This is true of most studies using littermates of multiparous species, but particularly in MIA studies where poly I:C treatment is applied to the mother [189]. Each pup from a poly I:C exposed litter may have been subject to the same immune response during gestation and are therefore closer to technical replicates than independent samples. There is potential for some variation between littermates, as pups have individual placenta and have a fixed position within the rat bicornate uterus [190]. Differences may also 
present between genetically different individuals such as males and females, as commonly reported in NDD research. Statistical analysis often does not reflect the near nonindependence between pups however, potentially leading to misleading $p$ values and conclusions [125].

The aim of this chapter is to provide evidence that maternal immune activation by poly $\mathrm{I}: C$ treatment was successful, and to provide litter demographics to inform assessment of this model. Experimental recommendations from recent guidelines have been used to determine appropriate tests and provide relevant information $[125,178]$. Maternal temperature and weight following treatment will be used to validate MIA, alongside analysis of pup weight as a common metric of altered development. 


\subsection{Maternal Treatments}

\section{Figure 3.1: Maternal Temperature Change Following Treatment}

\section{Maternal Temperature Post-Injection}

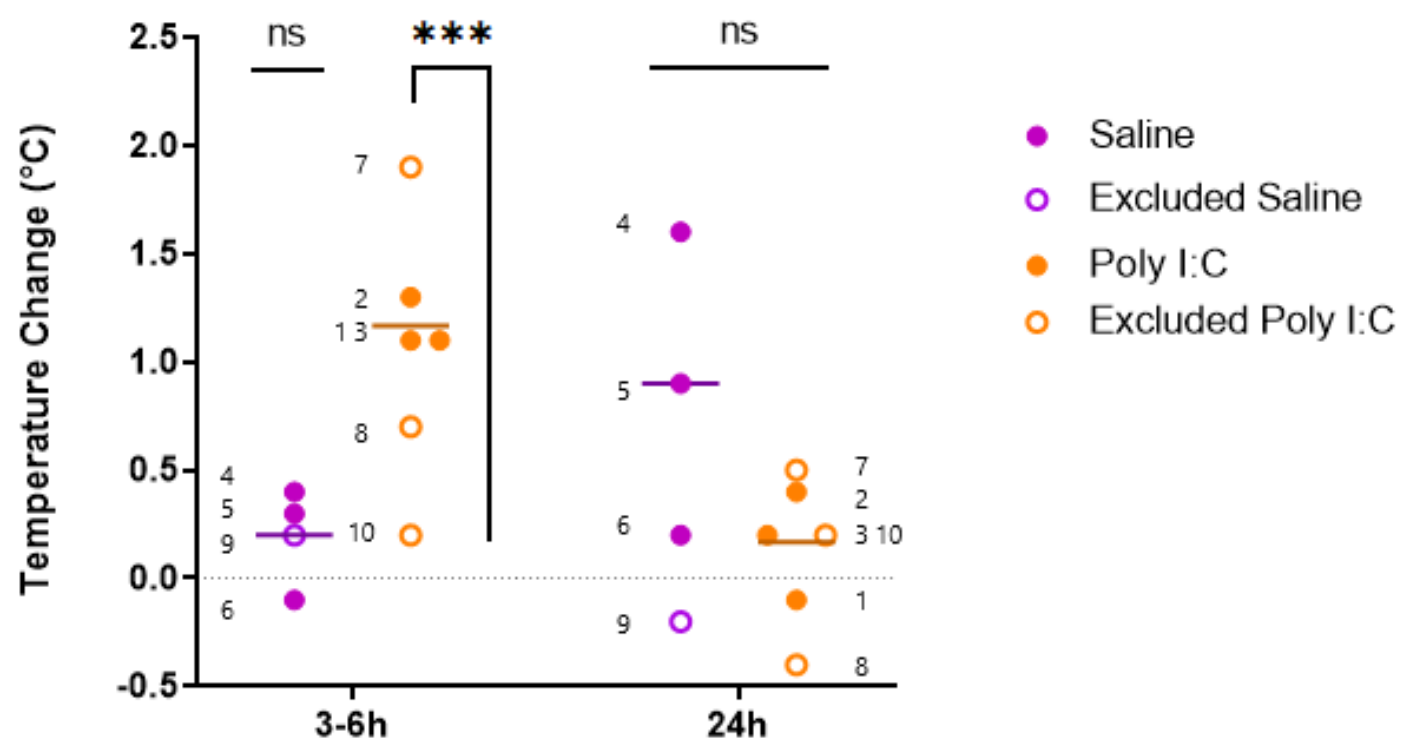

Time

Figure 3.1: Temperature measurements taken 3-6 and 24 hours after subcutaneous injections show immediate temperature increase in poly I:C treated dams but not controls. Original temperature at time 0 (baseline) was subtracted from highest temperature at 3-6 hours and temperature at 24 hours to produce temperature change displayed above. All dams that were treated and produces a litter are shown, with each point numbered with Dam/Litter ID to match paired observations across time points. At 3-6 hours, poly I:C treated dams show a significant temperature increase from baseline $(p=0.0004)$. Poly I:C treated dams and saline dams at both time points do not significantly differ in temperature compared to baseline. 
To verify that an immune had occurred in mother treated with poly I:C, temperature measurements were taken three, six, and 24 hours post-injection. Based on previous studies, it was expected that mothers with a significant immune response would present a feverdriven temperature increase at three to six hours after treatment that returned to baseline measurements after 24 hours. As an immune response is not expected in saline-treated dams, it was predicted that control group maternal temperatures would not vary largely at any time point. Based on the information presented in Figures 3.1-3.3, some dams/litters will be excluded from further study. Maternal information from excluded litters is presented alongside those included and are distinguishable by hollow points as opposed to opaque in this chapter.

Maternal temperature measurements were taken before poly I:C or saline injections, then 3,6 , and 24 hours after injection. Highest temperature at 3-6 hours and 24 hours are shown relative to temperature at time 0 (Figure 3.1). To analyse changes from baseline, twoway paired ANOVA with Bonferroni's correction was used, based on original temperature measurement data at each time point matched across time points within animals. At 3-6 hours, poly I:C treated dams show a strongly significant temperature increase from baseline temperature $(p=0.0004)$. One poly I:C treated dam does not display a large temperature increase as expected, and as a result will be excluded from litter analyses (see discussion). At 24 hours after injection, poly I:C treated dams did not differ significantly from baseline ( $p$ $>0.99$ ). At no point did saline controls significantly differ from baseline, although at 24 hours temperature change almost meets the significance threshold $(3-6 h: p=0.92,24 h: p=0.063)$. Interestingly, saline group dams had a high level of variation, however Levene's test for unequal variances between groups does not meet significance $(p=0.11)$. 


\section{Maternal Weight 24h Post-Injection}

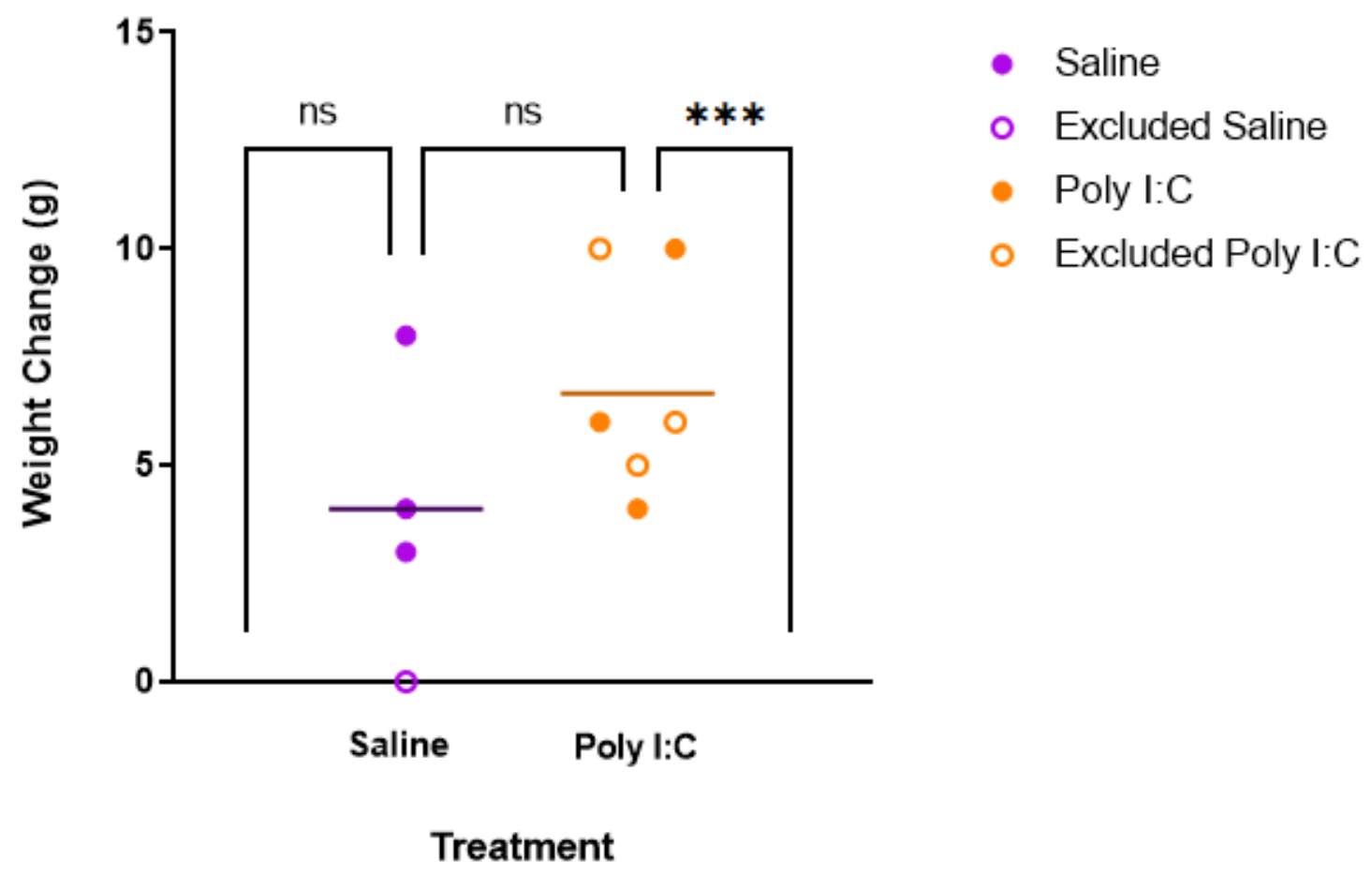

Figure 3.2: Poly I:C treated dams show a significant weight increase from baseline 24 hours after treatment ( $p=0.0008)$, however saline treated dams do not $(p=0.061)$. No significant difference is observed between treatment groups $(p=0.17)$.

Weight change 24 hours after treatment (relative to before injection, time 0) was also assessed in treated and control dams (Figure 3.2). Change from baseline was determined by two-way ANOVA as above, while difference between treatment group was assessed using student's t test of weight change values displayed above. No significant difference is seen between poly I:C and saline dams $(p=0.17)$. Only poly I:C treated dams are statistically higher from baseline weight, however saline treated animals near classical significance (saline: $p=0.061$, poly $1: C: p=0.0008$ ). 


\subsection{Litter Size and Sex Ratios}

\section{Figure 3.3: Litter Demographics}

\section{Litter Composition}

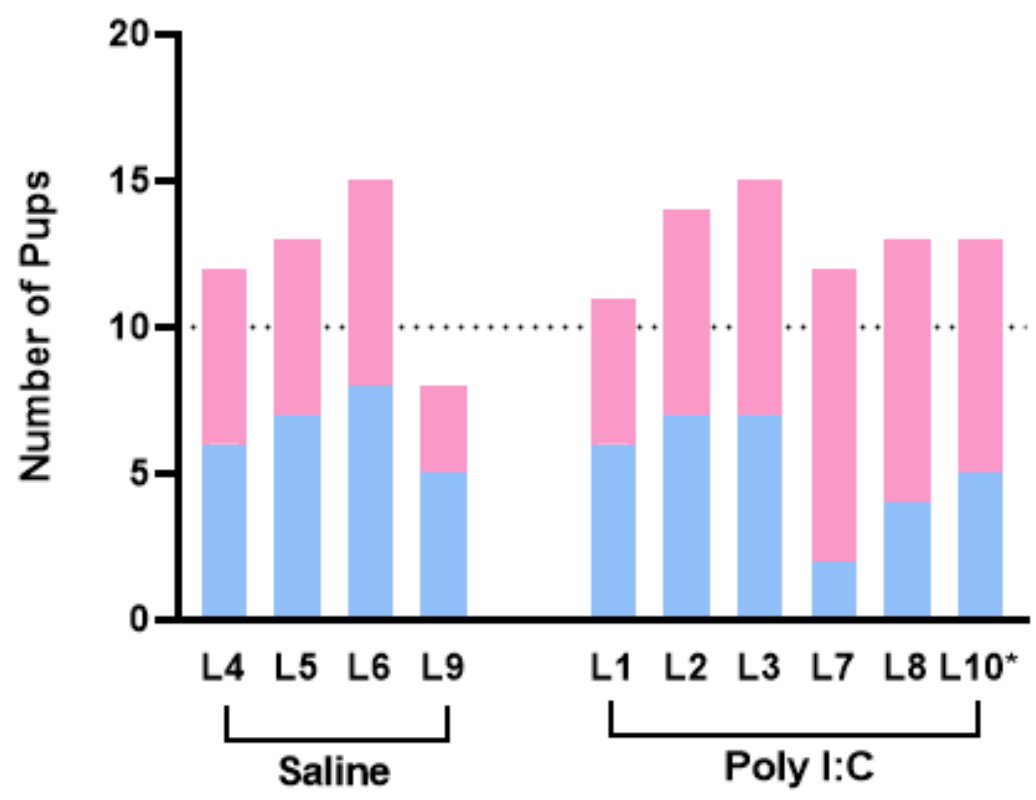

Litter ID

Figure 3.3: Litter size and composition across all 10 available litters. Stacked bars show total litter size at birth, colour differentiated by sex and grouped by treatment. Most litters show an approximately even sex ratio, with the exception of litters 7 and 8 being female dominated. ${ }^{*}=$ Litter 10 information is presented, though this litter was previously excluded (Figure 1).

The 10 litters produced from treated mothers were counted and sexed for further assessment (Figure 3.3). Using a student's t test combining litters within treatment groups, no significant difference is seen in litter size between poly I:C or saline treatment $(p=0.48)$. Additionally, there was only one pre-weaning death (from L6, saline treated), and no litters were identified to have stillbirths. Most litters show a roughly equal sex ratio of male: female pups, however two poly I:C litters have a visually strong bias toward female offspring (L7 and 
L8). Using a two-sided binomial one-proportion z test (recommended to compare an observed proportion with a small sample size to a theoretical proportion of 0.5 ), Litter 7 is significantly skewed toward females $(p=0.039)$ while Litter 8 nears but does not meet classic significance (0.27). Based on the information presented in Figures 1-3, some dams/litters have been excluded from further study. Litter 7 and 8 are excluded based on litter composition (Figure 3.3), Litter 9 for litter size (Figure 3.3), and litter 10 for no maternal temperature change (Figure 3.1). These exclusions will be further elaborated on in the following discussion. Pups from the 6 litters not excluded are presented as the rest of the data in this thesis. 


\subsection{Pup Weight Changes}

Figure 3.4: Treatment Effect on Pup Weight Gain

A

Pup Weight at PND7

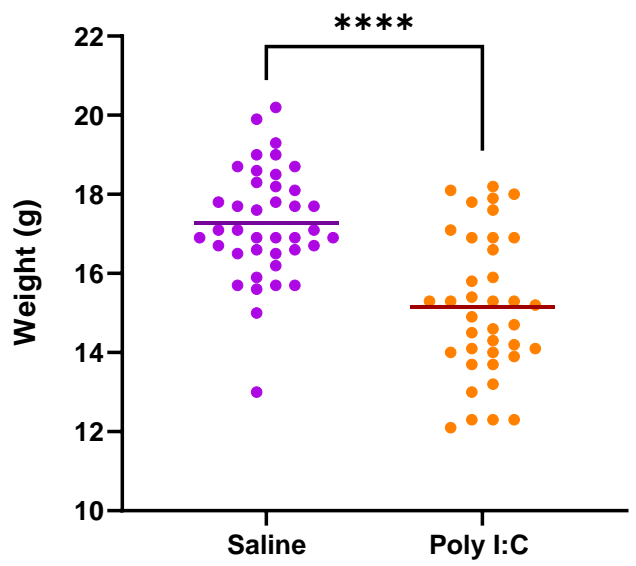

Treatment

C

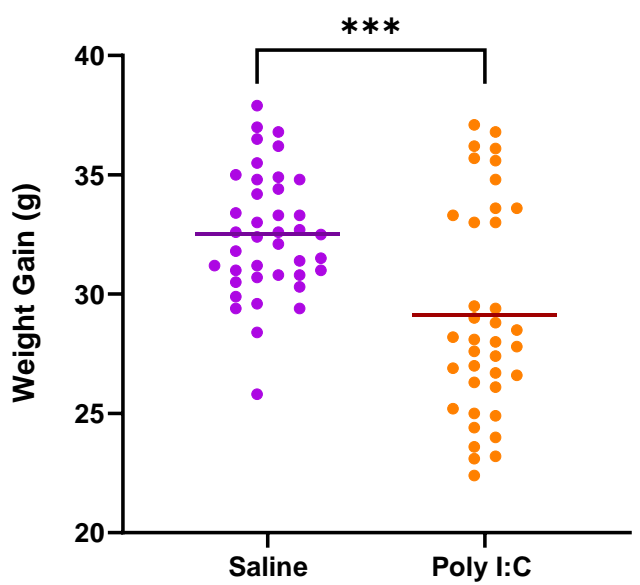

Treatment
B Pup Weight at PND21

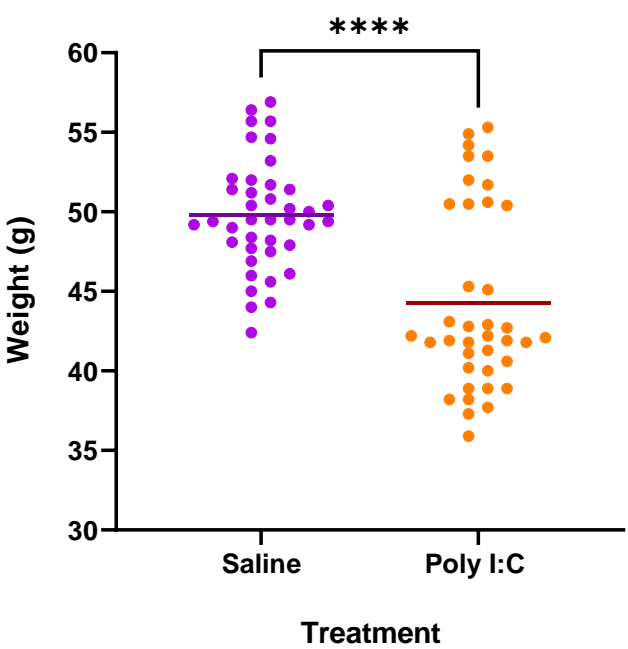

D Percentage Pup Weight Gain

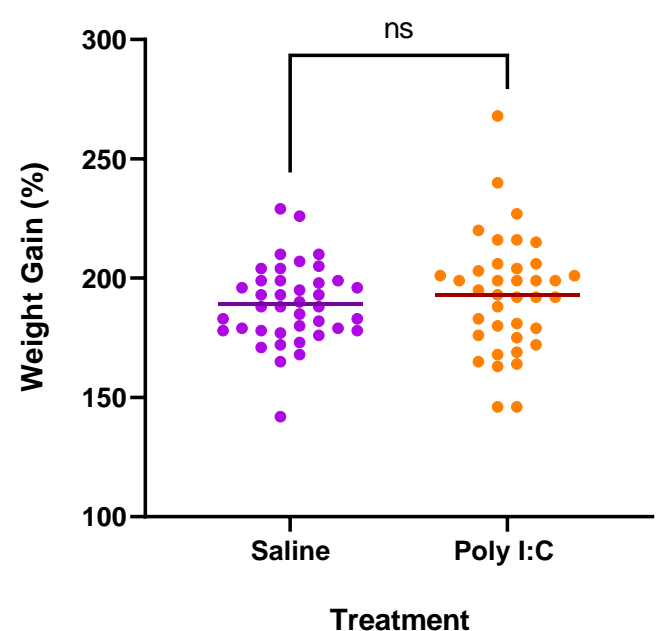

Figure 3.4: Overall pup weight change appears to be affected by treatment but not percentage weight gain. $A / B)$ Weight at PND7 (A) and PND21 (B) is statistically different between treatment groups $(p<0.0001)$. C) Weight change, calculated as the difference between PND21 and PND7, also results in statistical significance between groups $(p=0.0001)$. D) Percentage weight change (calculated as weight change divided by weight at PND7, multiplied by 100) shows all pups roughly double in weight over this time period with no difference between treatment groups ( $p=0.39)$. 
Litter weight was assessed at PND7 and PND21, with pups marked to identify individual weight changes (Figure 3.4). Combining pups from non-excluded litters into treatment groups, it can be seen that poly I:C exposed pups are lighter than saline-exposed pups at PND7 and PND21 (Figure 3.4A-B; students t tests, $p<0.0001$ ). This is carried into pup weight change, calculated as the difference between PND7 and PND21 (Figure 3.4C; students t test, $p=$ 0.0001). This difference is not continued however when accounting for weight gain as a percentage of original pup weight (Figure 3.4D; students t test, $p=0.39$ ). In some instances, weights of poly I:C exposed pups lose an approximately normal distribution and become bimodal (Figure 3.4B-C). This would be consistent with another variable influencing pup size.

\section{Figure 3.5: Pup Weight Gain by Litter Size}

A

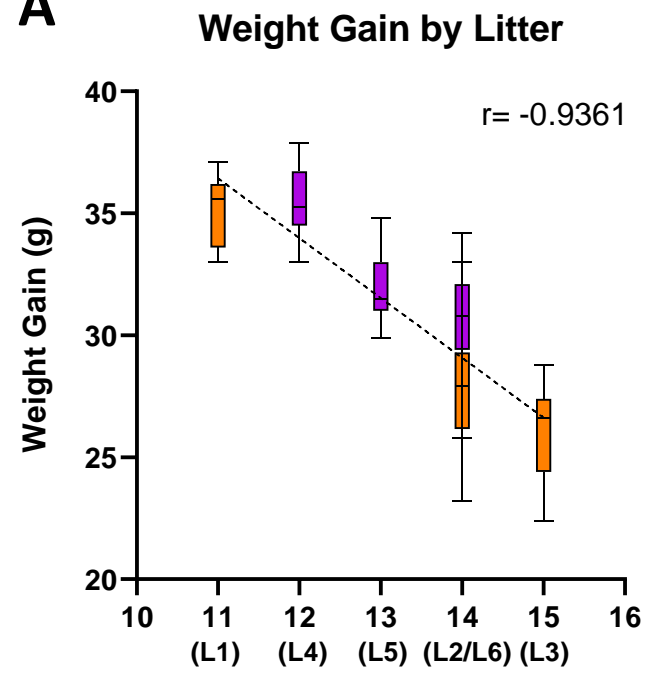

Litter Size (Litter ID)

\section{B Percentage Weight Gain by Litter}

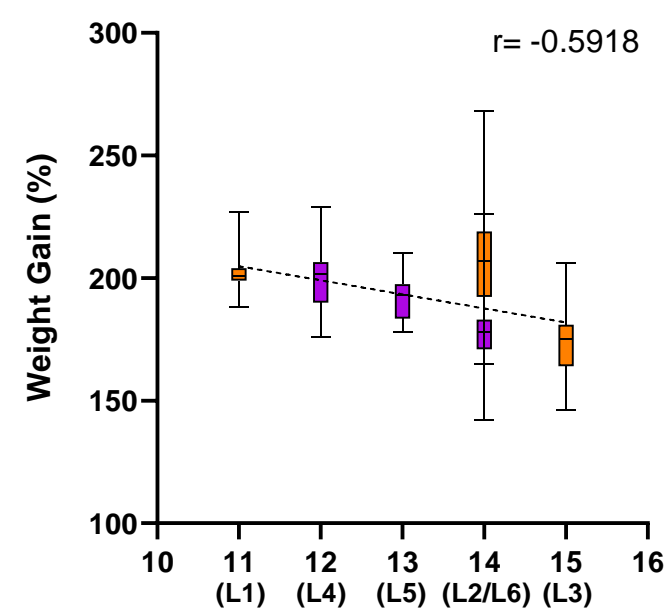

Litter Size (Litter ID)

Figure 3.5: Weight change shows a strong correlation with litter size. Weight change (A) and percentage weight gain (B) data from Figure 10 is presented separated by litter, with litters arranged in size order (size determined by number of pups at day 21). Box plots for each litter are coloured based on treatment as in above figures. A) Weight change shows a strong negative correlation with litter size $\left(r^{2}=0.88, p=0.006\right)$. B) Percentage weight change shows a slight negative correlation with litter size, however this does not reach significance $\left(r^{2}=0.35, p=0.22\right)$. 
To investigate bimodal clustering in Figure 3.4, weight gain in pups was further analysed by individual litter (Figure 5). When litters are organised by litter size, a strong relationship between litter size and pup weight gain is apparent (Figure 3.5A). As litter size increases, pup weight gain decreases $\left(r^{2}=0.88, p=0.006\right)$. This litter dependency is lost when considering percentage weight change (Figure 3.5B) however, with no correlation observed $\left(r^{2}=0.35, p=\right.$ $0.22)$. 


\subsection{Discussion}

\subsubsection{Maternal Immune Response}

Temperature increase and weight change were the two metrics chosen in this research to assess the presence and strength of immune response in mothers treated with poly $\mathrm{I}: \mathrm{C}$ (Figures 3.1-3.2). These results indicate that poly $\mathrm{I}: \mathrm{C}$ treatment does induce a fever response in Wistar SERT +/- pregnant dams, which is present 3-6 hours after injection but reduced to normal at 24 hours. This is consistent with prior studies as discussed previously [185]. Importantly, human epidemiological studies are most closely associated with the development of NDDs in cases where a fever is apparent [107]. While animal models can only mimic human biology to a limited extent, confidence in the relevance of this model to NDD development is only improved by the clear presence of a fever in treatment-group dams.

An unexpected finding from this experiment was the high variance in temperature of saline-treated dams at 24 hours post-injection. This variance is unlikely to be indicative of an immune response from injection, given the lack of response at 3-6 hours. A more likely explanation is this increase being caused by handling stress. For example, the highest temperature at $24 \mathrm{~h}$ was the only measurement taken directly after weekly cage cleaning by animal facility staff. This flaw in the treatment plan resulted in double handling of this dam and a change of environment shortly before measurement, adding stress and likely contributing to the recorded temperature increase. Handling stress is also likely to have had a small effect on measurements during the predicted fever phase, resulting in saline measurements being slightly above baseline as opposed to distributed more evenly above and below. This is unlikely to account for the difference between treatment groups however, as all animals were handled using the same method at the same time points, with no other interruptions.

As a validation test for immune activity, it is important to note that one poly $1: C$ treated dam did not present a large temperature increase (Figure 3.1). This may be an indication that a poor immune response occurred, or simply that this particular dam did not respond with a 
strong fever; individual animals will have slightly different immune responses and of different strengths, consistent with the variation seen between the other poly I:C group dams at 3-6 hours. Other tests to measure immune response, such as a proinflammatory cytokine assay by ELISA, may have provided clearer evidence of immune activation in this individual. This method also more invasive than infrared temperature measurement as blood sampling is required, alongside increased handling time and maternal stress. This likely would have added another significant variable (stress) into the experimental design and confounded temperature measurements, so was not explored. On the basis of this experiment however, this mother and resulting litter were excluded from further analysis as immune response could not be verified.

The results from this study also show that poly I:C treatment does not significantly alter dam weight in this model (Figure 3.2). This does not support common published results, where a halt in weight gain or direct weight loss is often measured post-treatment. There is some evidence to suggest variation in this model however, for example one study reports treatment-correlated weight gain in dams exposed to some concentrations of poly I:C [178]. Additionally, poly I:C treatments have not been well studied in Wistar SERT knockout models, and variation in poly I:C effect between rat strains is well-documented [185].

\subsubsection{Litter Exclusions}

In addition to the dam/litter excluded previously, three more litters were excluded from further study. These exclusions were made based on litter size and sex composition, both of which are known to influence pup behaviour and could potentially confound future results with large litter variations. As mentioned earlier, small litter sizes have been shown to alter pup behaviour especially in early life as more maternal attention is available for each pup. Fewer pups in a litter also allows each pup to receive more nutrition from the mother, reducing competition for space during feeding times and potentially improving physical growth and development. For this reason, Litter 9 was excluded from further analysis as it is far smaller than other litters with only 8 pups (Figure 3.3). Sex ratios within a litter have also been identified in previous work as an important behavioural factor [188]. To prevent female- 
dominated litters skewing results from any behavioural studies carried out, Litter 7 and 8 were also excluded from future results (Figure 3.3). While only Litter 7 contained a statistically significant high proportion of females, the visible skewing of Litter 8 also raised concern that the high number of females would impact maternal and pup behaviour so both were excluded.

It should be noted that in accordance with ethics guidelines, mothers post-weaning from litters and litters that were excluded from future study were still used where possible. This includes being transferred to other projects for training or creating a brain tissue bank for methods testing and pilot research for future projects.

\subsubsection{Pup Weights}

In numerous studies, pup weight is shown to be smaller in poly I:C exposed litters than controls. This finding was originally supported by the data presented in this thesis; when grouped by treatment, pups exposed to poly I:C show reduced weight directly as well as weight change (Figure 3.4). Percentage weight change does not differ between groups however, indicating that pups which are smaller at PND7 remain smaller but gain the same percentage of body weight as larger pups. Additionally, the appearance of two clusters within the poly I:C data suggested another variable may be influencing weight change measurements. Considering the previously mentioned evidence that litter size may affect physical development of pups, data was further analysed by litter. This revealed a strong, significant correlation between litter size and weight change (Figure 3.5A). The bimodal distribution of poly I:C group pups seen previously is clearly differentiated by litter; the smallest litter (L1) forming the highest weight group, and the two largest litters (L2 and L3) forming the bulk of the treatment group.

Percentage weight change, which appeared unaffected by treatment group, is also unaffected by litter size (Figure 3.5B). This is consistent with the earlier discussion of litter size; pups from larger litters are small in early life and receive enough nutrition to grow, but not reach or overtake the weight of larger pups from smaller litters. It is important that no 
difference in percentage weight gain is not conflated with no differences in overall weight gain. This difference is clearer when considering the difference in means of PND7 vs PND21 pups (Figure 3.4): group means differ by approximately 2 grams at PND7, have the same mean percentage growth, and differ by approximately 6 grams at PND21. A small pup and a large pup may both show a weight increase of $200 \%$, however the difference between their raw weight measurement has grown.

Due to litter size having such a strong effect, it is difficult to ascertain whether poly I:C exposure had an effect on pup weight compared to controls. Considering percentage weight change does not decrease in treatment group pups, this suggests that the ability to gain wait is not affected by MIA. Where altered pup weights are reported in the literature however, it is usually through direct weight comparisons or weight change and not percentage weight change. Interestingly, core papers which report reduced weight gain in poly I:C exposed pups do not always report litter size. Studies lacking litter-based information such as size and sex ratio may be presenting results confounded by these factors, making it difficult to assess the validity of those findings; this is further exemplified by these results, where litter size has a significant effect. A lack of demographic and validation information is a common issue in MIA research, as mentioned earlier.

The results in this chapter were generated to validate and improve understanding of the natural variation in this model, according to recent recommendations and reviews [125, 178]. While this evidence does not support maternal weight and pup weight alterations, it does show a fever-driven temperature change in poly I:C group dams. These results have also highlighted the importance of validation tests and litter-based assessment in MIA models. Variance between individual animals has confounded results in this research alone and has likely led to misinterpretation of some results published in current literature [189]. Knowing the effect of litter is likely contributing to intra- and inter-treatment variation, all further statistical analysis will account for litter as random variance or consider litter number as total sample size. This follows recent guidelines for statistical analysis of MIA models and improves the likelihood that the results will be reproducible. 


\section{Chapter 4: Behavioural Study; Maternal Separations}

\section{1: Introduction}

\subsubsection{Ultrasonic Vocalisations in Pup Behavioural Research}

Ultrasonic vocalisations (USVs) are a unique and complex method of communication in rodents. Rats, mice, and other laboratory animals were discovered to produce ultrasonic sounds, or 'calls', in 1954 and have been used since as a measurement of social behaviour [191]. USVs are produced in a wide variety of ages and situations including social play, pain/stress, aggression, and pup/mother communication. Each situation is isolated to a specific call frequency band usually ranging between $20-60 \mathrm{kHz}$ and can be identified and analysed by spectrograph [192]. Differences in rate of calling and call complexity are common metrics used to measure communicative and behavioural differences between test groups, often correlated with physical behaviour to provide a more comprehensive understanding of social communication [193]. The potential complexity of USV studies make them a powerful tool validating and investigating rodent models of human conditions characterised by altered behaviour.

Maternal separation USV studies are one of the most common measure of behaviour in young pups. Pups use USVs primarily to bond and communicate needs to their mother, a social behaviour shared between species which raise live young [194]. When separated from the mother, pups show altered calling behaviour potentially driven by stress, retrieval request, or response to cold $[195,196]$. Recording calls during the maternal separation period allows investigators to assess differences in pup stress response without causing physical harm. A limitation in this method is that physical responses can rarely be correlated with call types as young pups have limited physical movement and are blind until approximately PND13; the one physical behaviour available is righting reflex [197]. This can make it difficult to judge whether novel calling differences between groups have a relevant meaning to social communication, however this may be improved by relating pup USV to further behavioural tests later in life. Call alterations that have been consistently linked to increased pup stress 
include a change in call number and increased shape complexity e.g. multiple frequency changes within a single call [198]. These are often the primary considerations in pup USV studies and will be assessed here along with other metrics that are less commonly reported (Table 1).

Abnormal behaviour in SERT knockout models and poly I:C induced MIA models have been studied repeatedly in current literature, however not in combination. Adult SERT knockout mice have shown increased reward seeking behaviour, increased fear response, and reduced sexual activity in adult males $[182,199,200]$. Altered USV calling is also seen in adults, with more complex multi-part calls during social interaction and reduced $50 \mathrm{kHz}$ exploratory calling [201]. Few studies have been done in SERT pups specifically; one shows time calling alteration in SERT WT but not HET pups following SSRI exposure [202], and a related study uses a Tph2 knockout model to show reduced number of calls in pups lacking brain serotonin [203]. MIA studies typically show altered calling between treatment and control groups. For example, one comprehensive study reports differences in number of calls, time calling, and call shapes between poly I:C and saline exposed litters aged PND6-14 [193]. Based on our current understanding of these models separately, it is predicted that pups in this study will show altered calling between treatment groups, with potentially stronger changes in SERT HET pups compared to WT.

\subsubsection{USV Measurement and Classification Tools}

Advances in spectrographic representation and recognition of calls have allowed increasingly thorough analysis of USV composition, with machine learning becoming a strong classification tool. Audio visualising programs initially developed for other purposes, such as Raven for analysing bird calls [204], provided an early wave and still-utilised method of USV analysis. These programs provide the ability to measure calls in a number of ways but require full manual classification of each call. Newer technologies utilise machine learning and aim to automate the classification process, reducing the time cost on researchers and individual subjectivity [205]. Current methods however are prone to false negatives and less accurate results, usually attributable to under-trained networks. 
This research utilises the machine learning MATLAB program DeepSqueak and extension fork DeepSqueak Screener. DeepSqueak is designed to improve rodent USV studies by using trained neural networks to recognise calls and automatically highlight them from background noise [206]. Detected calls can be manually reviewed and accepted, rejected, or slightly altered; false negatives cannot be assessed however, as the DeepSqueak program fractures audio files as part of the detection process. The fork DeepSqueak Screener allows for repairing and viewing the spectrogram of the entire detection file, so users may manually add calls missed by the software. Detection files can then be used to export call statistics, providing precise measurements for each call (table 1). Only some are used in this research, and reasoning for this will be elaborated on in the discussion.

The aim of this chapter is to establish whether behavioural changes can be detected in PND7 pups exposed to poly I:C during gestation. Based on previous published research, changes in pup USVs such as number, length, and complexity of calls may be altered following MIA. SERT genotype may also impact behaviour or alter the effect of MIA, the hypothesis this research is designed to investigate. We predict treatment differences will be present in poly I:C exposed pup USVs, and these differences will be amplified in SERT HET pups consistent with a model of increased susceptibility to MIA during gestation. 
Table 4.1: List of Potential and Investigated DeepSqueak Call Metrics

\begin{tabular}{|l|l|}
\hline Measurement & Description \\
\hline Number of Calls & Total number of calls in each audio file \\
\hline Time Calling (s) & Addition of all linear call lengths in an audio file \\
\hline Average Call Length (s) & Mean of all call lengths \\
\hline $\begin{array}{l}\text { Average Principal Frequency } \\
\text { (kHz) }\end{array}$ & Mean of all mean call frequencies \\
\hline $\begin{array}{l}\text { Average Change in Frequency } \\
\text { (kHz) }\end{array}$ & $\begin{array}{l}\text { Mean of every call minimum frequency subtracted from } \\
\text { maximum frequency }\end{array}$ \\
\hline Power (dB/Hz) & Strength of call signal, calculated as amplitude/frequency \\
\hline Slope (kHz/s) & Height of call/length of call \\
\hline Average Sinuosity (SI) & $\begin{array}{l}\text { The 'curviness' of each call averaged; calculated by total } \\
\text { length of call contour/linear call length and reported as } \\
\text { Sinuosity Index (SI) }\end{array}$ \\
\hline Average Tonality & $\begin{array}{l}\text { The recognisability of each call from background noise; } \\
\text { calculated as 1-(geometric mean power/arithmetic mean } \\
\text { power) averaged }\end{array}$ \\
\hline
\end{tabular}

Table 4.1: A list of potential statistics generated through DeepSqueak, alongside a description of how they are calculated. Grey-shaded rows were available to use but not analysed in this study. 


\section{2: Results}

Table 4.2: Overall Effects on USV Metrics

\begin{tabular}{|c|c|c|c|c|c|c|c|c|}
\hline & \multicolumn{7}{|c|}{ Main and Interaction Effects } \\
\hline & & $\operatorname{Sex}(S)$ & $\begin{array}{c}\text { Genotype } \\
\text { (G) }\end{array}$ & $\begin{array}{c}\text { Treatment } \\
\text { (T) }\end{array}$ & $S^{*} G$ & $\mathrm{~S}^{*} \mathrm{~T}$ & $\mathrm{G} * \mathrm{~T}$ & $\mathrm{~S} * \mathrm{G} * \mathrm{~T}$ \\
\hline \multirow{6}{*}{ 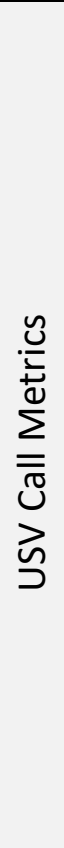 } & Number of Calls & 0.58 & 0.0094 & 0.26 & 0.16 & 0.60 & 0.43 & 0.11 \\
\hline & Time Calling & 0.20 & 0.033 & 0.36 & 0.75 & 0.76 & 0.82 & 0.45 \\
\hline & $\begin{array}{l}\text { Average Call } \\
\text { Length }\end{array}$ & 0.33 & 0.99 & 0.72 & 0.24 & 0.47 & 0.35 & 0.66 \\
\hline & $\begin{array}{l}\text { Average Principal } \\
\text { Frequency }\end{array}$ & 0.83 & 0.0026 & 0.83 & 0.57 & 0.96 & 0.95 & 0.50 \\
\hline & $\begin{array}{l}\text { Average Frequency } \\
\text { Change }\end{array}$ & 0.43 & 0.0004 & 0.14 & 0.26 & 0.80 & 0.31 & 0.67 \\
\hline & Average Sinuosity & 0.55 & 0.0003 & 0.12 & 0.10 & 0.55 & 0.041 & 0.26 \\
\hline
\end{tabular}

Table 4.2: P values generated by linear mixed modelling for each call metric. Significant values are highlighted in red $(p<0.05)$, while those nearing significance are shown in yellow $(p<$ 0.20). All $p$ values are reported to 2 significant figures, up to 4 decimal places. All metrics tested produced the same degrees of freedom (Treatment: numerator degrees of freedom $=1$, denominator degrees of freedom $=4$; All other models: numerator degrees of freedom $=1$, denominator degrees of freedom $=57)$, with $F$ statistics for each presented in Appendix 2. All models meet necessary assumptions of normality and equal variance as tested by Shapiro's and Levene's test (data not shown). 
Linear mixed modelling (LMM) was carried out on pup USV measurements with Litter ID accounted for as a random effect. Assessing main and fixed effects in the order displayed above, LMM for each metric was carried out through type 1 sequential ANOVA (Table 2). Genotype as a main effect was the strongest across metrics, with all except Average Call Length reaching classic statistical significance. By contrast, treatment effect does not reach this threshold across any metric. Average Frequency Change and Average Sinuosity provide the strongest treatment effects ( $p=0.14, p=0.12$ respectively), nearing significance. Sex effect is relatively weak across all metrics, however shows stronger but not statistically significant results when interacting with Genotype effect. Of all metrics assessed, Average Sinuosity presents the statistically strongest results across main and interaction effects. This includes a significant Genotype and Treatment interaction, suggesting treatment differences may be modulated by genotype.

Firstly, pups were clustered by sex and genotype to assess differences between treatment groups (Figure 4.1). Average Sinuosity was found to differ significantly by treatment in male SERT HETs $(p=0.038)$. No other differences across sex/genotype groups and metrics met classic statistical significance. Treatment differences across all metrics however were nearest to significance when comparing male SERT HETs, with the exception of Time Calling (Number of Calls: $p=0.094$, Call Length: $p=0.28$, Average Principal Frequency $p=0.63$, Average Frequency Change $p=0.17)$. This trend is also represented visually, with HET pairs often showing visually larger differences compared to WT (e.g. Figure 4.1A, C).

Figure 4.1: Treatment differences in six USV metrics with pups grouped by sex $(M=$ male, $F=$ female $)$ and genotype (WT= SERT wildtype, HET=SERT heterozygous). Pairwise comparisons of estimated marginal means were derived from $L M M$ for each metric. Significant differences between treatment and control male SERT HET pups in call sinuosity (F) were the only comparison that met statistical significance ( $p=0.038)$. 
Figure 4.1: Treatment Comparisons Across All Groups

A

Number of Calls

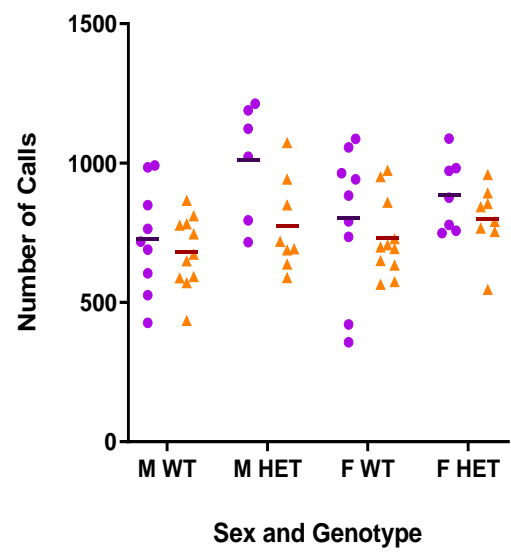

C

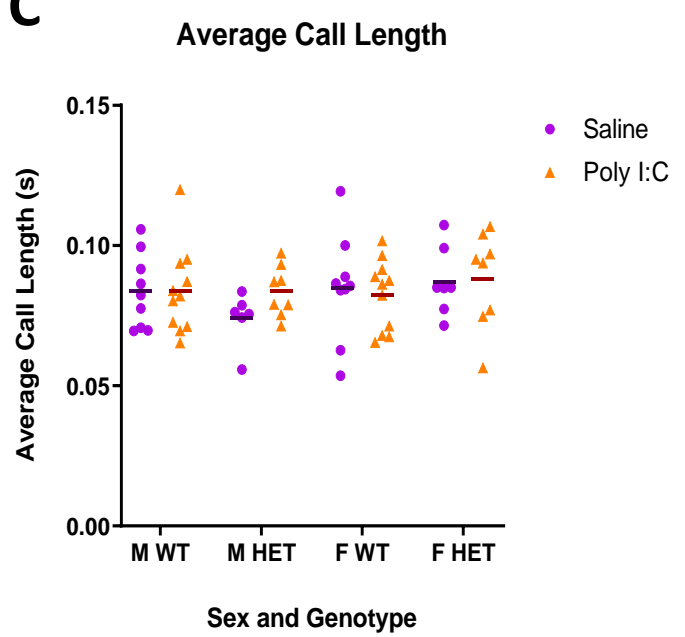

E

Average Frequency Change
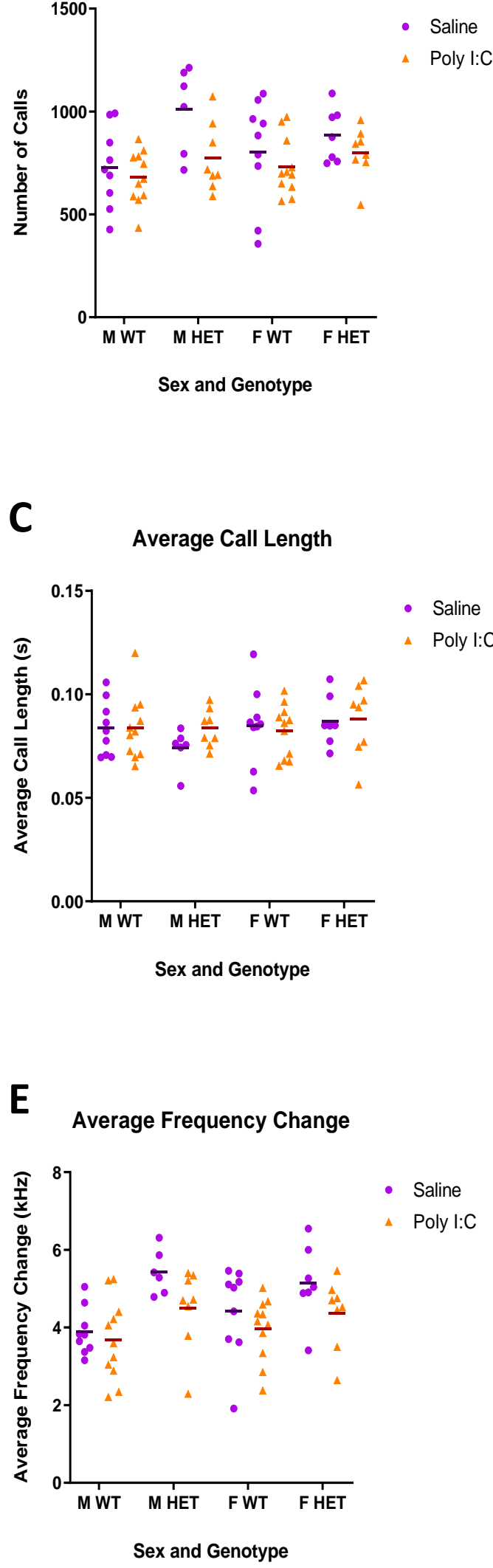

B

D Average Frequency
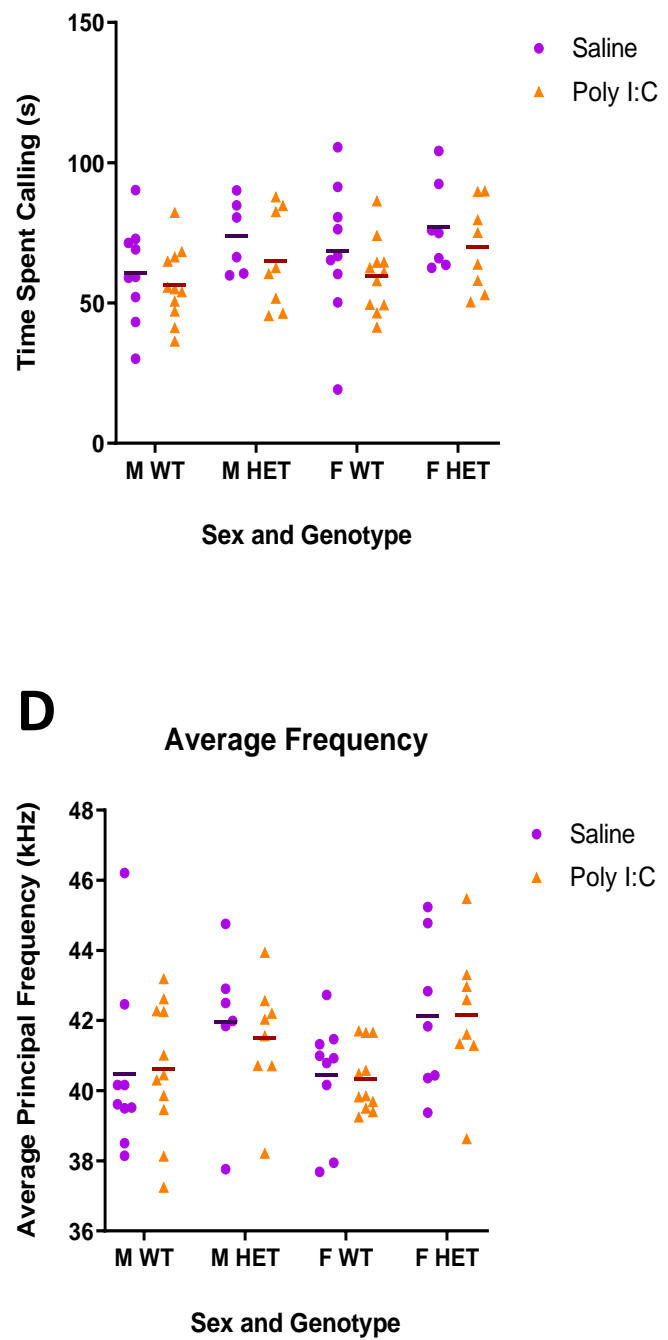

$\mathbf{F}$

Average Sinuosity

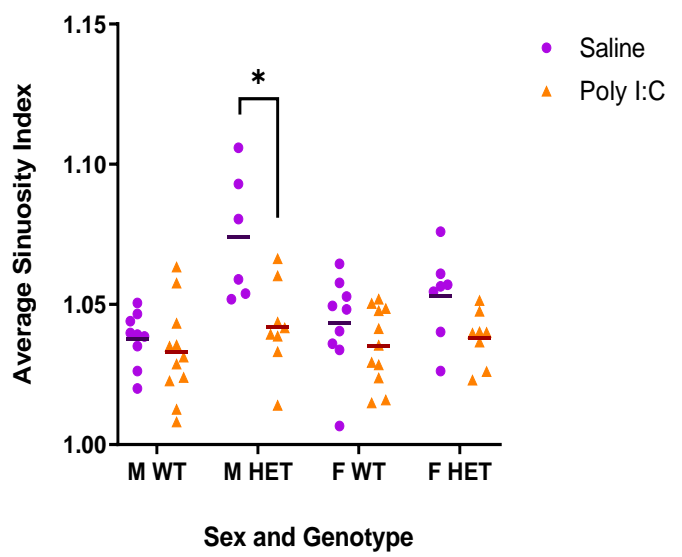


Figure 4.2: Significant Sex and Genotype Comparisons Across All Groups

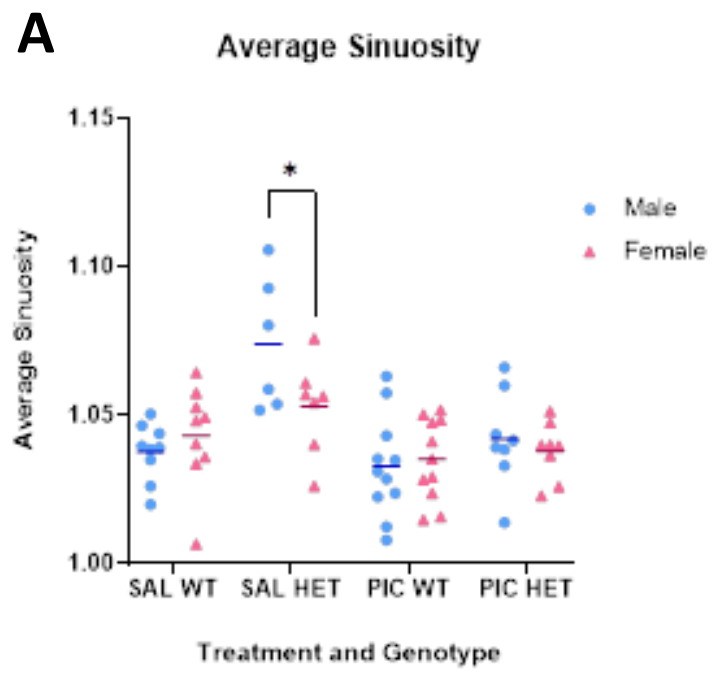

B

Number of Calls

C

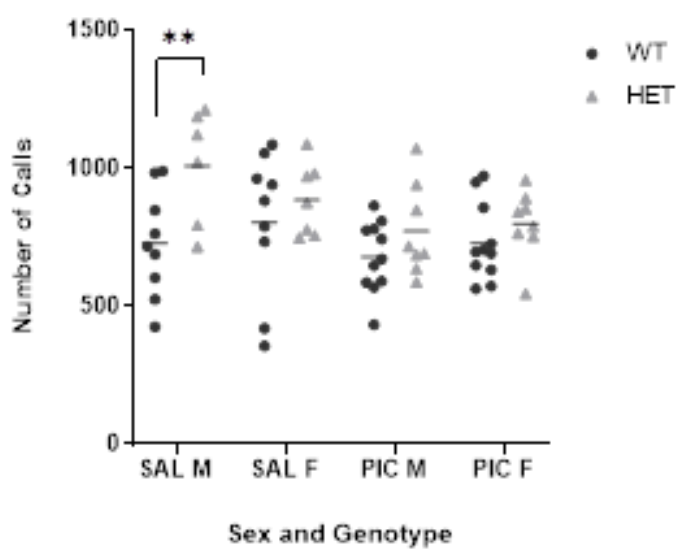

D

Average Frequency Change

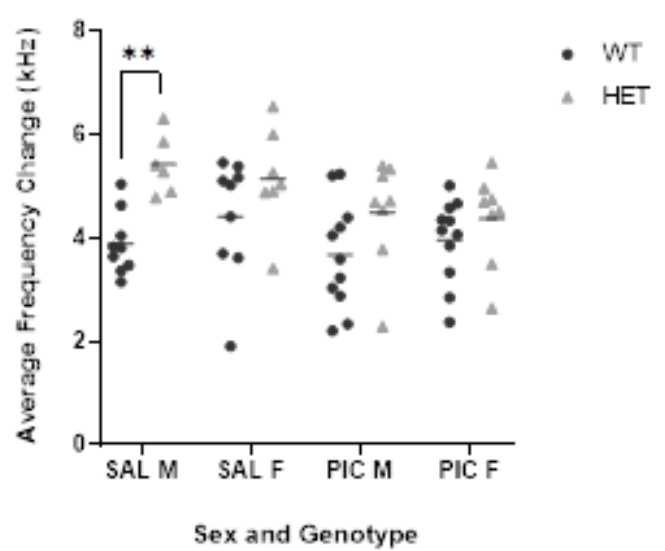

$\mathbf{E}$

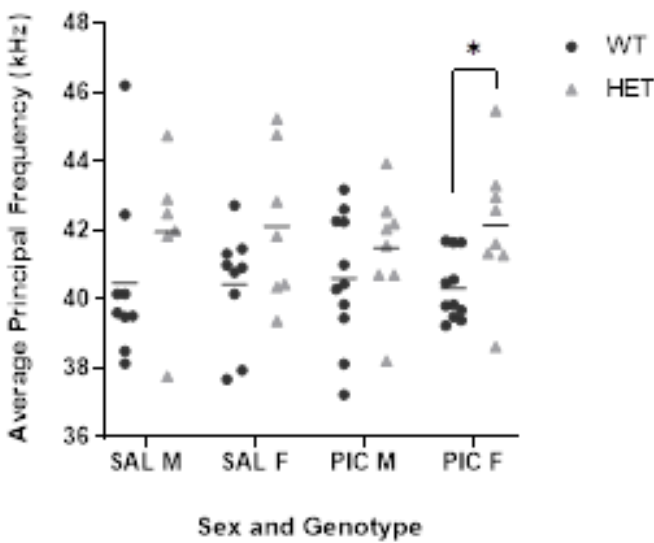

Average Sinuosity

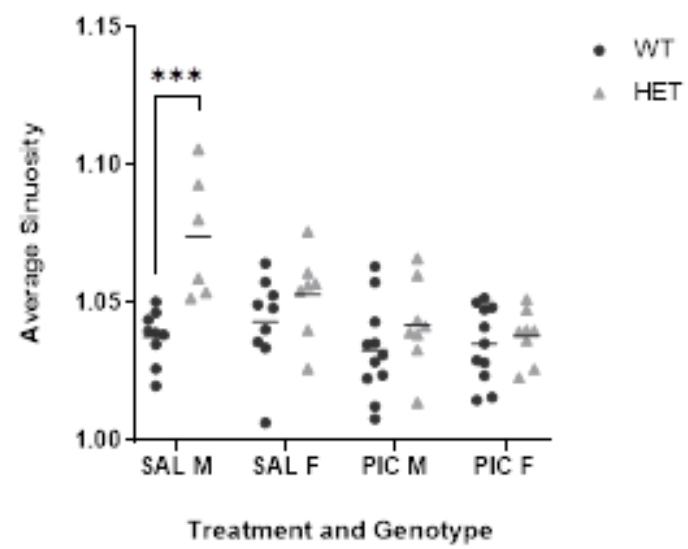


After assessing pairwise differences in Treatment, Sex and Genotype pairings were compared (Figure 4.2). Only one significant sex difference was observed, between male and female saline group HETS ( $p=0.037$ ) in Average Sinuosity (Figure 4.2A). Number of Calls and Average Call Length also near but do not reach significance when comparing saline group HETs, with $p$ values of 0.090 and 0.10 respectively (Appendix 3 ). In contrast, $4 / 6$ metrics show significant pairwise genotype comparisons; this reflects the common finding of a significant genotype main effect (Table 4.2). Number of Calls, Average Frequency Change, and Average Sinuosity all show significant pairwise differences in saline males across genotype (Figure 4.2B, D, E). Average Principal Frequency nears but does not show a significant difference in this pair $(p=0.12)$, instead presenting a significant difference $(p=0.028)$ between genotypes in poly I:C exposed females (Figure 4.2C). In all four metrics with significant genotype differences, the overall trend of genotype is conserved where HETS visually appear higher than males across all pairs. Time Calling also reflects this trend, while Average Call Length does not (Appendix 3).

Figure 4.2: All significant pairwise differences are displayed between Sex (A) and Genotype (B-E) groups. All $p$ values were produced by pairwise comparisons of estimated marginal means (EMMs) based on full linear mixed modelling. Non-significant comparisons for all other call metrics can be found in Appendix 3. A) Pups are grouped by treatment and genotype, compared between sexes (male= blue, female $=$ pink) for Average Sinuosity. Only one comparison yields a significant finding, with saline-group HET males statistically significantly higher than equivalent females $(p=0.037)$. B-E) Pups are grouped by treatment and sex, compared by genotype (light grey= SERT HET, dark grey=SERT WT). Number of Calls, Average Frequency Change, and Average Sinuosity $(B, D, E)$ all present a statistically significant increase in salinegroup male HETS compared to saline-group male WT ( $B, p=0.0022 ; D, p=0.0036 ; E, p=0.0001$ ). Average Principal Frequency (C) also shows a statistically significant increase in SERT HETS, however only when comparing poly I:C exposed females ( $p=0.028)$. 


\section{Figure 4.3: Litter Variation Within Metrics}

A

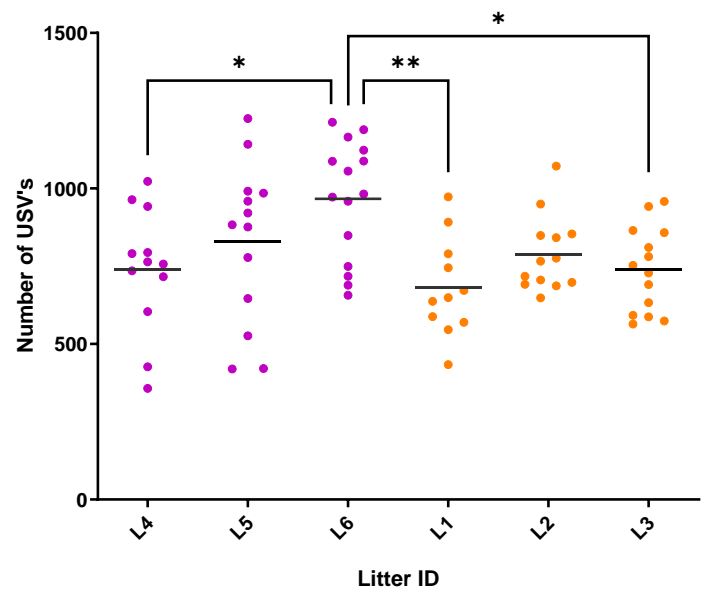

C

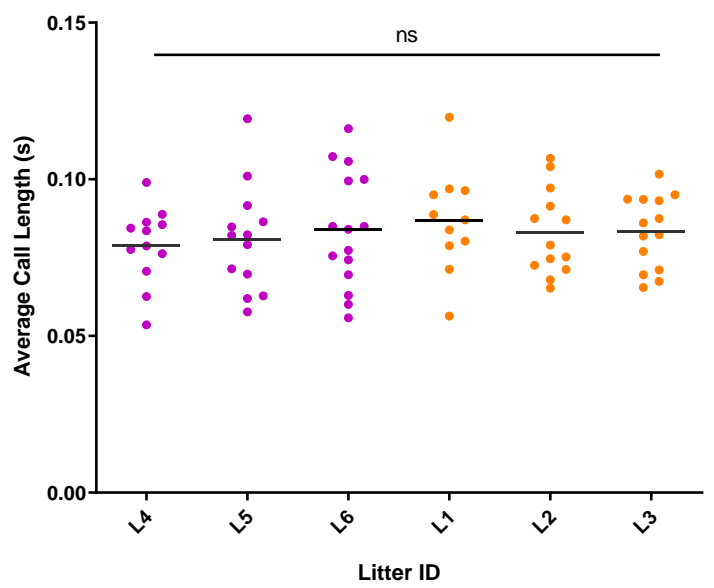

E

Average Frequency Change

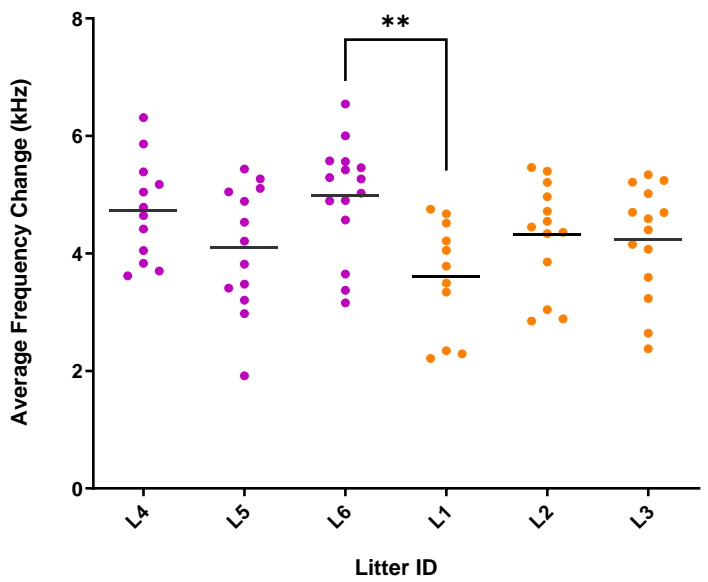

B

Total Time Calling

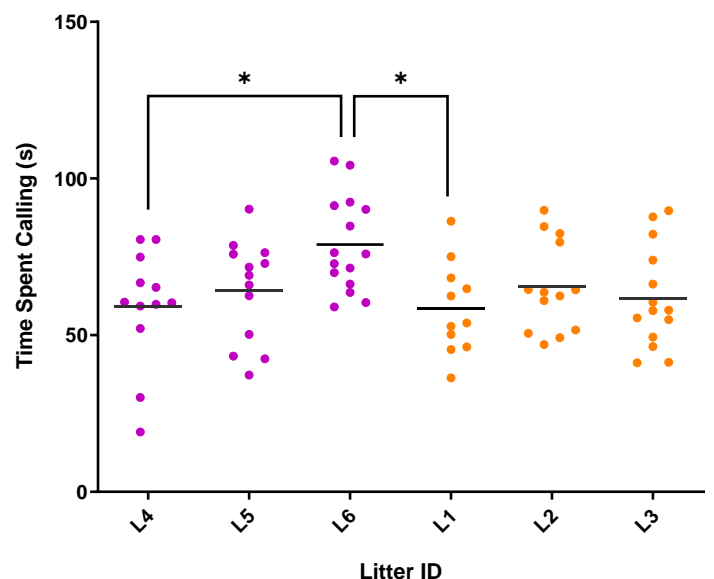

D Average Principal Frequency

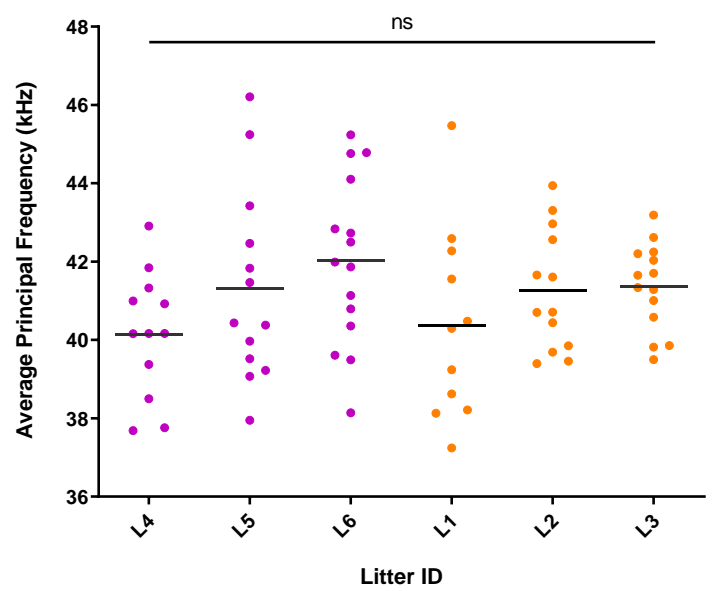

$\mathbf{F}$ Average Sinuosity

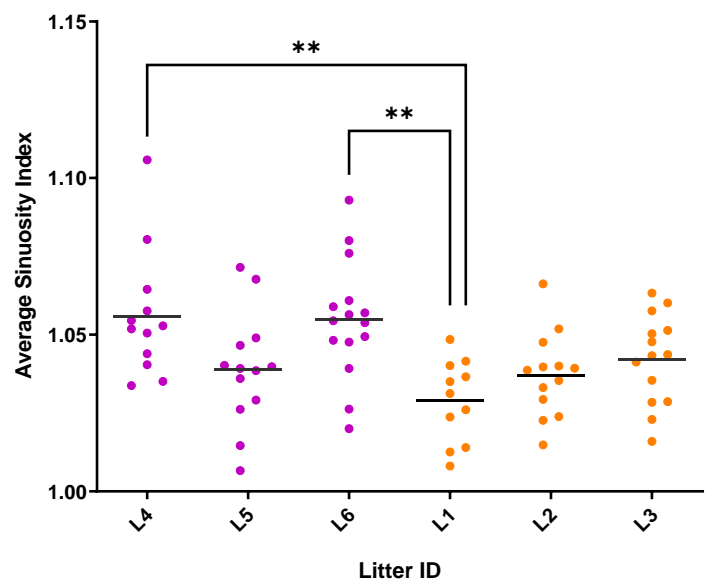


Based on the strong effects of litter variation in physical pup development (see Chapter 3), litter variation was assessed in all six call metrics (Figure 4.3). Two metrics, Average Call Length and Average Principal Frequency, do not show any statistically significant litter differences (Figure 4.3C/D). In the other four metrics, significant differences are observed. Litter 6 significantly differs from Litter 1 across all four metrics, potentially driven by biological and/or treatment variance. Significant differences across treatment groups also include Litter 1 differing from Litter 4 in Average Sinuosity (Figure 4.3F, p=0.0033) and Litter 6 differing from litter 3 in Number of Calls (Figure 4.3A, $p=0.0192$ ). Final significant differences between litters exist within treatment groups; specifically, Litter 6 and Litter 4 differ significantly in Number of Calls and Time Calling (Figure 4.3A, $p=0.0308$ and Figure 4.3B, $p=0.0282$ respectively).

Figure 4.3: Pups are grouped by litter to assess variation across all six call metrics. One-way ANOVAs were used to assess variation between litters, with Bonferroni's correction for multiple comparisons used as a post-hoc test. Litters are coloured by treatment for additional context, with poly I:C exposed litters in orange and saline controls in purple as seen previously. A) Litter 6 shows significant differences in Number of Calls between poly I:C exposed litters $1(p=0.0030)$ and $3(p=0.0192)$, as well as saline exposed Litter 4 ( $(p=0.0308)$. B) Litter 6 again significantly differs from Litter $1(p=0.0251)$ and Litter $4(p=0.0282)$ in Time Calling. $C / D)$ No significant differences are seen across litters. $E)$ Average Frequency Change shows significant litter variation between Litters 1 and $6(p=0.0080)$. F) Average Sinuosity also presents a significant difference between Litters 1 and $6(p=0.0031)$, as well as Litters 1 and 4 ( $p=0.0033)$. 


\subsection{Discussion}

\subsubsection{Call Metrics}

DeepSqueak is able to export a range of call metrics after detection of calls (Table 4.1). Some of these were used in this study, however Power, Slope, and Tonality were excluded based on their accuracy and biological relevance. Power is a metric that could provide interesting information about the strength of calls [207] however was excluded in this study because of technical variation in recording volume. Due to technical difficulties with a previous microphone that resulted in constant noise contamination (Figure 2.2), a third microphone was sourced for recording of litter 1 that resulted in lower signal detection across all recordings. This was accounted for during call detection however would directly affect call Power calculations, which are derived from amplitude decibels $(\mathrm{dB})$. Slope was excluded as calculation relies not solely on call contour, but the size and dimensions of the selection box generated around each call (Figure 2.3). Hundreds of selection boxes were redrawn and altered carefully to exclude background noise and clarify the call contour shape in each call file, so box shape was not consistent across all calls. As such, Slope as a call metric risked being heavily influenced by technical variation and was not a reliable metric. Finally, Tonality was excluded as it is also highly determinant on Power. Tonality is the representation of 'whistlelike quality' calculated by DeepSqueak to detect call contour from background noise. It requires arithmetic and geometric averaging of Power, which was already excluded based on technical inconsistency.

The calls from every eligible pup in the 6 included litters have been analysed using the indicated metrics detailed in Table 4.1. Number of Calls, Time Calling, and Call Length are all metrics that have been used in other studies to describe the effects of MIA, so were chosen to investigate. The metrics Average Principal Frequency, Change in Frequency, and Sinuosity aim to capture the complexity of each call, so were used in lieu of call classification. DeepSqueak has a call classification function however this was not used based on previous issues with poor detection ability. As mentioned earlier, machine learning programs for call detection and classification are highly reliant on the training and accuracy of the network 
used. DeepSqueak was published alongside detection networks for adult calling, however no network had been previously developed and published for pup calls. Work on a network specific to pup calls was begun by the lab of Dr Ellenbroek and is currently being retrained and improved using the detection files generated in this experiment. Before this experiment, the network had previously been developed using PND7 Sprague Dawley pup calls from a separate MIA study. The initial training of this network was not sufficiently accurate for detection of the calls in this study; paired t tests between original and manually corrected detection files for litters 2 and 3 show statistically significant differences in every call metric (all $p$ values $<0.002$, data not shown). Additionally, calls correctly identified were inaccurately shaped, with detected call contour often contaminated by background noise. As a result, hundreds of errors in each call detection file were identified and manually corrected at a high time cost. This process was necessary to ensure accurate call measurement. The time cost of additional cluster analysis for call classification was deemed too great for the scope of this thesis, particularly as the available call metrics can be used to give a broad indication of call patterns within groups. Future studies with higher power however may benefit from exploring this option.

\subsubsection{Linear Mixed Models}

As mentioned previously in this thesis, analysis of pup USVs and gene expression was carried out using linear mixed modelling (LMM). This was chosen to account for litter-related bias in the data, recommended by recent guidelines for MIA studies [125]. LMM assesses variance between groups of a random effect (in this study, variance between litters) and allows and accounts for a degree of variation between groups. This assumes that nonindependent observations within a group show similar trends to those from another (e.g. pups between litters may vary, but overall litters show similar effects) [208]. This variance is already accounted for when associating variance with main and interaction effects defined in the statistical model, essentially removing the random effect before assessing variables of interest. Accounting for litter before accounting for treatment and other relevant groups increases the likelihood that the results generated will be reproducible, as effect sizes will not be amplified by litter effects specific to this study. 
It is important to note that while effect sizes have not been amplified, they have likely been under-estimated in this study due to low sample size. While the number of pups appears relatively high ( $n=69)$, the number of litters as independent observations is low $(n=6)$. With a small independent sample size, LMMs may over-estimate the level of variance associated with litter and reduce effect sizes in main and interaction effect. Small samples are also subject to a strong shrinkage effect, where estimated means shift toward a central, global mean based on limited data for prediction [208]. Overestimation of litter effect may be particularly true in metrics with high litter variance (Figure 4.3), even more so where high variance is observed within a treatment group. This is due to the variable Litter being nested within Treatment, resulting in a practical sample size of $n=3$.

For the metrics assessed in this experiment, overall litter variance was graphed to visualise the effect this may have on USV data (Figure 4.3). Significant differences between litters of different treatment groups exist between Number of Calls, Time Calling, Average Frequency Change, and Average Sinuosity (Figures 4.3A, B, E, F). These differences are likely driven by a combination of treatment effect and natural variation. Significant differences between litters within a treatment group are of more concern, as seen in Number of Calls and Time Calling (Figure 4.3A-B). Litter-associated random variance will be highly estimated in these cases, potentially decreasing the variance associated with Treatment. Litter-associated variance may be more accurately calculated with increased litters within a treatment group. An improved estimate of litter effect would improve the prediction of all main and interaction effects, likely resulting in the nearly significant results reported in this study reaching the significance threshold (Table 4.2).

\subsubsection{Treatment Effect on Pup USVs}

Assessing treatment as a main effect on pup USVs revealed no significant effect on any of the six call metrics (Table 4.1). Treatment effect in Average Frequency Change and Sinuosity were the closest to reaching the classic significance threshold, suggesting a trend toward altered complexity of calls in poly I:C exposed pups. Additionally, a significant Treatment and 
Genotype interaction was found in Average Sinuosity, demonstrating pup genotype and treatment group only strongly affect call sinuosity when combined. When assessing pups separated into genotype and sex defined groups, one significant difference was found between treatments: Average Sinuosity of male HET calls was reduced in poly I:C exposed pups compared to saline (Figure $4.1 \mathrm{~F}, \mathrm{p}=0.038$ ). As Sinuosity is an indication of call complexity, this information all suggests male HET pups exposed to poly I:C during gestation emit less complicated USV call shapes upon separation stress.

All treatment paired groups showed a similar trend in Sinuosity, with poly I:C group call means lower than the equivalent saline group pups (Figure 4.1F). The same trend is visually represented in Number of Calls, Time Calling, and Average Frequency Change, none of which meet classic statistical significance (Figure 4.1A, B, E). Interestingly, visual differences between HET treatment groups are often larger than WTs (Figure 4.1A, C). This trend indicates a potential susceptibility might be present based on treatment and genotype, further exemplified by lower (but not significant) $p$ values reported for Treatment and Genotype interaction in these metrics (Table 4.1).

The overall treatment effect on pup calling is weaker than predicted, with most comparisons not yielding statistically significant differences. Visual trends however suggest that treatment differences may exist, potentially amplified by SERT genotype. A likely explanation for this low significance is the low number of litters used in this study, as mentioned previously. As Litter is nested within Treatment, it is possible that Treatment effect is being under-predicted due to some of the true Treatment variance being associated with Litter.

\subsubsection{Genotype and Sex Effect on Pup USVs}

Using the linear mixed effects model, Genotype was demonstrated to be the strongest main effect across all measurement types (Table 4.1). All metrics aside from Average Call Length reached statistical significance, with Sinuosity again the strongest $p$ value $(p=0.0003)$. This demonstrates PND7 pup USVs are highly influenced by SERT genotype, even with a small 
number of litters. When separated by treatment and sex, variance was most often significant between WT and HET saline group males (Figure 4.2B-E). This demonstrates a strongly significant difference in USVs between genotypes exists in males, not only in call complexity but in number of calls. The difference in males is likely driving the strength of the genotype effect, as a similar trend is observed but not significant in saline female HETs and WTs. As with Treatment this study may again be underpowered to detect a difference between female genotypes, particularly given the Sex/Genotype interactions nearing classical significance for Number of Calls and Sinuosity (Table 4.1). Interestingly, poly I:C exposed females are the only group to show a statistically significant genotype difference in Average Frequency (Figure 4.2C). Visual genotype differences are still present between the other three groups, again potentially suggesting underpowered analysis. The distribution of saline males in this figure also appears skewed due to potential outliers, likely reducing significance in this group. No outlying pups were excluded during analysis, as the low number of litters combined with large intra-and inter-litter variance makes it unclear whether pups are true outliers or simply inconsistent with the data from a low sample size.

Of all main effects, Sex was statistically the weakest (table 4.1). No Sex effect or Sex/Treatment interactions neared significance, however the relatively low $p$ values for Sex/Genotype interactions mentioned earlier suggest a difference between genotypes may be moderated by sex. When separating pups by treatment and genotype groups, saline HETs show a significant sex difference in Sinuosity only (Figure 4.2A). This difference is likely driven by the genotype effect previously observed in saline group males (Figure 4.2E), where saline HET males emit calls with far higher Average Sinuosity than all other groups visually.

\subsubsection{Final Discussion}

As mentioned throughout this discussion, treatment effect may be stronger than is reported in this model due to the litter sample size $(n=6)$. Variance within and between litters of the same treatment group is an important but rarely addressed concern for MIA models, and statistical analysis to account for this was chosen accordingly. Treatment effect in isolation was not found to be significant across any measurement however, suggesting 
genotype is the main factor affecting pup USVs in this model. Due to the small number of litters available, it is likely that the significance of treatment effects and other near-significant interactions would be improved with a higher sample size of independent litters. The results from this chapter have demonstrated that a strong SERT genotype effect exists in pup USV communication, which is potentially strongest in male pups. Furthermore, when comparing male HET pups by treatment group, a statistically significant difference between poly I:C and saline exposed pups is observed. Male HET saline pups were involved in almost all significant pairwise comparisons, with significant treatment, genotype and sex difference in Average Sinuosity. Overall, the results of this study demonstrate a clear tendency for male SERT het pups to produce more complex calls compared to others, and this is reduced in pups exposed to poly I:C during gestation. This trend further suggests that male SERT HET pups may be more susceptible to MIA-induced behavioural effects than female and SERT WT groups, however should be confirmed with further, more powerful studies. 


\section{Chapter 5: The RAC1/KAL7/DISC1 Signalosome}

\subsection{Introduction}

As discussed previously, the RAC1/KAL7/DISC1 signalosome has been implicated in SCZ and supported by animal models of NDDs (Chapter 1.4). As a short review; RAC1 is activated by the activity of guanine exchange factor KAL7, which is itself regulated by binding of DISC1 [166]. The individual components of this signalosome have all been linked to neurodevelopmental disorders, most notably DISC1 in Scottish and Finnish cohort studies $[169,171]$. Through this signalosome, it is hypothesised that altered Rac1 activity leads to changes in brain connectivity and synapse strengthening, which in turn influences phenotypic behaviours associated with ASD and SCZ. The aim of this chapter is to assess the expression of these components, and whether sex or genotype modulate any treatment effect observed. Given the wealth of evidence suggesting this pathway is dysregulated in NDDs, it is predicted that expression differences within the signalosome will be observed between treatment groups.

Gene expression is to be assessed in the following results using Quantitative Reverse Transcription Polymerase Chain Reaction (RT-qPCR). This method measures RNA abundance in a given sample (in this case, the hippocampus of PND21 pups) by converting RNA to cDNA through reverse transcription, to subsequently amplify by quantitative polymerase chain reaction (qPCR). Amplification from a sample is quantified by the thermal cycle at which amplified DNA reaches a minimum threshold fluorescence $(\mathrm{Cq})$, distinguishing it from background signal. In this study, targets of interest are averaged across technical replicates and normalised to a reference (delta $\mathrm{Cq}$ ) for comparing across groups and targets. Furthermore, this normalisation is converted to fold expression change using the $2^{\text {-deltaca }}$ method [209]. It is recommended that more than one reference gene be used, to minimise the skewing effect a poor choice of reference may produce [210]; for this reason, two reference genes were selected and geometrically averaged: Gapdh and Hprt (Table 2.4). Gapdh was selected based on its use in published literature, where primer sequences were directly borrowed from a relevant study by Owczarek et al. 2015 [167]. Hprt was also selected 
based on its current use, where it has been a successful reference gene in many studies within the Day laboratory group.

A potentially large source of variation in molecular analyses using animal tissue samples is often the quality and variation between dissections. Particularly when using brain tissue, the potential inclusion or exclusion of specific brain regions may influence expression results. This study aims to assess the hippocampus in general, as opposed to a specific region: the curved 'horn-like' structure of the rat hippocampus however could potentially lead to outer posterior and inferior sections being excluded from some samples while sampling by coronal section (Chapter 2.5) [211]. For this reason, genes expressed solely in the hippocampus were investigated to provide a quality-control measure of dissection variance. Prospero-related homeobox gene 1 (Prox1) is a protein responsible for cell fate determination of granule cells in the dentate gyrus region of the hippocampus [212]. Prox1 also functions as a mediator of adult hippocampal neurogenesis, a pathway that is potentially dysregulated in NDDs [213]. In the adult rodent brain, Prox1 is exclusively and consistently expressed in the dentate gyrus and the cerebellum [214]. As a marker of dissection variance, this was chosen to indicate the proportion of dentate gyrus present within the sample: as all samples were homogenised at the beginning of preparation (Chapter 2.8), consistently sized and regionally equal samples should contain similar expression values for this target. 


\subsection{Primer Efficiency}

Before RT-qPCR analysis, primer efficiencies were established using four-fold serial dilutions. Efficiencies were calculated for all primer sets using the following formula:

\section{Percent Efficiency $=\left(10^{\wedge}(-1 /\right.$ slope of trend line $\left.)-1\right) * 100$}

All primer sets are presented in Figure 5.1. Primer sets tested are presented with Rsquared values $\left(R^{2}\right)$ to assess dilution accuracy; every dilution series presents an $R^{2}$ value close to 1 , demonstrating the standard curve used to calculate primer efficiencies are accurate. All primer sets in this study were found to have a primer efficiency between $80-100 \%$. General guidelines for RT-qPCR primers indicate that primer efficiencies should fall between $80-120 \%$, with $100 \%$ indicating a full doubling of target transcript with every PCR cycle [215]. Additionally, MIQE guidelines for RT-qPCR indicate all primer sets should have a similar efficiency to the reference gene primers (Gapdh and Hprt) to allow for confident comparisons between targets [210]. As these efficiencies fall within a small and generally accepted range, they were deemed acceptable for future use.

Melt peaks for each primer set were used to confirm primer specificity (Figure 5.2). All primer sets present one sharp peak per reaction, consistent between samples (data not shown) and technical duplicates. All reactions show no small peak preceding the main peak, which would indicate primer dimerization. Melt temperatures for each product are similar, which is expected given the similar amplicon size targeted by each primer set. The consistency and steep curves produced by each reaction suggest each primer is specific to one target, with no mis-priming of a second unintended target. 


\section{Figure 5.1: Primer Efficiencies for RT-qPCR Primer Sets}
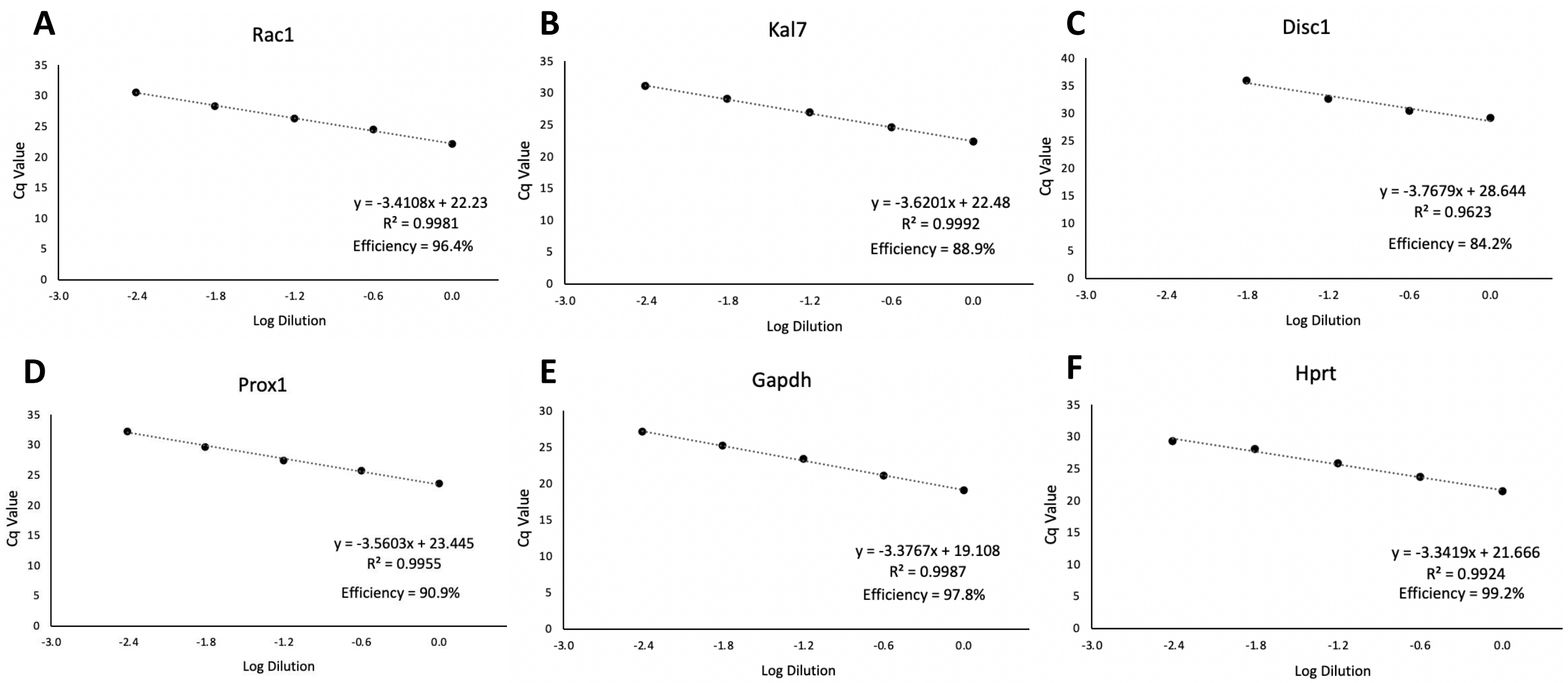

Figure 5.1: Primer Efficiency tests are presented for each gene assessed by RT-qPCR. 5 4-fold dilutions were analysed and plotted as log dilution against amplification Cq values. Disc1 (C) did not amplify within the set 40 cycles at the lowest concentration dilution, so was calculated from 4 dilution points. All figures are presented with trend line equations, $R^{2}$ values, and calculated primer efficiencies. 
Figure 5.2: Melt Curve Analyses for RT-qPCR Primer Set Products
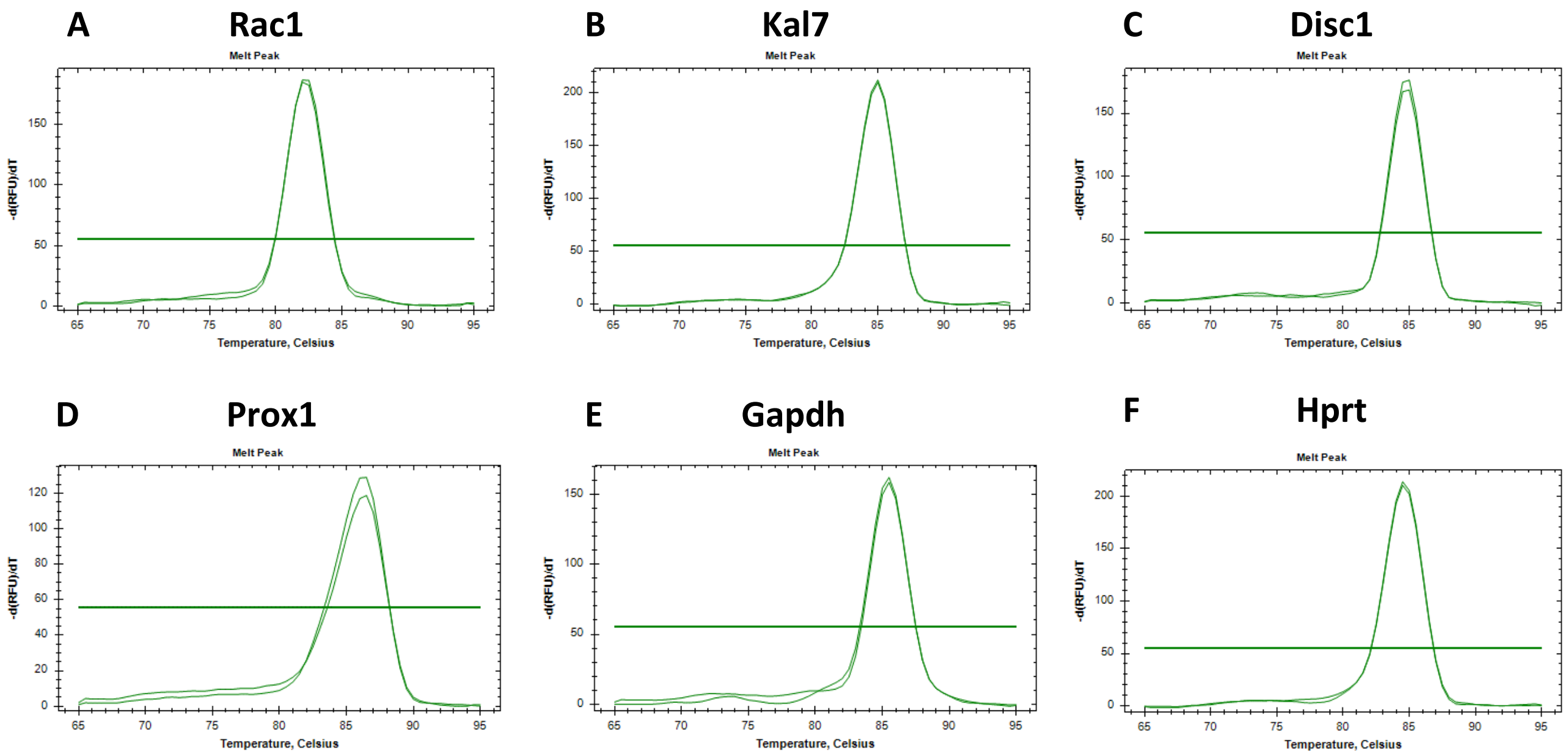

Figure 5.2: Melt Curves for each primer set are presented, taken from duplicate RT-qPCR reactions. All primer sets show a single melt peak, implying a single PCR product per reaction. 


\subsection{Gene Expression}

\section{Table 5.1: Overall Effects on Gene Expression Targets}

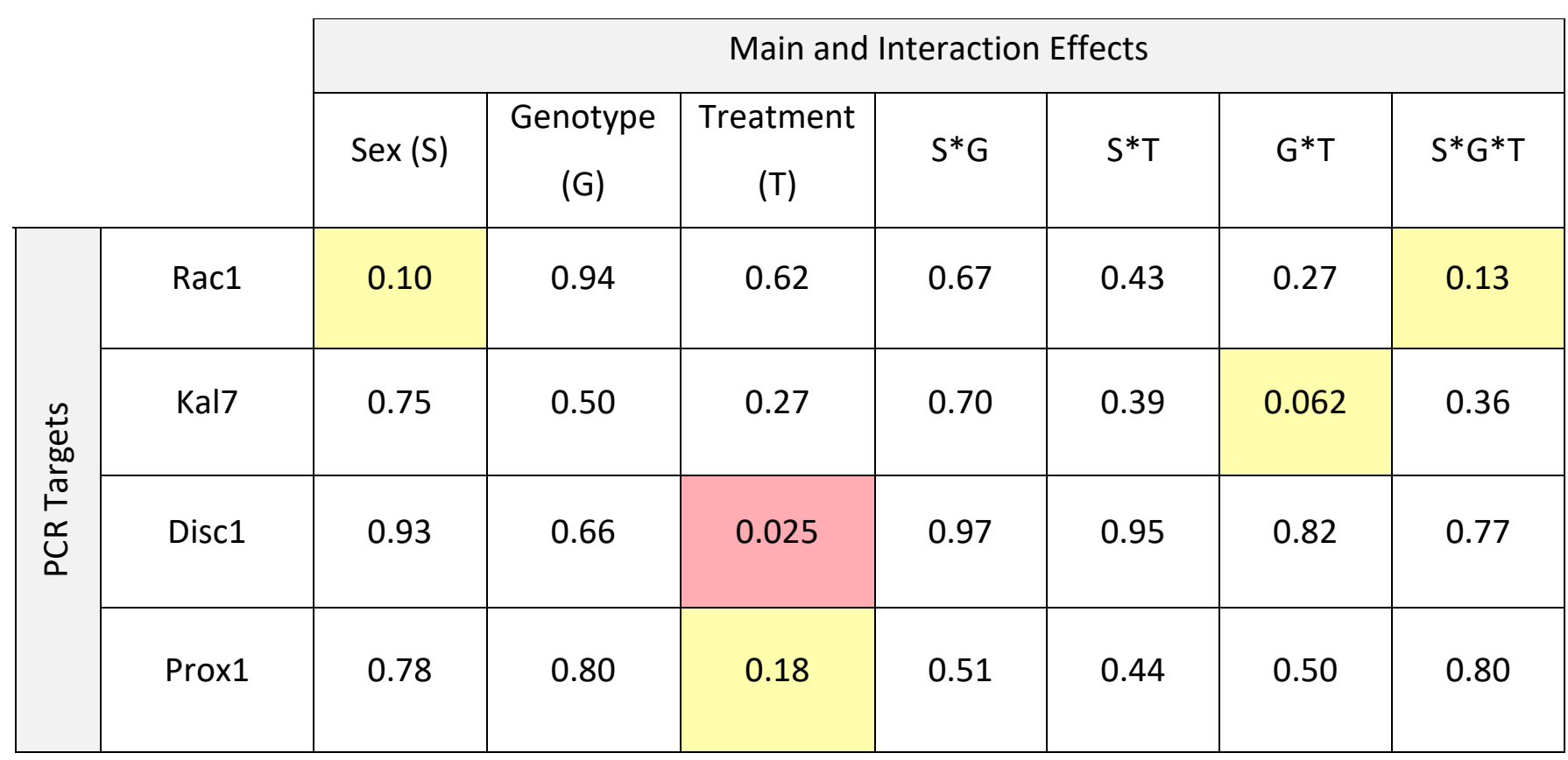

Table 5.1: All $p$ values resulting from one-way ANOVAs in each linear mixed model are presented for all targets in this experiment (pup $n=22$ ). Significant results are highlighted in red $(p<0.05)$, while those near significance are presented in yellow $(p<0.20)$. All values are presented to 2 significant figures, up to 4 decimal places. All models result in numerator degrees of freedom of 1 , with denominator degrees of freedom differing in effect (Treatment effect $d f=3$, All other effects $d f=11$ ). F-statistics for each model are presented in Appendix 2.

Linear mixed modelling of the results in this study reveals a significant treatment effect in Disc1 expression ( $p=0.025)$, with no other significant effects reported across all models (Table 5.1). Disc1 expression does not near significance in any other main effects or interactions. A sex-specific effect approaches statistical significance in Rac1 expression $(p=0.10)$, with no other genes appearing to be affected by Sex. Rac1 additionally nears significance for a three-way interaction of Sex, Genotype, and Treatment $(p=0.13)$, suggesting an effect when assessing pups in specific sex/genotype/treatment groups. Kal7 expression does not show significance across all effects tested, however nearly reaches significance in 
the genotype and treatment interaction $(p=0.062)$. Interestingly, Prox 1 expression approaches but does not meet significance in treatment effect $(p=0.18)$, suggesting the possibility of Prox1 expression differing between treatment groups.

Importantly, Prox1 and Kal7 models pass normality and equal variance tests (as defined in 2.10) while the Rac1 and Disc1 models fail Levene's test for homogeneity of variances ( $p<$ $0.001, f=12.84$ and $p=0.002, f=5.95$ respectively). Under further assessment of Sex, Genotype, and Treatment groups, residuals in Rac1 and Disc1 each vary significantly by only one group. Rac1 varies significantly by Genotype $(p=0.005, f=16.8)$, but does not reach significance in Sex or Treatment $(p=0.19, f=1.78$ and $p=0.08, f=3.41$ respectively). Disc1 alternatively only shows significant variation in $\operatorname{Sex}(p=0.009, f=14.90)$ while not in Genotype or Treatment $(p=0.39, f=0.56$ and $p=0.36, f=0.38$ respectively). This may cause some misrepresentation of effect sizes, particularly in relation to the factor which shows unequal variance (Genotype effects in Rac1 expression and Sex effects in Disc1 expression). This violation requires some caution in interpreting model results, however does not discount the model entirely; linear mixed models tend to be resilient to violations of variance and normality assumptions [216]. 


\section{Prox1 Expression By Researcher}

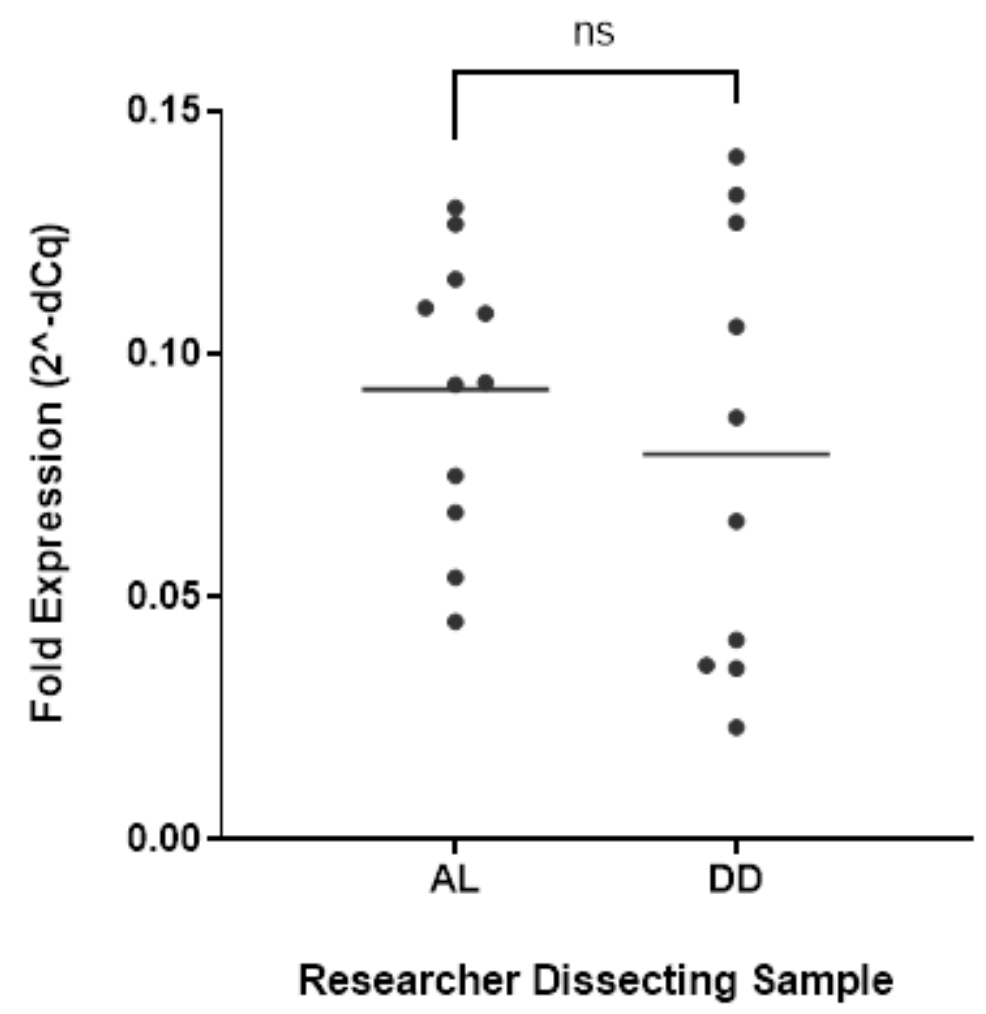

Figure 5.3: Expression of Prox1 is grouped by the individual researcher who carried out dissection for each animal ( $A L=$ Alex Lister, $D D=D r$ Darren Day). No significant difference is found between researcher by students $t$ test $(p=0.428)$.

As Prox1 was selected to indicate dissection consistency, Prox1 was additionally compared between researchers who carried out dissections (Figure 5.3). Pups from each treatment, sex, and genotype group were dissected by both Dr Day and myself. No statistically significant difference in Prox1 expression is presented between researchers when calculated by students t test $(p=0.428)$. Both dissection groups also show a similar level of variance in expression. This suggests that inconsistency in dissection does not play a significant role in the findings presented in this chapter. 


\section{Hippocampal Rac1/Kal7/Prox1 Expression}

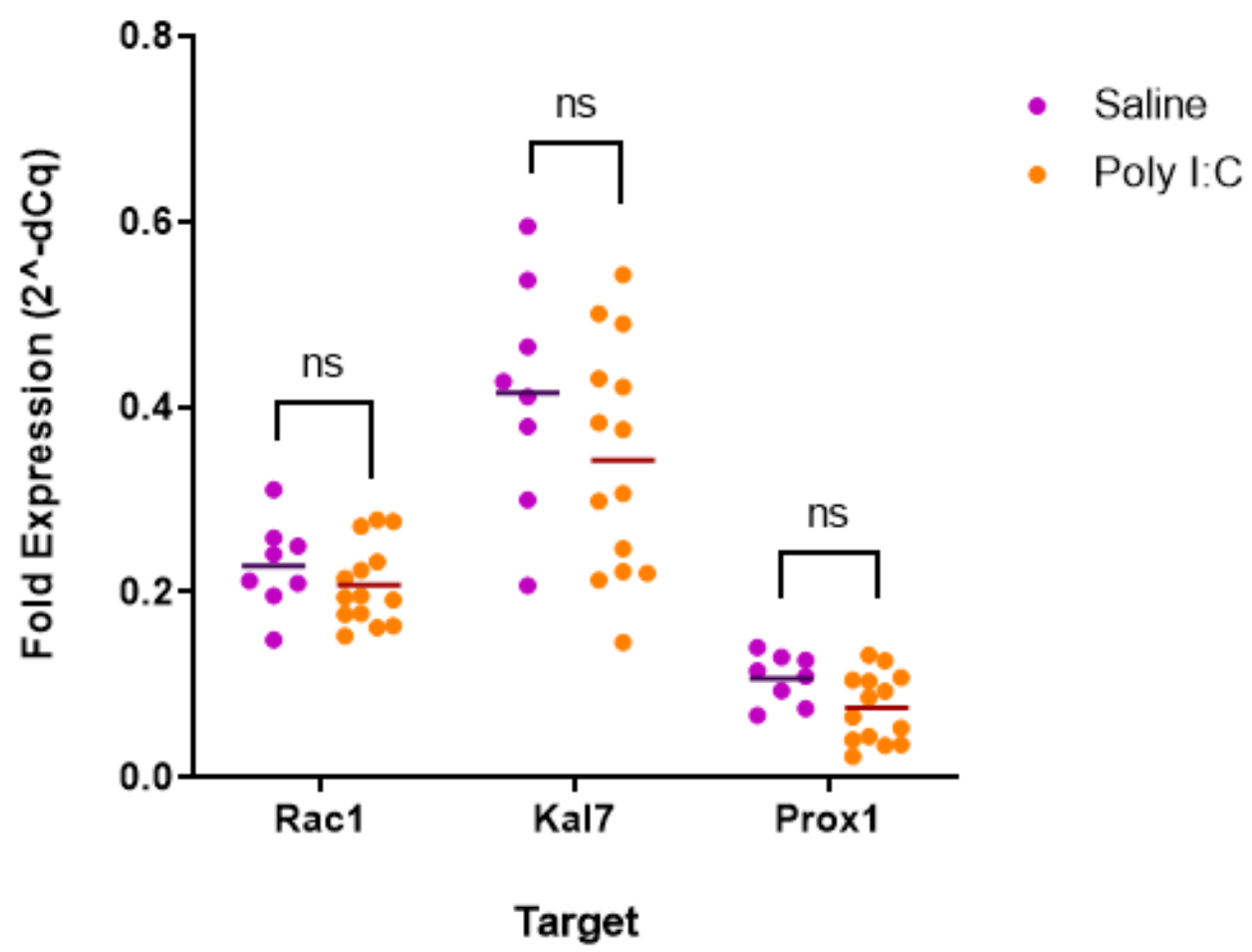

Figure 5.4: Fold expression of Rac1, Kal7, and Prox1 in PND21 pups is compared by treatment group. No differences between poly I:C and saline group pups met statistical significance when calculated using linear mixed modelling with Treatment as the only main effect (Rac1: $p=0.58, f=0.39$; Kal7: $p=0.30, f=1.59 ;$ Prox1: $p=0.13, f=4.15$ ).

To investigate the effect of treatment in gene expression of Rac1, Kal7, Prox1, and Disc1, linear mixed models were simplified to consider only treatment as a main effect (excluding sex, genotype, and interactions). This was carried out to maximise accuracy of the treatment estimate, as statistical conclusions only considering one effect may become misleading when drawn from a model accounting variation to multiple effects. Across all genes assessed, only Disc1 shows a significant difference between treatment group $(p=0.016)$. Visual assessment of Rac1, Kal7, and Prox1 shows a trend toward decreased expression, however this is not 
confirmed statistically. Additional modelling grouping pups by Sex and Genotype in isolation (as done with treatment) reveals no statistically significant comparisons. Pups were not statistically assessed by Sex, Genotype, and Treatment clustering as seen in Chapter 4 due to a low sample size of pups per group. Pups differentiated by sex and genotype within each treatment group alongside visual observations can be found presented as Figure A4.1 in Appendix 4.

\section{Figure 5.5: Disc1 Expression}

\section{Hippocampal Disc1 Expression}

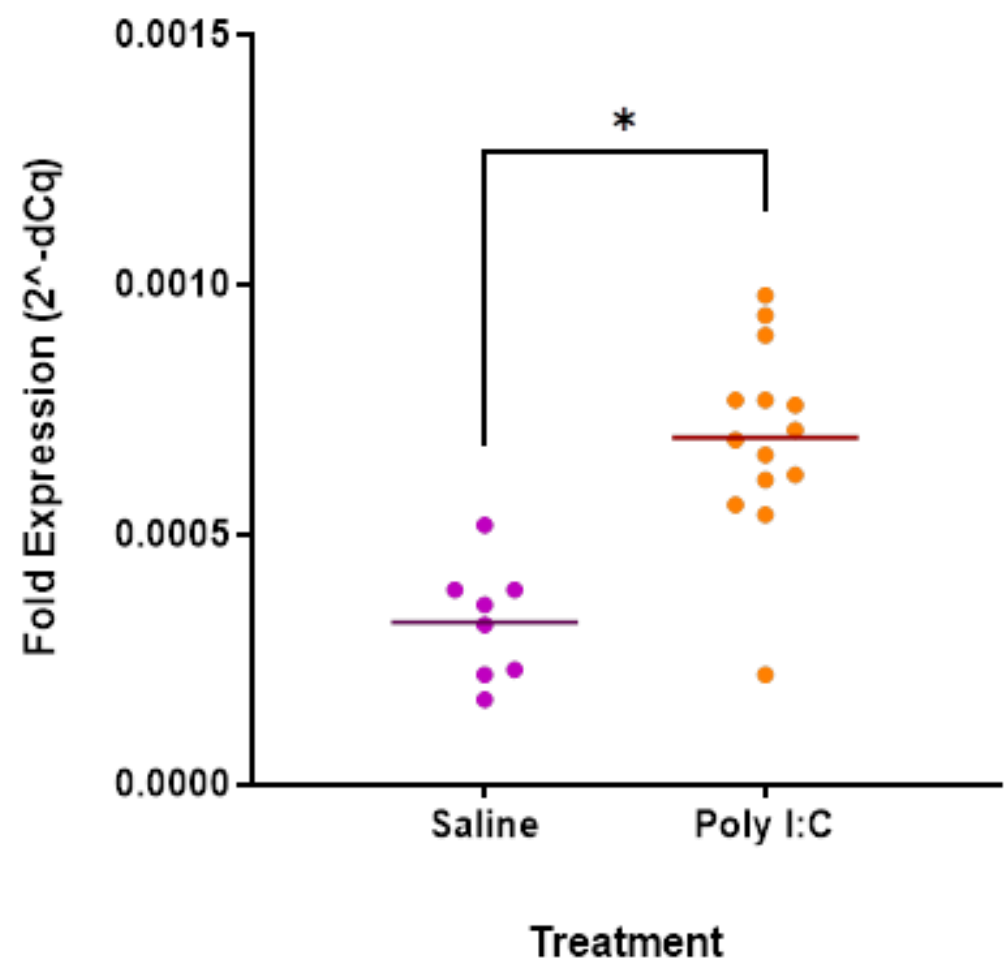

Figure 5.5: Fold expression of Disc1 in PND21 pups is compared by treatment group. Using linear mixed modelling with Treatment as the main effect, a statistically significant increase in Disc1 expression is observed in poly I:C exposed pups $(p=0.016, f=24.37)$. 


\subsection{Discussion}

\subsubsection{Validity Measures}

For this experiment, three relevant measures of validity were assessed. The first was primer efficiencies, to validate the results generated by qRT-PCR. All primer efficiencies under the parameters specified in Chapter 2.8 were between $80-100 \%$, with reference genes near $100 \%$ efficiency. This is considered within an acceptable range for RT-qPCR studies, as efficiencies are high and similar enough to result in results being closely representative of the original cDNA concentration present within each sample. Additionally, all primer sets show one melt peak per reaction, consistent across all RT-qPCRs performed in this study (Figure 5.2). This suggests only one product per reaction was amplified, and primers show specificity to one target. These were further confirmed to only have one product at expected size by gel electrophoresis (data not shown).

Following primer efficiencies, the Prox1 primer set was designed to be a measure of dissection consistency. As mentioned in section 5.1, Prox1 is a gene expressed in the adult rat brain exclusively in granule cells specific to the dentate gyrus region of the hippocampus, as well as in the cerebellum [214]. As the dentate gyrus is located across both in the posterior and ventral portions of the hippocampus, it was likely the region most subject to dissection variance across animals and researchers carrying out dissections when taking coronal sections (Section 2.5.2, Figure 2.4). Hippocampus samples used in this analysis were dissected by one of two researchers (Dr Day, $n=10 ;$ Alex Lister, $n=12$ ) across all treatment, genotype, and sex groups.

To assess potential variation, Prox1 expression was measured in every sample. No significant differences were observed in Prox1 across main and interaction treatment effects, although neared classic statistical significance in Treatment effect (Table 5.1). When simplifying the model and only comparing pups by treatment, differences again were not significant (Figure 5.4). Due to the low sample size across pups and litters it cannot be determined whether this effect is truly non-significant, or this study is too underpowered to 
detect significance. There are no differences reported between sex and genotype groups, and additionally no visual skewing between dissectors (Figure 5.3). Finally, variance in Prox1 expression is not over a relatively large range. This suggests any variance in Prox1 expression that may exist between treatments is not due to high dissection inconsistency, either between individual animals or between researchers. As Prox1 is associated with adult neurogenesis in the hippocampus, which may be involved in altered connectivity and plasticity in NDDs [213], it is conceivable that Prox1 expression is altered following MIA. Even in this situation, Prox1 can still be used to assess variation of dissection samples within treatment groups.

Finally, validation tests during statistical analysis were carried out to confirm the assumptions of linear mixed modelling. During analysis of USVs, all modelled metrics met the assumptions of normality (a non-significant result in Shapiro's test) and equal variances (a non-significant result in Levene's test). During analysis of RT-qPCR results, only Prox1 and Kal7 models passed both tests. Rac1 and Disc1 models by contrast failed to meet the assumption of equal variances between groups, producing statistically significant results through Levene's test for homogeneity of variances. This is of concern as heteroscedasticity (unequal variance) may lead to inaccurate calculation of predictive error, however linear mixed models are robust to homoscedasticity assumption violations [216, 217]. The model weakens in predictive power when the unequal variance is associated with an main effect being modelled, so results in these effects should be reported with caution [217]. Specifically in this data, this relates to Genotype effects in Rac1 expression and Sex effects in Disc1. It should also be noted, just as discussed in USV analysis, that the overall sample size of independent observations is low ( $n=6$ litters). This leads to the results of this study likely being underpowered, potentially not strong enough to detect changes of a smaller effect size.

\subsubsection{Signalosome Expression}

A total of 22 pups were used in this analysis, however the categorical grouping of pups by sex, genotype, and treatment result in a sample size per group of 2-4 pups. This is not sufficient to make valid statistical claims between the 8 groups, so results were not assessed 
for complex pairwise interactions as carried out during USV analysis. Instead, analysis of gene expression across the 4 targets were focused mainly on the effect of treatment. Pups were also visually assessed within treatment group, to briefly investigate the dispersal of sex and genotype groups within treatment. When viewing the distribution of pups within the poly I:C group, no strong sex or genotype skewing is visible. In saline controls, a strong skewing of data points is visible by genotype in Kal7 expression, and slight skewing by sex is visible in Rac1 expression (Figure A4.1). This was not assessed statistically given the low sample size but is an trend that may provide interesting results in a larger sample size.

Of all three components of the Rac1/Kal7/Disc1 signalosome, Disc1 was the only target to show a statistically significant shift in expression due to treatment (Figure 5.5). Disc1 expression was shown to be significantly increased in poly $\mathrm{I}: \mathrm{C}$ exposed pups compared to saline controls $(p=0.016)$, with mean expression approximately doubling. It should be noted that fold expression is small, expressed on a much lower scale than the other genes investigated. This difference however is extremely robust, with only one data point overlapping between groups. Visually, slight decreases are observed but not statistically supported in Rac1 and Kal7 expression in poly I:C exposed pups (Figure 5.4). This lack of significance is potentially due to the small sample size of litters, as was predicted in Chapter 4. No significant effects are observed when comparing overall sex or genotype differences.

A significant increase in Disc1 expression suggests increased inhibition of the Rac1/Kal7/Disc1 signalling pathway. Using the assumption that increased expression results in an equivalent increase in translation and protein abundance, this would result in a hippocampal increase in Disc1. Increased Disc1 abundance would likely result in increased sequestering of Kal7 to Psd95, preventing activation of Rac1. Importantly, one Kal7 protein may act as a guanine exchange factor (GEF) for multiple Rac1 proteins within the cell; a small increase in Disc1 inhibition of Kal7 may therefore have a large effect in reducing the activity in this pathway. Down-regulation of this pathway would also be supported by a decrease in Rac1 and Kal7 expression; this is not statistically supported by this data, but a visual trend is observed. It is important to note that altered Disc1 expression is an indication of a regulatory change, however does not provide evidence that activation of Rac1 is altered. Further studies 
to ensure protein abundance is also increased would provide an important validatory step to confirm these RT-qPCR findings. 


\section{Chapter 6: General Discussion}

\subsection{Overall Conclusions}

\subsubsection{Model Validation and Statistical Analysis}

Primary tests carried out in this model were chosen to validate the presence and effects of maternal immune activation as they are commonly reported in published literature. Temperature was the primary validation tool used, to identify whether mothers treated with poly I:C experienced a temperature increase/fever. Shortly after exposure poly I:C treated dams present a statistically significant increase in temperature while saline exposed dams do not, supporting the presence of MIA in these animals (Figure 3.1). Maternal weight was also assessed, however no maternal weight loss was observed as commonly reported (Figure 3.2). As an initial measure of altered pup development, weight gain in pups between PND7 and PND21 was also assessed. While an overall treatment effect was initially observed (Figure 3.4), the strongest observed effect on pup weight was litter size at birth (Figure 3.5). Pups across treatments and litters did not differ in the percentage of weight gained based on their size at PND7, suggesting physical growth was not delayed by poly I:C exposure during gestation.

This final analysis of pup weight drew particular attention to the role of litter-based variation in rodent research models, and the importance of disclosing more immune activation and litter demographic information than is currently expected in published research. High variation exists in MIA studies in terms of quantifying and validating immune response, with numerous reviews of current literature supporting the need for more robust and comparative testing across studies [125]. The need for clearer information is also true of experiments using MIA-exposed pups. Often, factors such as litter number, size, and sex composition are not clearly specified or discussed in published research. This makes it difficult to determine whether any litter-based variation is skewing published data, especially given that litter size had a significant effect on pup growth in this study, amplifying treatment effect. 
Based on the above findings, behavioural and molecular analyses were carried out using linear mixed modelling to account for litter variation as a 'random effect'. Commonly in published research, pup experiments are assessed by ANOVA or other tests that assume independence between observations. These analyses therefore define independent sample size as the number of pups analysed, however this is arguably incorrect [189]. Within rodent research, multiparous species are often considered highly similar within a litter and may be considered non-independent. This argument is strengthened in MIA models, where treatment is applied to mothers and thus a whole litter at once [177]. An alternative and recommended method for analysis is to consider litter number as sample size and assess pups similarly to technical replicates. This is not possible if subgroups within a litter are of biological interest, such as sex or genotype (or both, as is the case in this thesis). Instead, the strongest statistical measure of the variation in this model was mixed linear modelling; using this method, the independence assumption can be met through marking clusters of nonindependent data as 'random effect' groups. While application of this modelling had mixed success in this study (see 6.2.1), the statistical methods employed were the most appropriate given the structure of this study and the animals used.

It is important to note that linear mixed models are often simplified by removing terms with little effect, chosen based on best predictive fit using methods such as the Akaike Information Criterion (AIC) [218]. This was not carried out in this study as all main and interaction effects in the full model are of biological interest, and relevant to the design of the experiment. The central aim of this study is to assess treatment effect in this model and whether this is modified by genotype and potentially sex; these main effects, and by extension interactions between them, are all important factors to be considered. While simpler models may more clearly have accounted for variation (for example excluding sex from models where no sex effects are detected), the aims of this thesis require assessment of all the variables present in the full model. 


\subsubsection{Behavioural and Molecular Experiments}

Results from the behavioural study in this thesis suggest that strong genotype differences exist between SERT WT and HET pups, particularly in male offspring (Table 4.2). This is true across multiple metrics, both related to rate of calling and call complexity. SERT HET pups exposed to poly I:C lose this contrast between genotypes, with significant differences observed between male HETs across treatment group (Figure 4.1). The significant interaction observed in Average Sinuosity between genotype and treatment further supports the hypothesis that treatment and genotype both contribute to behavioural change. Importantly, genotype had the strongest effect associated with altered calling behaviour, while treatment neared but did not reach statistical significance in metrics associated with call complexity. As discussed throughout this thesis, this is likely attributable to low statistical power to detect treatment effect. The strong altered behaviour pattern observed between genotypes, particularly in male HET pups, provides further evidence to support the body of literature indicating reduced SERT expression may lead to increased stress/anxiety responses [144-146].

Molecular analysis of expression of the Rac1/Kal7/Disc1 pathway in this model revealed a small but robust significant treatment difference in Disc1 expression (Figure 5.5), with no other significant treatment effects observed (Table 5.1). The finding that Disc1 expression is significantly affected by treatment aligns with current evidence in SCZ models, which suggest altered Disc1 expression and subsequently altered activity of the Rac1/Kal7/Disc1 signalosome. No significant expression effects were observed across Sex and Genotype, although Rac1 expression approached significance in Sex effect and in the Sex/Genotype/Treatment interaction. No statistically significant evidence is presented to suggest genotype effect or interaction in expression of this signalosome. Results from this study therefore do not support the hypothesis that genotype modulates the effect of treatment on this signalosome, however do support the hypothesis that treatment may cause expression changes in this signalosome. As this analysis is underpowered both by litter and pup sample, conclusions from this study should be considered with caution; these findings do 
however provide initial evidence that suggest immune activation during pregnancy may affect this signalling pathway in offspring.

\subsubsection{Thesis Aims}

The overall aim of this research was to assess whether SERT genotype would modulate the effects of maternal immune activation (MIA) during gestation. This was to model human variation in SERT expression associated with the polymorphic region of the 5-HTTLPR, where the VNTR-S allele causes reduced SERT expression. As neurodevelopmental disorders such as ASD and SCZ are both associated with MIA and altered serotonin signalling, it was hypothesised that an animal model subject to both MIA and reduced SERT may show stronger behavioural and molecular alterations than MIA alone. Overall, the findings of this model suggest that behavioural effects may be strongest in offspring both exposed to MIA and reduced SERT, particularly in male subjects. Molecular analysis identifies increased Disc1 expression in MIA-exposed offspring, suggesting a treatment effect leading to reduced Rac1 activation through this pathway. Due to a low number of litters, it is likely that this study is underpowered to fully detect treatment and genotype effects across experiments; this is particularly true of molecular analysis where the high number of categorical groups (defined by sex, genotype, and treatment) demand a higher sample size of pups than was available within the time frame of this thesis. The findings of this study that do meet classic statistical significance suggest SERT genotype may play a role in modulating the behavioural effects of poly I:C induced MIA, however cannot conclude whether this is also true of the molecular changes in the Rac1/Kal7/Disc1 signalosome. Importantly, this research provides initial evidence to suggest increased Disc1 expression in models of MIA, and a link between MIAassociated behavioural changes and altered SERT expression. Furthermore, this research suggests a connection between the serotonergic system and MIA susceptibility may exist in animal models of neurodevelopmental disorders and warrants further investigation. 


\subsection{Study Limitations}

\subsubsection{Sample Size and Statistical Power}

While a high level of confidence exists in the statistical methods chosen, it should be noted that the low number of litters $(n=6)$ included in this study limit the ability of the model in making confident assumptions. Results from many of the behavioural and molecular analyses approached but did not reach classic statistical significance $(p=0.05)$. This is likely due to the low number of litters causing the study to be underpowered, resulting in effects being under-estimated. A low number of litters was originally chosen with the intention of using a maximum number of animals from each litter, minimising the number of breeding pairs needed to align with ethics guidelines. Given the nature of the data produced, this weakened the overall power of the experiment as large portions of the data produced were deemed non-independent. Instead, using more litters may improve the power of the model to produce more conclusive and confident results. This could be balanced with ethical guidelines by still using a high number of pups from each litter. To manage time constraints (particularly in studies similar to USV analysis where time and labour cost is high) pups of a litter could potentially be split across experiments; this would also minimise pup handling through development, which has been historically associated with altered behaviour [219].

\subsubsection{Maternal Immune Response}

While maternal immune response was confirmed through temperature change in dams following injection, variation was seen in the height of this temperature change. Biological variation in immune response is a likely factor in this, as each dam has a unique immune system and will respond to poly I:C exposure with fevers of different length and height. This was a primary concern when assessing pups in later experiments, leading to the selection of a statistical method to account for litter variance. Strength of MIA as a response to poly $\mathrm{I}: \mathrm{C}$ is considered one of the largest contributors to contradictory evidence being presented in current literature [178]. Validating immune response has occurred is an important step in placing this model within current research, however more validation work could be done in 
future explorations of this model. Specifically, carrying out dose-response tests and analysis of poly I:C reagent prior to use would provide useful information as to the relative strength of immune response. This could also be investigated further by direct cytokine analysis of mothers; this may also contribute to maternal stress through invasive sampling however and confound the effect of treatment. Due to time constraints, these tests were considered outside the scope of this thesis however should be considered for future projects.

\subsubsection{Animal Models in Human Neurological Disorders}

It should be noted that animal models have a limited ability to model human disorders, both in behavioural alteration and molecular mechanisms. Model animals such as Rattus norvegicus can provide a simplified and more ethical model than assessing changes in humans; in particular, the ability to control and manipulate variables such as maternal immune activation cannot be carried out in human subjects. Additionally, animal studies allow assessment of environmental and genetic factors in living systems and across developmental stages that are unable to be fully explored by other techniques. While animal models have many benefits, they also face many challenges in translatability to human disorders. This is partially attributed to common misuse and misrepresentation of statistical models in published research leading to irreproducible results, and also by the limited behavioural and biological similarity between humans and rodent models [220].

While influences such as MIA may be observed in both animals and humans, difficulty lies in translating animal models to human conditions. Disorders such as ASD and SCZ already display complicated and variable phenotypes, with diagnosable behaviours ranging across individuals and the potential for episodic symptom changes. These nuances cannot be accurately modelled in animals; instead, animal research relies on detecting modified communicative and physical behaviour in the ASD of SCZ model group in comparison to controls [221]. These are often in broad categories to relate to general symptoms of human disorders, such as the use of pup USVs to indicate altered social communication during early life. Additionally, molecular mechanisms within the brain differ between humans and animal models. While there is a level of broad similarity between the two, human circuits have 
additional complexity which is unable to be assessed in Rattus norvegicus. For example, in humans the long non-coding RNA DISC2 has been associated with regulation of DISC1 expression. This is highly relevant to the RAC1/KAL7/DISC1 signalosome, however no DISC2 equivalent has been identified in rodents [168]. Many ASD and SCZ related genes are similar in this regard, for example the synapse stabilising protein NLGN4 does not have an identified equivalent in Rattus norvegicus [222]. While the findings of this model suggest a behavioural and molecular changes as a result of MIA exposure during gestation, this must be interpreted understanding the animal model cannot directly translate to the complexity of human biology. 


\subsection{Future Directions}

\subsubsection{Continued Analysis of the Rac1/Kal7/Disc1 Pathway}

The results from this study have revealed a strong genotype effect in the USVs of PND7 pups, with a significantly higher number of calls and more complex calls produced by HETs compared to WTs. This effect is significantly presented in males, with a similar trend mirrored in females. This study has also demonstrated an increased susceptibility for altered social communication in male SERT HET pups exposed to MIA during gestation. The overall trend, while only reaching statistical significance in Average Sinuosity, suggests that HET pups exposed to MIA produce less-complex calls in response to separation stress. Further study in this area is recommended; adding to the number of litters analysed will improve the predictive power of the linear mixed models used in this analysis and increase confidence in these results.

Additionally, this model has presented a significant treatment difference in Disc1 expression, with a small but robust increase in Disc1 present in pups exposed to MIA. This area warrants further investigation, to fully investigate the effects of sex and genotype in the MIA model. This study is intended to continue within this laboratory, by expanding this RTqPCR study to include a larger sample size and a secondary time point (PND60, to assess whether changes observed at PND21 persist into adulthood). Furthermore, RT-qPCR is an indication of gene expression, and does not directly assess activity in this pathway. Quantification of Rac1 activation in this model would complement RT-qPCR analysis, providing a more comprehensive image of any potential dysregulation following MIA. This may also be paired with protein quantification of signalosome components, to confirm changes in gene expression correlate to changes in protein abundance. 


\section{References}

1. Morris-Rosendahl, D., Neurodevelopmental disorders - the history and future of a diagnostic concept. Dialogues in Clinical Neuroscience, 2020. 22(1): p. 65-72.

2. Birnbaum, R. and D.R. Weinberger, Genetic insights into the neurodevelopmental origins of schizophrenia. Nature Reviews Neuroscience, 2017. 18(12): p. 727-740.

3. Association, A.P., Diagnostic and statistical manual of mental disorders (DSM- $\left.{ }^{\circledR}\right)$. 2013: American Psychiatric Pub.

4. Grzadzinski, R., M. Huerta, and C. Lord, DSM-5 and autism spectrum disorders (ASDs): an opportunity for identifying ASD subtypes. Molecular Autism, 2013. 4(1): p. 12.

5. Rapoport, J., et al., Autism Spectrum Disorders and Childhood-Onset Schizophrenia: Clinical and Biological Contributions to a Relation Revisited. Journal of the American Academy of Child \& Adolescent Psychiatry, 2009. 48(1): p. 10-18.

6. Green, M.F., W.P. Horan, and J. Lee, Social cognition in schizophrenia. Nature Reviews Neuroscience, 2015. 16(10): p. 620-631.

7. Bowie, C.R. and P.D. Harvey, Cognitive deficits and functional outcome in schizophrenia. Neuropsychiatric Disease and Treatment, 2006. 2(4): p. 531-536.

8. Owen, P.R., Portrayals of Schizophrenia by Entertainment Media: A Content Analysis of Contemporary Movies. Psychiatric Services, 2012. 63(7): p. 655-659.

9. Patel, V. and S. Saxena, Transforming Lives, Enhancing Communities - Innovations in Global Mental Health. New England Journal of Medicine, 2014. 370(6): p. 498-501.

10. Owen, M.J., A. Sawa, and P.B. Mortensen, Schizophrenia. The Lancet, 2016. 388(10039): p. 86-97.

11. Trevisan, D.A., et al., Autism Spectrum Disorder and Schizophrenia Are Better Differentiated by Positive Symptoms Than Negative Symptoms. Frontiers in Psychiatry, 2020. 11.

12. Chiarotti, F. and A. Venerosi, Epidemiology of Autism Spectrum Disorders: A Review of Worldwide Prevalence Estimates Since 2014. Brain Sciences, 2020. 10(5): p. 274.

13. Charlson, F.J., et al., Global Epidemiology and Burden of Schizophrenia: Findings From the Global Burden of Disease Study 2016. Schizophrenia Bulletin, 2018. 44(6): p. 1195-1203.

14. Loomes, R., L. Hull, and W.P.L. Mandy, What Is the Male-to-Female Ratio in Autism Spectrum Disorder? A Systematic Review and Meta-Analysis. Journal of the American Academy of Child \& Adolescent Psychiatry, 2017. 56(6): p. 466-474.

15. Abel, K.M., R. Drake, and J.M. Goldstein, Sex differences in schizophrenia. International Review of Psychiatry, 2010. 22(5): p. 417-428.

16. van der Werf, M., et al., Systematic review and collaborative recalculation of 133693 incident cases of schizophrenia. Psychological Medicine, 2014. 44(1): p. 9-16.

17. Mendrek, A. and A. Mancini-Marie, Sex/gender differences in the brain and cognition in schizophrenia. Neuroscience \& Biobehavioral Reviews, 2016. 67: p. 57-78.

18. Lai, M.-C. and P. Szatmari, Sex and gender impacts on the behavioural presentation and recognition of autism. Current Opinion in Psychiatry, 2020. 33(2).

19. Jamain, S., et al., Mutations of the $X$-linked genes encoding neuroligins NLGN3 and NLGN4 are associated with autism. Nature Genetics, 2003. 34(1): p. 27-29. 
20. Niu, M., et al., Autism Symptoms in Fragile X Syndrome. Journal of Child Neurology, 2017. 32(10): p. 903-909.

21. Jacquemont, S., et al., A Higher Mutational Burden in Females Supports a "Female Protective Model" in Neurodevelopmental Disorders. The American Journal of Human Genetics, 2014. 94(3): p. 415-425.

22. Hernandez, L.M., et al., Imaging-genetics of sex differences in ASD: distinct effects of OXTR variants on brain connectivity. Translational Psychiatry, 2020. 10(1).

23. Sokolova, E., et al., A Causal and Mediation Analysis of the Comorbidity Between Attention Deficit Hyperactivity Disorder (ADHD) and Autism Spectrum Disorder (ASD). Journal of Autism and Developmental Disorders, 2017. 47(6): p. 1595-1604.

24. Zheng, Z., P. Zheng, and X. Zou, Association between schizophrenia and autism spectrum disorder: A systematic review and meta-analysis. Autism Research, 2018. 11(8): p. 1110-1119.

25. Upthegrove, R., S. Marwaha, and M. Birchwood, Depression and Schizophrenia: Cause, Consequence or Trans-diagnostic Issue? Schizophrenia Bulletin, 2016: p. sbw097.

26. Etchecopar-Etchart, D., et al., Comorbid Major Depressive Disorder in Schizophrenia: A Systematic Review and Meta-Analysis. Schizophrenia Bulletin, 2021. 47(2): p. 298308.

27. Temmingh, H. and D.J. Stein, Anxiety in Patients with Schizophrenia: Epidemiology and Management. CNS Drugs, 2015. 29(10): p. 819-832.

28. Mingins, J.E., et al., Anxiety and intellectual functioning in autistic children: $A$ systematic review and meta-analysis. Autism, 2021. 25(1): p. 18-32.

29. Rai, D., et al., Association Between Autism Spectrum Disorders With or Without Intellectual Disability and Depression in Young Adulthood. JAMA Network Open, 2018. 1(4): p. e181465.

30. Chaste, P. and M. Leboyer, Autism risk factors: genes, environment, and geneenvironment interactions. Dialogues in clinical neuroscience, 2012. 14(3): p. 281-292.

31. Estes, M.L. and A.K. McAllister, Maternal immune activation: Implications for neuropsychiatric disorders. Science, 2016. 353(6301): p. 772-777.

32. Ronald, A. and R. Hoekstra, Progress in Understanding the Causes of Autism Spectrum Disorders and Autistic Traits: Twin Studies from 1977 to the Present Day. 2014, Springer New York. p. 33-65.

33. Hallmayer, J., Genetic Heritability and Shared Environmental Factors Among Twin Pairs With Autism. Archives of General Psychiatry, 2011. 68(11): p. 1095.

34. Anderson, G.M., Twin Studies in Autism: What Might They Say About Genetic and Environmental Influences. Journal of Autism and Developmental Disorders, 2012. 42(7): p. 1526-1527.

35. Hilker, R., et al., Heritability of Schizophrenia and Schizophrenia Spectrum Based on the Nationwide Danish Twin Register. Biological Psychiatry, 2018. 83(6): p. 492-498.

36. Farrell, M.S., et al., Evaluating historical candidate genes for schizophrenia. Molecular Psychiatry, 2015. 20(5): p. 555-562.

37. Narita, A., et al., Clustering by phenotype and genome-wide association study in autism. Translational Psychiatry, 2020. 10(1).

38. Pietropaolo, S., et al., Genetic-Background Modulation of Core and Variable AutisticLike Symptoms in Fmr1 Knock-Out Mice. PLoS ONE, 2011. 6(2): p. e17073. 
39. Sledziowska, M., J. Galloway, and S.J. Baudouin, Evidence for a Contribution of the Nlgn3/Cyfip1/Fmr1 Pathway in the Pathophysiology of Autism Spectrum Disorders. Neuroscience, 2020. 445: p. 31-41.

40. Mitchell, R., et al., Risk factors for the development of autism spectrum disorder in children with tuberous sclerosis complex: protocol for a systematic review. 2017. 6(1).

41. $\mathrm{Xu}, \mathrm{C}$., et al., Comprehensive literature data-mining analysis reveals a broad genetic network functionally associated with autism spectrum disorder. International Journal of Molecular Medicine, 2018.

42. Perry, B.I., et al., Common mechanisms for type 2 diabetes and psychosis: Findings from a prospective birth cohort. Schizophrenia Research, 2020. 223: p. 227-235.

43. Hackinger, S., et al., Evidence for genetic contribution to the increased risk of type 2 diabetes in schizophrenia. Translational Psychiatry, 2018. 8(1).

44. Maćkowiak, M., P. Mordalska, and K. Wędzony, Neuroligins, synapse balance and neuropsychiatric disorders. Pharmacological Reports, 2014. 66(5): p. 830-835.

45. Anney, R.J.L., et al., Meta-analysis of GWAS of over 16,000 individuals with autism spectrum disorder highlights a novel locus at 10q24.32 and a significant overlap with schizophrenia. Molecular Autism, 2017. 8(1): p. 21.

46. Bölte, S., S. Girdler, and P.B. Marschik, The contribution of environmental exposure to the etiology of autism spectrum disorder. Cellular and Molecular Life Sciences, 2019. 76(7): p. 1275-1297.

47. Misiak, B., et al., Toward a unified theory of childhood trauma and psychosis: $A$ comprehensive review of epidemiological, clinical, neuropsychological and biological findings. Neuroscience \& Biobehavioral Reviews, 2017. 75: p. 393-406.

48. Forssberg, H., Microbiome programming of brain development: implications for neurodevelopmental disorders. Developmental Medicine \& Child Neurology, 2019. 61(7): p. 744-749.

49. De Angelis, M., et al., Fecal Microbiota and Metabolome of Children with Autism and Pervasive Developmental Disorder Not Otherwise Specified. PLoS ONE, 2013. 8(10): p. e76993.

50. Strati, F., et al., New evidences on the altered gut microbiota in autism spectrum disorders. Microbiome, 2017. 5(1).

51. Kraneveld, A.D., et al., Chapter Thirteen - Gut-to-Brain Axis in Autism Spectrum Disorders: Central Role for the Microbiome, in International Review of Neurobiology, J.F. Cryan and G. Clarke, Editors. 2016, Academic Press. p. 263-287.

52. Golofast, B. and K. Vales, The connection between microbiome and schizophrenia. Neuroscience \& Biobehavioral Reviews, 2020. 108: p. 712-731.

53. Szeligowski, T., et al., The Gut Microbiome and Schizophrenia: The Current State of the Field and Clinical Applications. Frontiers in Psychiatry, 2020. 11.

54. Troisi, J., et al., Genome, Environment, Microbiome and Metabolome in Autism (GEMMA) Study Design: Biomarkers Identification for Precision Treatment and Primary Prevention of Autism Spectrum Disorders by an Integrated Multi-Omics Systems Biology Approach. Brain Sciences, 2020. 10(10): p. 743.

55. Wakefield, A., et al., RETRACTED: Ileal-lymphoid-nodular hyperplasia, non-specific colitis, and pervasive developmental disorder in children. The Lancet, 1998.

351(9103): p. 637-641. 
56. Bricker, B. and J. Justice, The Postmodern Medical Paradigm: A Case Study of AntiMMR Vaccine Arguments. Western Journal of Communication, 2019. 83(2): p. 172189.

57. Destefano, F. and W.W. Thompson, MMR vaccine and autism: an update of the scientific evidence. Expert Review of Vaccines, 2004. 3(1): p. 19-22.

58. Zimmerman, A.W., et al., Maternal antibrain antibodies in autism. Brain, Behavior, and Immunity, 2007. 21(3): p. 351-357.

59. Jones, A.L., et al., Immune dysregulation and self-reactivity in schizophrenia: Do some cases of schizophrenia have an autoimmune basis? Immunology \& Cell Biology, 2005. 83(1): p. 9-17.

60. Thion, M.S., F. Ginhoux, and S. Garel, Microglia and early brain development: An intimate journey. Science, 2018. 362(6411): p. 185-189.

61. Liao, X., et al., Microglia mediated neuroinflammation in autism spectrum disorder. Journal of Psychiatric Research, 2020. 130: p. 167-176.

62. Momtazmanesh, S., A. Zare-Shahabadi, and N. Rezaei, Cytokine Alterations in Schizophrenia: An Updated Review. Frontiers in Psychiatry, 2019. 10.

63. Wei, H., et al., Brain IL-6 elevation causes neuronal circuitry imbalances and mediates autism-like behaviors. Biochimica et Biophysica Acta (BBA) - Molecular Basis of Disease, 2012. 1822(6): p. 831-842.

64. Xie, J., et al., Immunological cytokine profiling identifies TNF- $\alpha$ as a key molecule dysregulated in autistic children. Oncotarget, 2017. 8(47): p. 82390-82398.

65. Dennison, U., et al., Schizophrenia patients with a history of childhood trauma have a pro-inflammatory phenotype. Psychological Medicine, 2012. 42(9): p. 1865-1871.

66. Ozaki, K., et al., Maternal immune activation induces sustained changes in fetal microglia motility. Scientific Reports, 2020. 10(1).

67. Moradi, K., et al., The interplay between gut microbiota and autism spectrum disorders: A focus on immunological pathways. Progress in NeuroPsychopharmacology and Biological Psychiatry, 2021. 106: p. 110091.

68. Theoharides, T.C., M. Kavalioti, and I. Tsilioni, Mast Cells, Stress, Fear and Autism Spectrum Disorder. International Journal of Molecular Sciences, 2019. 20(15): p. 3611.

69. Sellgren, C.M., et al., Increased synapse elimination by microglia in schizophrenia patient-derived models of synaptic pruning. Nature Neuroscience, 2019. 22(3): p. 374-385.

70. Rubenstein, J.L.R. and M.M. Merzenich, Model of autism: increased ratio of excitation/inhibition in key neural systems. Genes, Brain and Behavior, 2003. 2(5): p. 255-267.

71. Marotta, R., et al., The Neurochemistry of Autism. Brain Sciences, 2020. 10(3): p. 163.

72. Stahl, S.M., Beyond the dopamine hypothesis of schizophrenia to three neural networks of psychosis: dopamine, serotonin, and glutamate. CNS Spectrums, 2018. 23(3): p. 187-191.

73. Winter, C., et al., Dopamine and serotonin levels following prenatal viral infection in mouse-Implications for psychiatric disorders such as schizophrenia and autism. European Neuropsychopharmacology, 2008. 18(10): p. 712-716.

74. Canitano, R., Epilepsy in autism spectrum disorders. European Child \& Adolescent Psychiatry, 2007. 16(1): p. 61-66. 
75. Josephson, C.B. and N. Jetté, Psychiatric comorbidities in epilepsy. International Review of Psychiatry, 2017. 29(5): p. 409-424.

76. Joëls, M., Stress, the hippocampus, and epilepsy. Epilepsia, 2009. 50(4): p. 586-597.

77. Citri, A. and R.C. Malenka, Synaptic Plasticity: Multiple Forms, Functions, and Mechanisms. Neuropsychopharmacology, 2008. 33(1): p. 18-41.

78. Pelkey, K.A. and C.J. McBain, Ionotropic Glutamate Receptors in Synaptic Plasticity. Humana Press. p. 179-246.

79. Ba, W., J. Van Der Raadt, and N. Nadif Kasri, Rho GTPase signaling at the synapse: Implications for intellectual disability. Experimental Cell Research, 2013. 319(15): p. 2368-2374.

80. Pop, A.S., et al., Fragile $X$ syndrome: a preclinical review on metabotropic glutamate receptor 5 (mGluR5) antagonists and drug development. Psychopharmacology, 2014. 231(6): p. 1217-1226.

81. Kirmse, K., et al., GABA depolarizes immature neurons and inhibits network activity in the neonatal neocortex in vivo. Nature Communications, 2015. 6(1): p. 7750.

82. Rees, E., et al., De novo mutations identified by exome sequencing implicate rare missense variants in SLC6A1 in schizophrenia. Nature Neuroscience, 2020. 23(2): p. 179-184.

83. Graf, E.R., et al., Neurexins Induce Differentiation of GABA and Glutamate Postsynaptic Specializations via Neuroligins. Cell, 2004. 119(7): p. 1013-1026.

84. Hines, R.M., et al., Synaptic Imbalance, Stereotypies, and Impaired Social Interactions in Mice with Altered Neuroligin 2 Expression. 2008. 28(24): p. 6055-6067.

85. Cellot, G. and E. Cherubini, GABAergic Signaling as Therapeutic Target for Autism Spectrum Disorders. Frontiers in Pediatrics, 2014. 2.

86. Anand, K.S. and V. Dhikav, Hippocampus in health and disease: An overview. Annals of Indian Academy of Neurology, 2012. 15(4): p. 239-246.

87. Patrich, E., et al., Maternal immune activation produces neonatal excitability defects in offspring hippocampal neurons from pregnant rats treated with poly I:C. Scientific Reports, 2016. 6(1): p. 19106.

88. Heckers, S. and C. Konradi, Hippocampal neurons in schizophrenia. Journal of Neural Transmission, 2002. 109(5-6): p. 891-905.

89. D'Mello, A.M. and C.J. Stoodley, Cerebro-cerebellar circuits in autism spectrum disorder. Frontiers in Neuroscience, 2015. 9.

90. Belmonte, M.K., Autism and Abnormal Development of Brain Connectivity. Journal of Neuroscience, 2004. 24(42): p. 9228-9231.

91. Lipska, B.K. and D.R. Weinberger, A neurodevelopmental model of schizophrenia: Neonatal disconnection of the hippocampus. Neurotoxicity Research, 2002. 4(5-6): p. 469-475.

92. Dajani, D.R. and L.Q. Uddin, Local brain connectivity across development in autism spectrum disorder: A cross-sectional investigation. Autism Research, 2016. 9(1): p. 43-54.

93. Kozhemiako, N., et al., Alterations in Local Connectivity and Their Developmental Trajectories in Autism Spectrum Disorder: Does Being Female Matter? Cerebral Cortex, 2020. 30(9): p. 5166-5179.

94. Ruzzo, E.K., et al., Inherited and De Novo Genetic Risk for Autism Impacts Shared Networks. Cell, 2019. 178(4): p. 850-866.e26. 
95. McCann, K.E., et al., Novel role for mineralocorticoid receptors in control of a neuronal phenotype. Molecular Psychiatry, 2019.

96. Ehninger, D. and A.J. Silva, Rapamycin for treating Tuberous sclerosis and Autism spectrum disorders. Trends in Molecular Medicine, 2011. 17(2): p. 78-87.

97. Takei, N. and H. Nawa, mTOR signaling and its roles in normal and abnormal brain development. Frontiers in Molecular Neuroscience, 2014. 7.

98. Zaretsky, M.V., et al., Transfer of Inflammatory Cytokines Across the Placenta. Obstetrics \& Gynecology, 2004. 103(3).

99. Hsiao, E.Y. and P.H. Patterson, Activation of the maternal immune system induces endocrine changes in the placenta via IL-6. Brain, Behavior, and Immunity, 2011. 25(4): p. 604-615.

100. Budday, S., P. Steinmann, and E. Kuhl, Physical biology of human brain development. Frontiers in Cellular Neuroscience, 2015. 9.

101. Brown, A.S., et al., Prenatal rubella, premorbid abnormalities, and adult schizophrenia. Biological Psychiatry, 2001. 49(6): p. 473-486.

102. Brown, A.S., et al., Maternal Exposure to Toxoplasmosis and Risk of Schizophrenia in Adult Offspring. American Journal of Psychiatry, 2005. 162(4): p. 767-773.

103. Patterson, P.H., Immune involvement in schizophrenia and autism: Etiology, pathology and animal models. Behavioural Brain Research, 2009. 204(2): p. 313-321.

104. McGrath, J.J., et al., Schizophrenia and the influenza epidemics of 1954, 1957 and 1959: A southern hemisphere study. Schizophrenia Research, 1994. 14(1): p. 1-8.

105. Shi, L., et al., Maternal Influenza Infection Causes Marked Behavioral and Pharmacological Changes in the Offspring. The Journal of Neuroscience, 2003. 23(1): p. 297-302.

106. Zerbo, O., et al., Is Maternal Influenza or Fever During Pregnancy Associated with Autism or Developmental Delays? Results from the CHARGE (CHildhood Autism Risks from Genetics and Environment) Study. Journal of Autism and Developmental Disorders, 2013. 43(1): p. 25-33.

107. Atladóttir, H.Ó., et al., Maternal Infection Requiring Hospitalization During Pregnancy and Autism Spectrum Disorders. 2010. 40(12): p. 1423-1430.

108. Abdallah, M.W., et al., Amniotic fluid chemokines and autism spectrum disorders: An exploratory study utilizing a Danish Historic Birth Cohort. Brain, Behavior, and Immunity, 2012. 26(1): p. 170-176.

109. Zhang, J.-M. and J. An, Cytokines, Inflammation, and Pain. International Anesthesiology Clinics, 2007. 45(2): p. 27-37.

110. Jones, K.L., et al., Autism with intellectual disability is associated with increased levels of maternal cytokines and chemokines during gestation. Molecular Psychiatry, 2017. 22(2): p. 273-279.

111. Gładysz, D., A. Krzywdzińska, and K.K. Hozyasz, Immune Abnormalities in Autism Spectrum Disorder-Could They Hold Promise for Causative Treatment? Molecular Neurobiology, 2018.

112. Goines, P.E., et al., Increased midgestational IFN- $\gamma, I L-4$ and IL-5 in women bearing a child with autism: A case-control study. Molecular Autism, 2011. 2(1): p. 13.

113. Choi, G.B., et al., The maternal interleukin-17a pathway in mice promotes autism-like phenotypes in offspring. Science, 2016. 351(6276): p. 933-939.

114. Patterson, P.H., et al., Maternal Immune Activation, Cytokines and Autism. 2008, Humana Press. p. 289-307. 
115. Dahlgren, J., et al., Interleukin-6 in the Maternal Circulation Reaches the Rat Fetus in Mid-gestation. Pediatric Research, 2006. 60(2): p. 147-151.

116. Tsukada, T., et al., Molecular mechanisms underlying the models of neurodevelopmental disorders in maternal immune activation relevant to the placenta. Congenital Anomalies, 2019. 59(3): p. 81-87.

117. Wu, W.-L., et al., The placental interleukin-6 signaling controls fetal brain development and behavior. Brain, Behavior, and Immunity, 2017. 62: p. 11-23.

118. Hu, B., S. Huang, and L. Yin, The cytokine storm and COVID-19. Journal of Medical Virology, 2021. 93(1): p. 250-256.

119. Lima, M.E.D.S., L.C.M. Barros, and G.F. Aragão, Could autism spectrum disorders be a risk factor for COVID-19? Medical Hypotheses, 2020. 144: p. 109899.

120. Matsuura, M., Structural Modifications of Bacterial Lipopolysaccharide that Facilitate Gram-Negative Bacteria Evasion of Host Innate Immunity. Frontiers in Immunology, 2013. 4.

121. Fortier, M.-E., et al., The viral mimic, polyinosinic:polycytidylic acid, induces fever in rats via an interleukin-1-dependent mechanism. American Journal of PhysiologyRegulatory, Integrative and Comparative Physiology, 2004. 287(4): p. R759-R766.

122. Reisinger, S., et al., The Poly(I:C)-induced maternal immune activation model in preclinical neuropsychiatric drug discovery. Pharmacology \& Therapeutics, 2015. 149: p. 213-226.

123. Carlezon, W.A., et al., Maternal and early postnatal immune activation produce sexspecific effects on autism-like behaviors and neuroimmune function in mice. Scientific Reports, 2019. 9(1).

124. Careaga, M., T. Murai, and M.D. Bauman, Maternal Immune Activation and Autism Spectrum Disorder: From Rodents to Nonhuman and Human Primates. Biological Psychiatry, 2017. 81(5): p. 391-401.

125. Kentner, A.C., et al., Maternal immune activation: reporting guidelines to improve the rigor, reproducibility, and transparency of the model. Neuropsychopharmacology, 2019. 44(2): p. 245-258.

126. Boulanger-Bertolus, J., C. Pancaro, and G.A. Mashour, Increasing Role of Maternal Immune Activation in Neurodevelopmental Disorders. Frontiers in Behavioral Neuroscience, 2018. 12.

127. Lombardo, M.V., et al., Maternal immune activation dysregulation of the fetal brain transcriptome and relevance to the pathophysiology of autism spectrum disorder. Molecular Psychiatry, 2018. 23(4): p. 1001-1013.

128. Daubert, E.A. and B.G. Condron, Serotonin: a regulator of neuronal morphology and circuitry. Trends in Neurosciences, 2010. 33(9): p. 424-434.

129. Sundström, E., et al., Neurochemical differentiation of human bulbospinal monoaminergic neurons during the first trimester. Brain Res Dev Brain Res, 1993. 75(1): p. 1-12.

130. Sahu, A., et al., The 5-Hydroxytryptamine signaling map: an overview of serotoninserotonin receptor mediated signaling network. Journal of Cell Communication and Signaling, 2018. 12(4): p. 731-735.

131. Lesch, K.-P. and J. Waider, Serotonin in the Modulation of Neural Plasticity and Networks: Implications for Neurodevelopmental Disorders. Neuron, 2012. 76(1): p. 175-191. 
132. Brummelte, S., et al., Developmental changes in serotonin signaling: Implications for early brain function, behavior and adaptation. Neuroscience, 2017. 342: p. 212-231.

133. Gordon, J.A. and R. Hen, The Serotonergic System and Anxiety. NeuroMolecular Medicine, 2004. 5(1): p. 027-040.

134. Heisler, L.K., et al., Serotonin Activates the Hypothalamic-Pituitary-Adrenal Axis via Serotonin 2C Receptor Stimulation. Journal of Neuroscience, 2007. 27(26): p. 69566964.

135. Canli, T. and K.-P. Lesch, Long story short: the serotonin transporter in emotion regulation and social cognition. Nature Neuroscience, 2007. 10(9): p. 1103-1109.

136. Schloss, P. and D.C. Williams, The serotonin transporter: a primary target for antidepressant drugs. J Psychopharmacol, 1998. 12(2): p. 115-21.

137. McVey Neufeld, K.-A., et al., Oral selective serotonin reuptake inhibitors activate vagus nerve dependent gut-brain signalling. Scientific Reports, 2019. 9(1).

138. Hrovatin, K., T. Kunej, and V. Dolžan, Genetic variability of serotonin pathway associated with schizophrenia onset, progression, and treatment. American Journal of Medical Genetics Part B: Neuropsychiatric Genetics, 2020. 183(2): p. 113-127.

139. Aghajanian, G.K. and G.J. Marek, Serotonin model of schizophrenia: emerging role of glutamate mechanisms. Brain Research Reviews, 2000. 31(2): p. 302-312.

140. Nasrallah, H.A., R. Fedora, and R. Morton, Successful treatment of clozapinenonresponsive refractory hallucinations and delusions with pimavanserin, a serotonin 5HT-2A receptor inverse agonist. Schizophrenia Research, 2019. 208: p. 217-220.

141. Tanaka, M., et al., Brain hyperserotonemia causes autism-relevant social deficits in mice. Molecular Autism, 2018. 9(1).

142. Croonenberghs, J., et al., Central serotonergic hypofunction in autism: results of the 5-hydroxy-tryptophan challenge test. Neuro Endocrinol Lett, 2007. 28(4): p. 449-55.

143. Andersson, M., et al., Serotonin transporter availability in adults with autism - a positron emission tomography study. Molecular Psychiatry, 2020.

144. De Neve, J.-E., Functional polymorphism (5-HTTLPR) in the serotonin transporter gene is associated with subjective well-being: evidence from a US nationally representative sample. Journal of Human Genetics, 2011. 56(6): p. 456-459.

145. Gelernter, J., et al., Population studies of polymorphisms of the serotonin transporter protein gene. Am J Med Genet, 1999. 88(1): p. 61-6.

146. Palma-Gudiel, H. and L. Fañanás, An integrative review of methylation at the serotonin transporter gene and its dialogue with environmental risk factors, psychopathology and 5-HTTLPR. Neuroscience \& Biobehavioral Reviews, 2017. 72: p. 190-209.

147. Border, R., et al., No Support for Historical Candidate Gene or Candidate Gene-byInteraction Hypotheses for Major Depression Across Multiple Large Samples. American Journal of Psychiatry, 2019. 176(5): p. 376-387.

148. Fratelli, C., et al., 5HTTLPR Genetic Variant and Major Depressive Disorder: A Review. Genes, 2020. 11(11): p. 1260.

149. Tetlow, A.L. and F. Tamanoi, Chapter One - The Ras Superfamily G-Proteins, in The Enzymes, F. Tamanoi, Editor. 2013, Academic Press. p. 1-14.

150. Linseman, D.A. and F.A. Loucks Diverse roles of Rho family GTPases in neuronal development, survival, and death. Frontiers in bioscience : a journal and virtual library, 2008. 13, 657-676 DOI: 10.2741/2710. 
151. Hall, A. and G. Lalli, Rho and Ras GTPases in Axon Growth, Guidance, and Branching. Cold Spring Harbor Perspectives in Biology, 2010. 2(2): p. a001818-a001818.

152. Hall, A., Rho GTPases and the Actin Cytoskeleton. Science, 1998. 279(5350): p. 509514.

153. Tsukada, Y., et al., Quantification of Local Morphodynamics and Local GTPase Activity by Edge Evolution Tracking. 2008. 4(11): p. e1000223.

154. Kuhn, T.B., M.D. Brown, and J.R. Bamburg, Rac1-dependent actin filament organization in growth cones is necessary for B1-integrin-mediated advance but not for growth on poly-D-lysine. Journal of Neurobiology, 1998. 37(4): p. 524-540.

155. Hua, Z.L., F.E. Emiliani, and J. Nathans, Rac1 plays an essential role in axon growth and guidance and in neuronal survival in the central and peripheral nervous systems. 2015. 10(1).

156. Bustelo, X.R., et al., Rac-ing to the plasma membrane. Small GTPases, 2012. 3(1): p. 60-66.

157. Pyronneau, A., et al., Aberrant Rac1-cofilin signaling mediates defects in dendritic spines, synaptic function, and sensory perception in fragile $X$ syndrome. Science Signaling, 2017. 10(504): p. eaan0852.

158. Martinez, L.A. and M.V. Tejada-Simon, Pharmacological inactivation of the small GTPase Rac1 impairs long-term plasticity in the mouse hippocampus.

Neuropharmacology, 2011. 61(1-2): p. 305-312.

159. Parnell, E., et al., KALRN: A central regulator of synaptic function and synaptopathies. Gene, 2021. 768: p. 145306.

160. Ma, X.-M., et al., Kalirin, a Multifunctional Rho Guanine Nucleotide Exchange Factor, Is Necessary for Maintenance of Hippocampal Pyramidal Neuron Dendrites and Dendritic Spines. The Journal of Neuroscience, 2003. 23(33): p. 10593-10603.

161. Ma, X.M., et al., Kalirin-7 is required for synaptic structure and function. J Neurosci, 2008. 28(47): p. 12368-82.

162. Sommer, J.E. and E.C. Budreck, Kalirin-7: Linking Spine Plasticity and Behavior. Journal of Neuroscience, 2009. 29(17): p. 5367-5369.

163. Herring, B.E. and R.A. Nicoll, Kalirin and Trio proteins serve critical roles in excitatory synaptic transmission and LTP. Proceedings of the National Academy of Sciences, 2016. 113(8): p. 2264-2269.

164. Hill, J.J., T. Hashimoto, and D.A. Lewis, Molecular mechanisms contributing to dendritic spine alterations in the prefrontal cortex of subjects with schizophrenia. Molecular Psychiatry, 2006. 11(6): p. 557-566.

165. Russell, T.A., et al., A Schizophrenia-Linked KALRN Coding Variant Alters Neuron Morphology, Protein Function, and Transcript Stability. Biological Psychiatry, 2018. 83(6): p. 499-508.

166. Hayashi-Takagi, A., et al., Disrupted-in-Schizophrenia 1 (DISC1) regulates spines of the glutamate synapse via Rac1. Nature Neuroscience, 2010. 13(3): p. 327-332.

167. Owczarek, S., M.L. Bang, and V. Berezin, Neurexin-Neuroligin Synaptic Complex Regulates Schizophrenia-Related DISC1/Kal-7/Rac1 "Signalosome". Neural Plasticity, 2015. 2015: p. 167308.

168. Chubb, J.E., et al., The DISC locus in psychiatric illness. Molecular Psychiatry, 2008. 13(1): p. 36-64.

169. St Clair, D., et al., Association within a family of a balanced autosomal translocation with major mental illness. The Lancet, 1990. 336(8706): p. 13-16. 
170. Naghavi-Gargari, B., et al., Significant increasing of DISC2 long non-coding RNA expression as a potential biomarker in bipolar disorder. Neuroscience Letters, 2019. 696: p. 206-211.

171. Kilpinen, H., et al., Association of DISC1 with autism and Asperger syndrome. Molecular Psychiatry, 2008. 13(2): p. 187-196.

172. Lipina, T.V., et al., Maternal Immune Activation during Gestation Interacts with Disc1 Point Mutation to Exacerbate Schizophrenia-Related Behaviors in Mice. Journal of Neuroscience, 2013. 33(18): p. 7654-7666.

173. Mendoza, C., et al., Toll-like Receptor 3 Activation Affects Serotonin Transporter Activity and Expression in Human Enterocyte-like Caco-2 Cells. Cellular Physiology and Biochemistry, 2012. 30(1): p. 187-198.

174. Coiro, P. and D.D. Pollak, Sex and gender bias in the experimental neurosciences: the case of the maternal immune activation model. Translational Psychiatry, 2019. 9(1).

175. Homberg, J.R., et al., Characterization of the serotonin transporter knockout rat: $A$ selective change in the functioning of the serotonergic system. Neuroscience, 2007. 146(4): p. 1662-1676.

176. Balcombe, J.P., Laboratory environments and rodents' behavioural needs: a review. Laboratory Animals, 2006. 40(3): p. 217-235.

177. Festing, M.F.W. and D.G. Altman, Guidelines for the Design and Statistical Analysis of Experiments Using Laboratory Animals. ILAR Journal, 2002. 43(4): p. 244-258.

178. Estes, M.L., et al., Enhancing rigor and reproducibility in maternal immune activation models: practical considerations and predicting resilience and susceptibility using baseline immune responsiveness before pregnancy. 2019, Cold Spring Harbor Laboratory.

179. Missault, S., et al., The risk for behavioural deficits is determined by the maternal immune response to prenatal immune challenge in a neurodevelopmental model. Brain, Behavior, and Immunity, 2014. 42: p. 138-146.

180. Careaga, M., et al., Variability in PolyIC induced immune response: Implications for preclinical maternal immune activation models. Journal of Neuroimmunology, 2018. 323: p. 87-93.

181. Long, N.C., A.J. Vander, and M.J. Kluger, Stress-induced rise of body temperature in rats is the same in warm and cool environments. Physiology \& Behavior, 1990. 47(4): p. 773-775.

182. Willadsen, M., et al., Reduced emission of alarm $22-\mathrm{kHz}$ ultrasonic vocalizations during fear conditioning in rats lacking the serotonin transporter. Progress in NeuroPsychopharmacology and Biological Psychiatry, 2020: p. 110072.

183. Dimitsantos, E., et al., Litter size affects emotionality in adult male rats. Physiology \& Behavior, 2007. 92(4): p. 708-716.

184. Spencer, S.J. and A. Tilbrook, Neonatal overfeeding alters adult anxiety and stress responsiveness. Psychoneuroendocrinology, 2009. 34(8): p. 1133-1143.

185. Murray, K.N., et al., Evolution of a maternal immune activation (mIA) model in rats: Early developmental effects. Brain, Behavior, and Immunity, 2019. 75: p. 48-59.

186. Haida, O., et al., Sex-dependent behavioral deficits and neuropathology in a maternal immune activation model of autism. Translational Psychiatry, 2019. 9(1).

187. Laviola, G. and E. Alleva, Sibling effects on the behavior of infant mouse litters (Mus domesticus). J Comp Psychol, 1995. 109(1): p. 68-75. 
188. Namikas, J. and F. Wehmer, Gender composition of the litter affects behavior of male mice. Behavioral Biology, 1978. 23(2): p. 219-224.

189. Lazic, S.E. and L. Essioux, Improving basic and translational science by accounting for litter-to-litter variation in animal models. BMC Neurosci, 2013. 14: p. 37.

190. Even, M.D., M.G. Dhar, and F.S. vom Saal, Transport of steroids between fetuses via amniotic fluid in relation to the intrauterine position phenomenon in rats. J Reprod Fertil, 1992. 96(2): p. 709-16.

191. Anderson, J.W., The Production of Ultrasonic Sounds by Laboratory Rats and Other Mammals. Science, 1954. 119(3101): p. 808-809.

192. Simola, N., Rat Ultrasonic Vocalizations and Behavioral Neuropharmacology: From the Screening of Drugs to the Study of Disease. Current Neuropharmacology, 2015. 13(2): p. 164-179.

193. Malkova, N.V., et al., Maternal immune activation yields offspring displaying mouse versions of the three core symptoms of autism. Brain, Behavior, and Immunity, 2012. 26(4): p. 607-616.

194. Mogi, K., et al., Mutual mother-infant recognition in mice: The role of pup ultrasonic vocalizations. Behavioural Brain Research, 2017. 325: p. 138-146.

195. Yin, X., et al., Maternal Deprivation Influences Pup Ultrasonic Vocalizations of C57BL/6J Mice. PLOS ONE, 2016. 11(8): p. e0160409.

196. Kromkhun, P., et al., Quantitative and qualitative analysis of rat pup ultrasonic vocalization sounds induced by a hypothermic stimulus. Laboratory Animal Research, 2013. 29(2): p. 77.

197. Isobe, A. and M. Kawaguchi, Relationship between motor function and ultrasonic vocalizations induced by maternal separation in rat pups. Journal of Veterinary Medical Science, 2019. 81(2): p. 287-293.

198. Rieger, M.A. and J.D. Dougherty, Analysis of within Subjects Variability in Mouse Ultrasonic Vocalization: Pups Exhibit Inconsistent, State-Like Patterns of Call Production. Frontiers in Behavioral Neuroscience, 2016. 10.

199. Holmes, A., D.L. Murphy, and J.N. Crawley, Abnormal behavioral phenotypes of serotonin transporter knockout mice: parallels with human anxiety and depression. Biological Psychiatry, 2003. 54(10): p. 953-959.

200. Chan, J.S.W., et al., The Serotonin Transporter Plays an Important Role in Male Sexual Behavior: A Study in Serotonin Transporter Knockout Rats. The Journal of Sexual Medicine, 2011. 8(1): p. 97-108.

201. Golebiowska, J., et al., Serotonin transporter deficiency alters socioemotional ultrasonic communication in rats. Scientific Reports, 2019. 9(1).

202. Houwing, D.J., et al., Subjecting Dams to Early Life Stress and Perinatal Fluoxetine Treatment Differentially Alters Social Behavior in Young and Adult Rat Offspring. Frontiers in Neuroscience, 2019. 13.

203. Mosienko, V., et al., Reduced isolation-induced pup ultrasonic communication in mouse pups lacking brain serotonin. Molecular Autism, 2015. 6(1).

204. Program, B.R., Raven Pro: interactive sound analysis software (Version 1.5). 2014, Cornell Lab of Ornithology Ithaca, NY, USA.

205. Vogel, A.P., A. Tsanas, and M.L. Scattoni, Quantifying ultrasonic mouse vocalizations using acoustic analysis in a supervised statistical machine learning framework. Scientific Reports, 2019. 9(1). 
206. Coffey, K.R., R.G. Marx, and J.F. Neumaier, DeepSqueak: a deep learning-based system for detection and analysis of ultrasonic vocalizations.

Neuropsychopharmacology, 2019. 44(5): p. 859-868.

207. Johnson, A., et al., Changes in Rat 50-kHz Ultrasonic Vocalizations During Dopamine Denervation and Aging: Relevance to Neurodegeneration. Current Neuropharmacology, 2015. 13(2): p. 211-219.

208. Harrison, X.A., et al., A brief introduction to mixed effects modelling and multi-model inference in ecology. PeerJ, 2018. 6: p. e4794.

209. Rao, X., et al., An improvement of the $2^{\wedge}$ (-delta delta CT) method for quantitative real-time polymerase chain reaction data analysis. Biostat Bioinforma Biomath, 2013. 3(3): p. 71-85.

210. Bustin, S.A., et al., The MIQE Guidelines: Minimum Information for Publication of Quantitative Real-Time PCR Experiments. Clinical Chemistry, 2009. 55(4): p. 611-622.

211. Boccara, C.N., et al., A three-plane architectonic atlas of the rat hippocampal region. Hippocampus, 2015. 25(7): p. 838-57.

212. Iwano, T., et al., Prox1 postmitotically defines dentate gyrus cells by specifying granule cell identity over CA3 pyramidal cell fate in the hippocampus. Development, 2012. 139(16): p. 3051-3062.

213. Wegiel, J., et al., The neuropathology of autism: defects of neurogenesis and neuronal migration, and dysplastic changes. Acta Neuropathologica, 2010. 119(6): p. 755-770.

214. Lavado, A. and G. Oliver, Prox1 expression patterns in the developing and adult murine brain. Developmental Dynamics, 2007. 236(2): p. 518-524.

215. Veselenak, R.L., et al., Development and Utilization of a Custom PCR Array Workflow: Analysis of Gene Expression in Mycoplasma genitalium and Guinea Pig (Cavia porcellus). Molecular Biotechnology, 2015. 57(2): p. 172-183.

216. Schielzeth, H., et al., Robustness of linear mixed-effects models to violations of distributional assumptions. Methods in Ecology and Evolution, 2020. 11(9): p. 11411152.

217. Jacqmin-Gadda, H., et al., Robustness of the linear mixed model to misspecified error distribution. Computational Statistics \& Data Analysis, 2007. 51(10): p. 5142-5154.

218. Müller, S., J.L. Scealy, and A.H. Welsh, Model Selection in Linear Mixed Models. Statistical Science, 2013. 28(2): p. 135-167.

219. Pryce, C.R., et al., Comparison of the effects of early handling and early deprivation on conditioned stimulus, context, and spatial learning and memory in adult rats. Behav Neurosci, 2003. 117(5): p. 883-93.

220. Justice, M.J. and P. Dhillon, Using the mouse to model human disease: increasing validity and reproducibility. Disease Models \& Mechanisms, 2016. 9(2): p. 101-103.

221. Servadio, M., L.J. Vanderschuren, and V. Trezza, Modeling autism-relevant behavioral phenotypes in rats and mice: Do 'autistic' rodents exist? Behav Pharmacol, 2015. 26(6): p. 522-40.

222. Cast, T.P., et al., An Autism-Associated Mutation Impairs Neuroligin-4 Glycosylation and Enhances Excitatory Synaptic Transmission in Human Neurons. The Journal of Neuroscience, 2021. 41(3): p. 392. 


\section{Appendix 1. Parental Genotyping}

\section{Figure A1.1: Parental Genotyping Results}

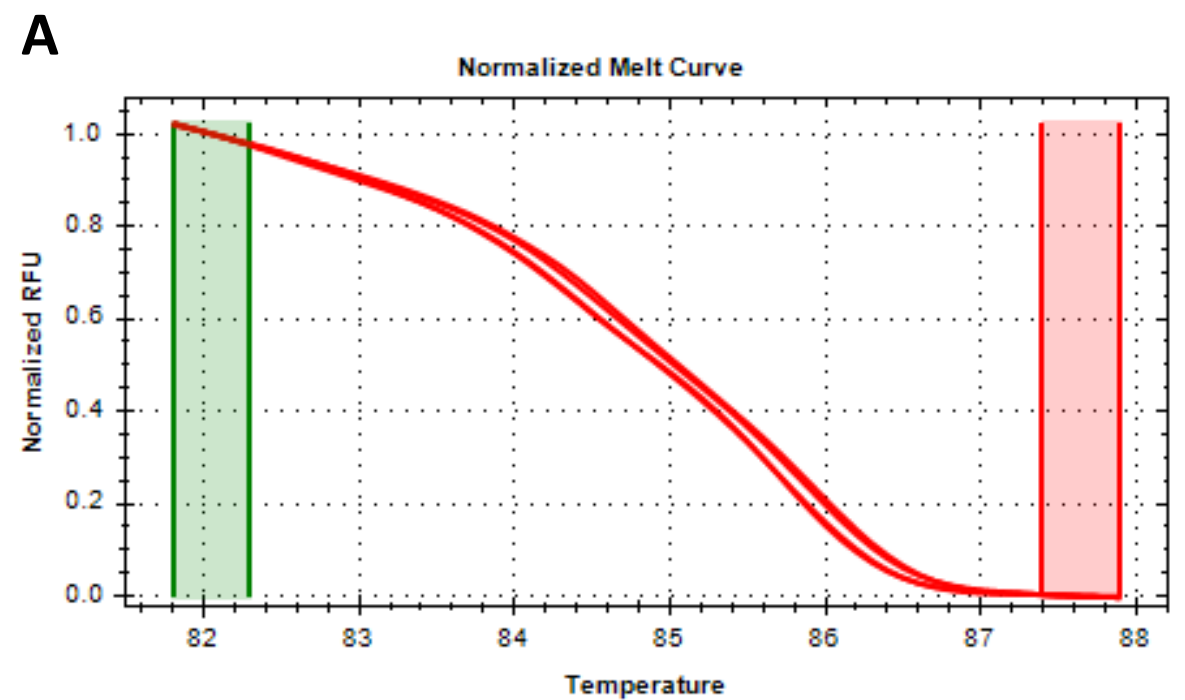

B

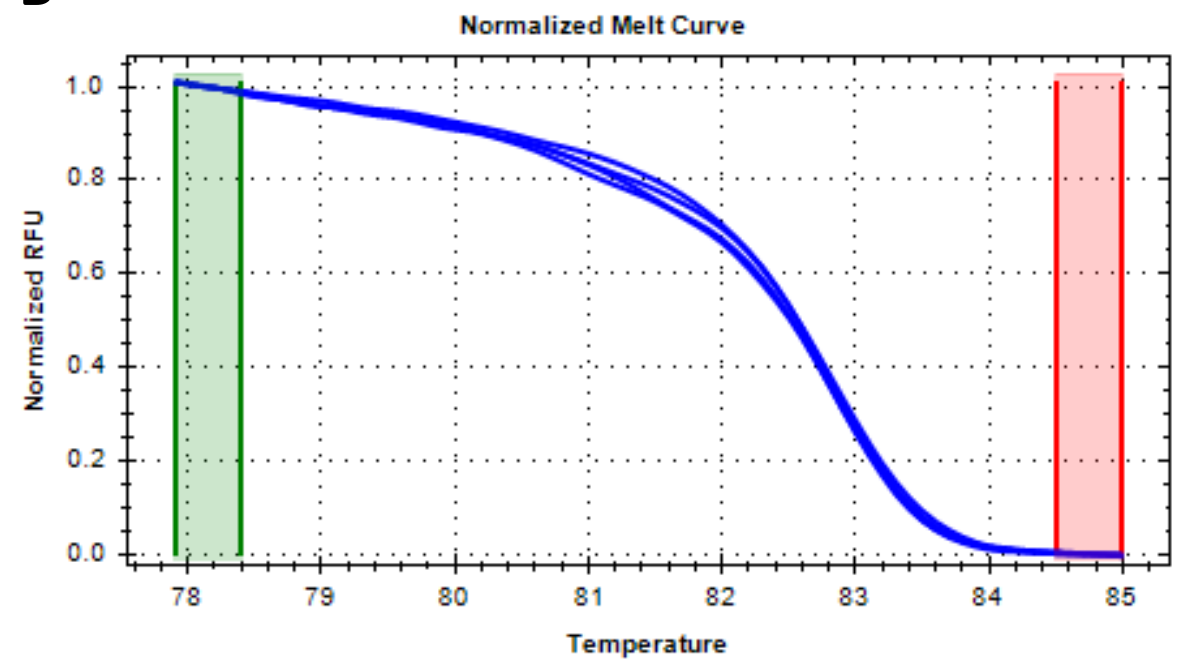

Figure A1.1: Genotyping performed on breeding animals confirmed previous genotyping information. A sample offemales $(n=5)$ and males $(n=4)$ had ear punches taken for DNA isolation and re-genotyping. Each set contained animals which had parented litters with 100\% genotype identification, and parents of litters with pups of unknown genotype. A) Breeding females assessed by HRMA using SERT1 primers identified all sampled females as the same genotype and as SERT HETS (references not shown). B) All breeding males tested using SERT3 primers amplified as the same genotype, SERT WT (references not shown). No unusual amplification was observed in parents of unidentified offspring or across primer sets, suggesting results from unidentified pups was not due to a genetically inherited trait (such as an abnormal allelic variant or a mutation causing the presence of a secondary priming loci and the replication of multiple PCR products). 


\section{Appendix 2. Linear Mixed Modelling}

Table A2.1: F-statistics for USV Study Using Linear Mixed Modelling

\begin{tabular}{|c|c|c|c|c|c|c|c|c|c|}
\hline & \multicolumn{8}{|c|}{ Main and Interaction Effects } \\
\hline \multirow{5}{*}{ 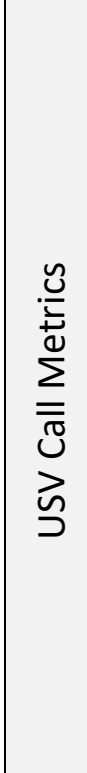 } & Number of Calls & 431.59 & 0.30 & 7.22 & 1.72 & 2.00 & 0.28 & 0.64 & 2.62 \\
\hline & Time Calling & 368.29 & 1.68 & 4.76 & 1.04 & 0.10 & 0.09 & 0.05 & 0.57 \\
\hline & Average Call Length & $2,398.62$ & 0.95 & $<0.01$ & 0.15 & 1.42 & 0.53 & 0.87 & 0.19 \\
\hline & $\begin{array}{l}\text { Average Principal } \\
\text { Frequency }\end{array}$ & $17,469.95$ & 0.05 & 9.91 & 0.05 & 0.33 & $<0.01$ & $<0.01$ & 0.47 \\
\hline & $\begin{array}{l}\text { Average Frequency } \\
\text { Change }\end{array}$ & 912.60 & 0.63 & 14.03 & 3.49 & 1.27 & 0.06 & 1.07 & 0.18 \\
\hline
\end{tabular}

Table A2.1: F-statistics for all ANOVAs run using linear mixed modelling of USV call measurements. This table corresponds to ANOVA generated $p$ values presented in Table 4.2. 
Table A2.2: F-statistics for Gene Expression Study Using Linear Mixed Modelling

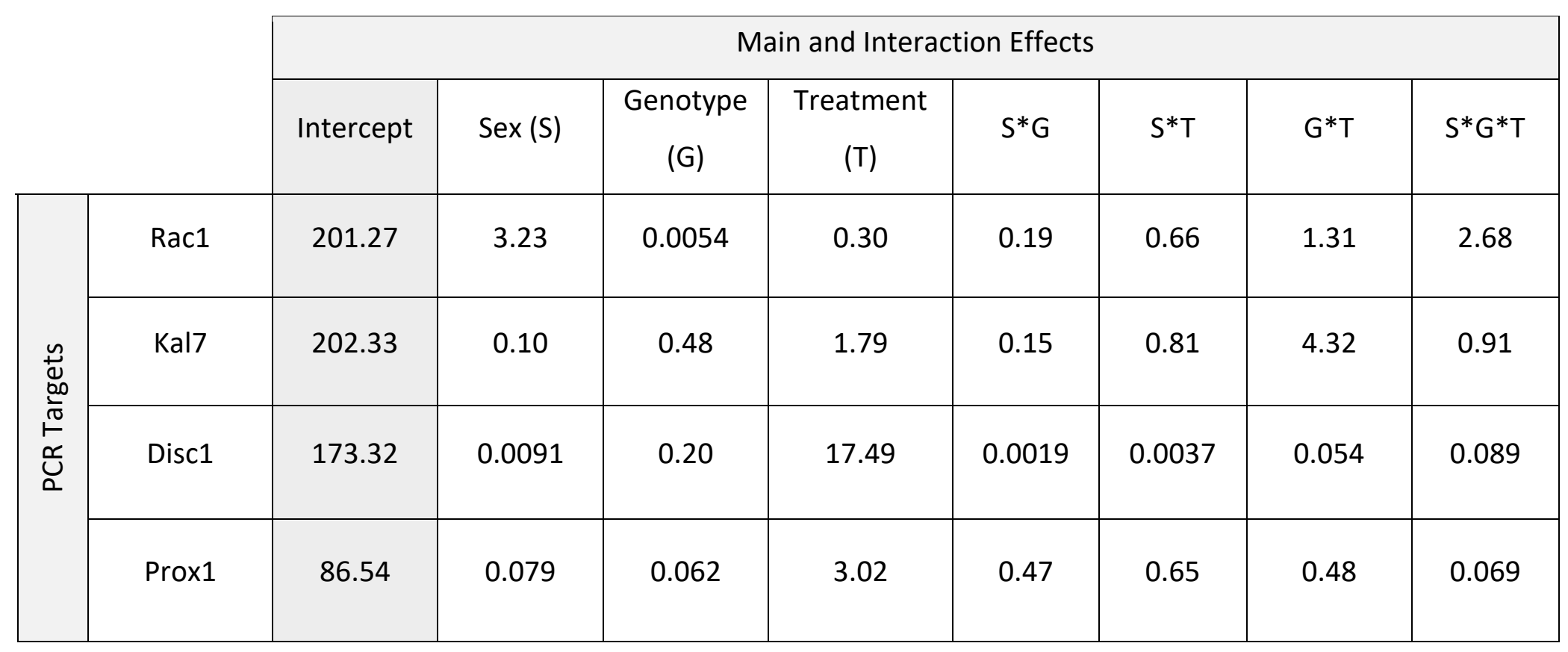

Table A2.2: F-statistics for all ANOVAs run using linear mixed modelling of fold expression data produced from RT-qPCR experiments. This table corresponds to ANOVA generated $p$ values presented in Table 5.1. 


\title{
Appendix 3: Non-Significant USV Pairwise Comparisons
}

\author{
Figure A3.1: Non-Significant Genotype Comparisons
}
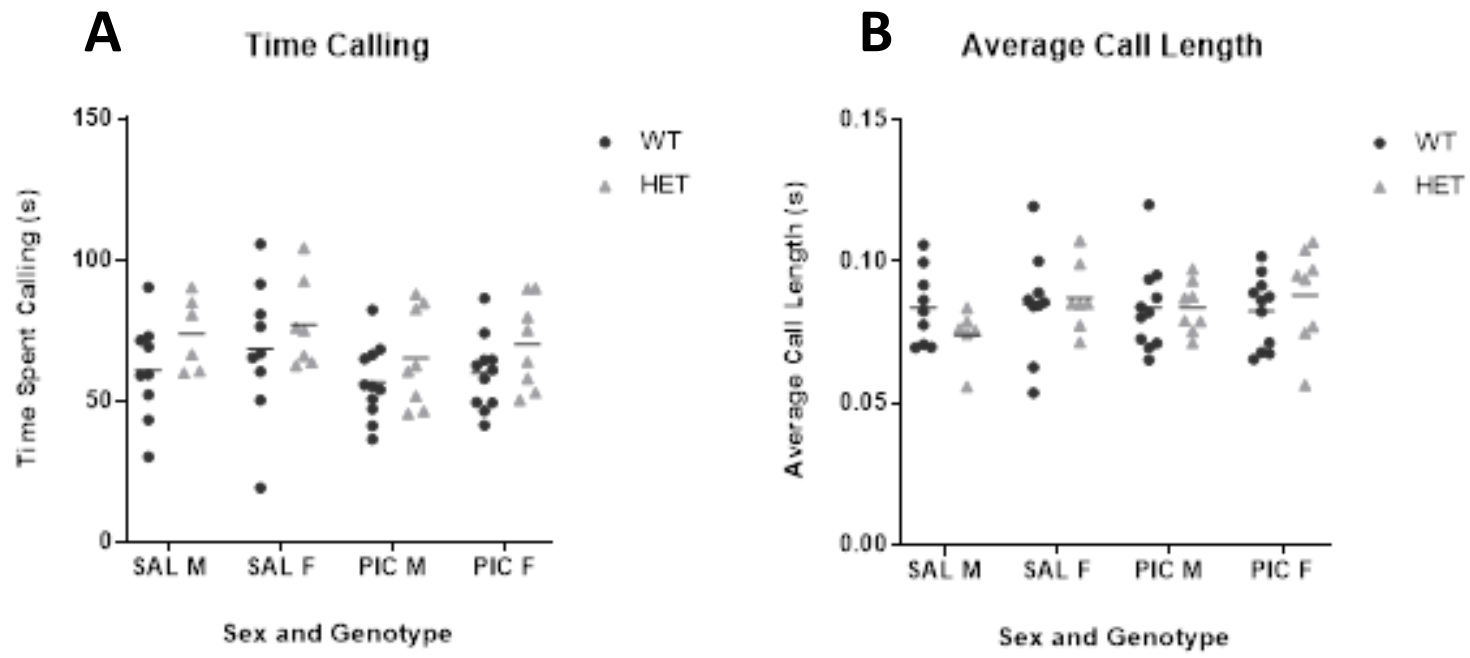

Figure A3.1: Pairwise comparisons in Time Calling (A) and Average Call Length (B) are presented above. These did not meet statistical significance in any sex/treatment group compared. 


\section{Figure A3.3: Non-Significant Sex Comparisons}

A

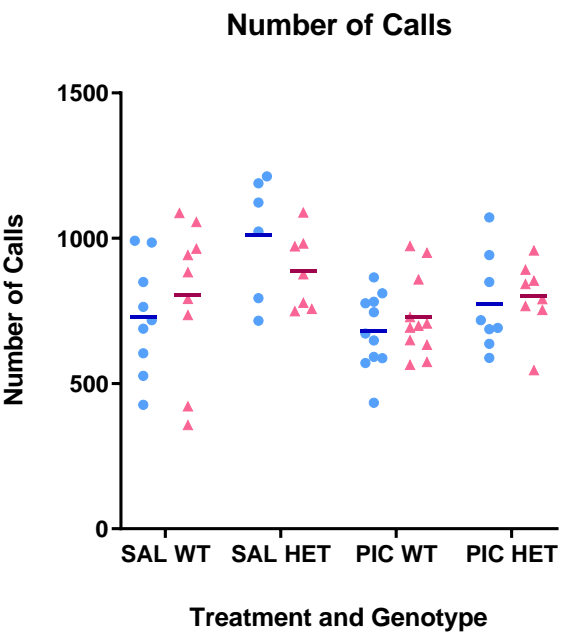

C

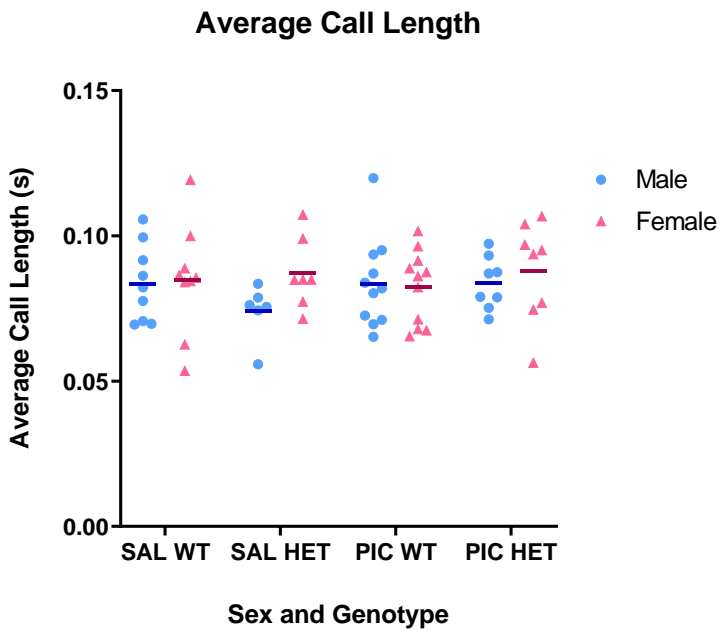

B

Time Calling

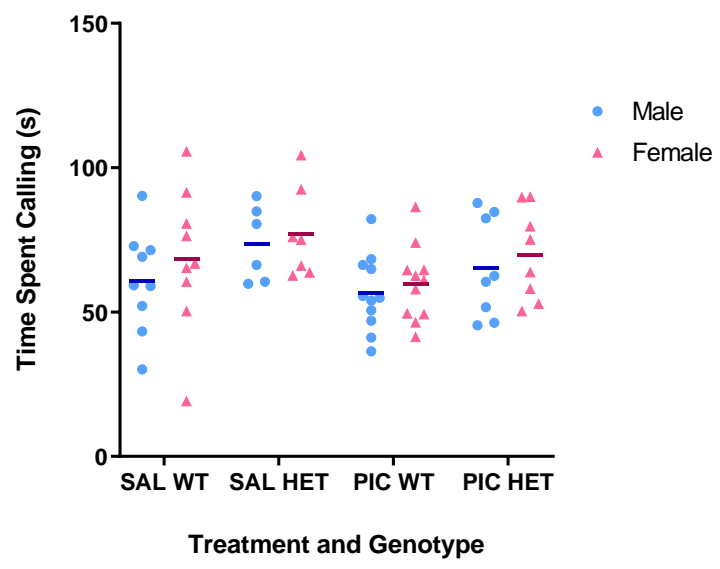

D

Average Principal Frequency

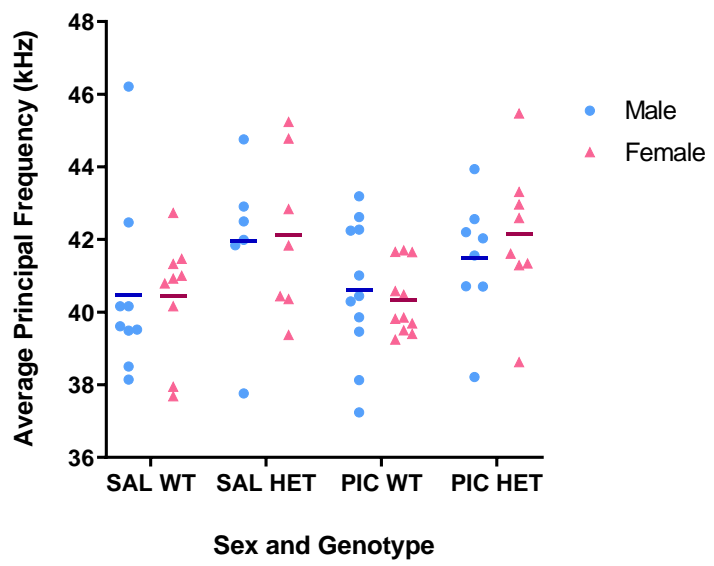

$\mathbf{E}$ Average Frequency Change

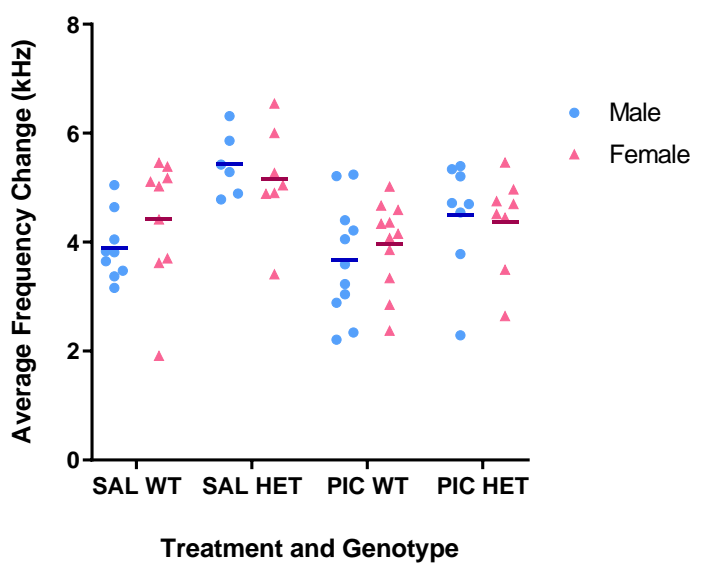

Figure A3.2: Pairwise comparisons between sex in genotype/treatment groups across 5/6 USV metrics did not meet statistical significance. 


\section{Appendix 4: Differentiation of Gene Expression Targets by Sex and Genotype}

Figure A4.1: Gene Expression of Poly I:C and Saline Exposed Pups Grouped by Sex and Genotype

A

Gene Expression in Poly I:C-Exposed Offspring

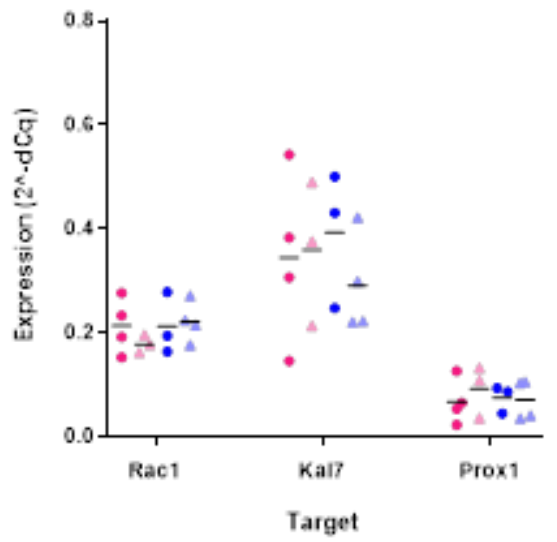

C

Gene Expression in Poly I:C-Exposed Offspring

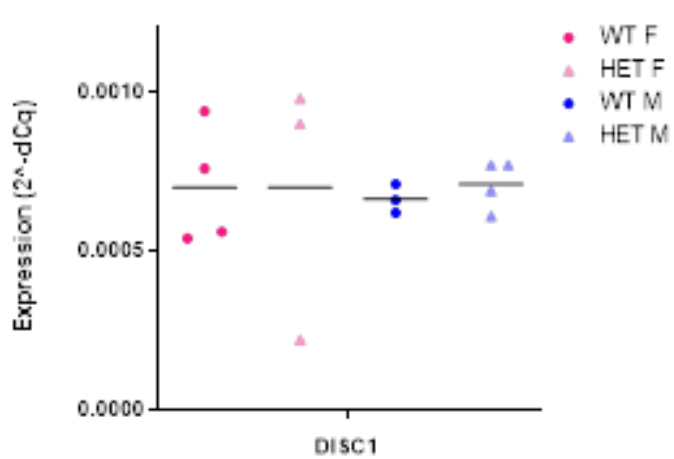

B

Gene Expression in Saline-Exposed Offspring

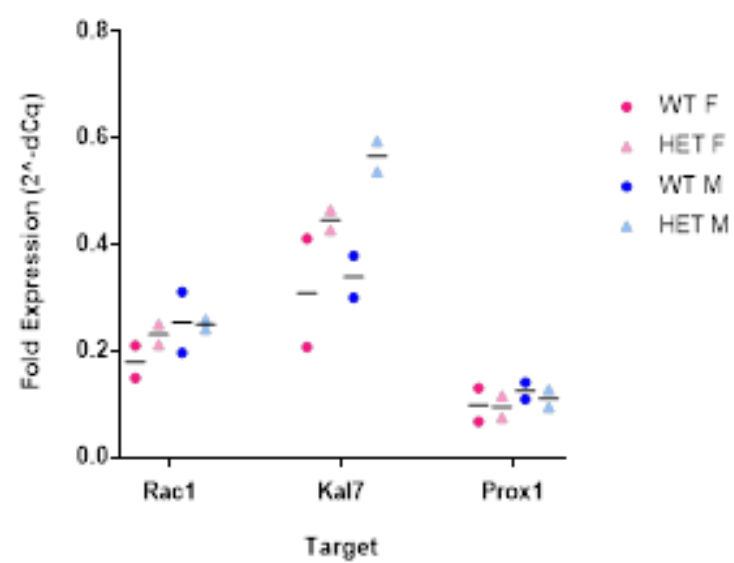

D

Gene Expression in Saline-Exposed Offspring

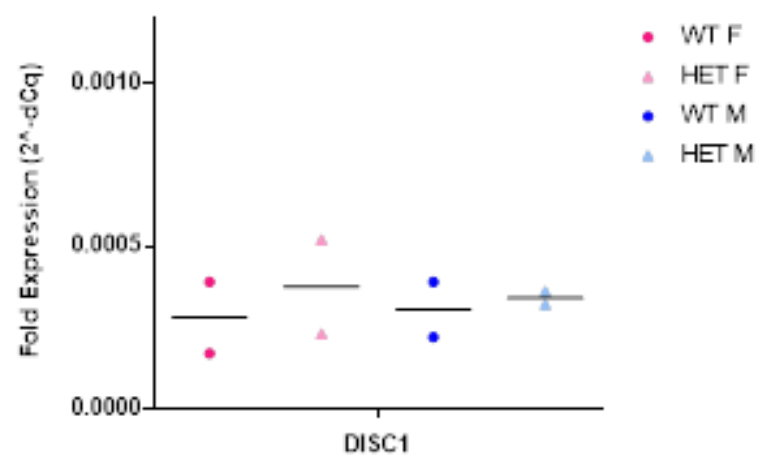

Figure A4.1: Poly I:C and Saline treatment group pup gene expression is differentiated into sex/genotype groups, to view trends within treatment effect. Statistical analysis has not been applied to these figures due to low sample size within sex/genotype groups, which is not sufficient to have confidence in the results. Visual trends indicate that no sex or genotype effect is present in Disc1 expression (C-D), while potential sex differences may be present in Rac1 expression and genotype differences may be present in Kal7 for saline group pups (B). 